BBA 86127

\title{
Electron transfers in chemistry and biology
}

\author{
R.A. Marcus ${ }^{\text {a }}$ and Norman Sutin ${ }^{b}$ \\ a Arthur Amos Noyes Laboratory of Chemical Physics, California Institute of Technology, Pasadena, CA 91125, and \\ ' Department of Chemistry, Brookhaven National Laboratory, Upton, NY 11973 (U.S.A.)
}

(Received October 1st, 1984)

\section{Contents}

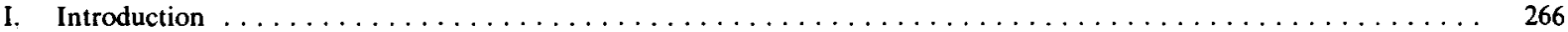

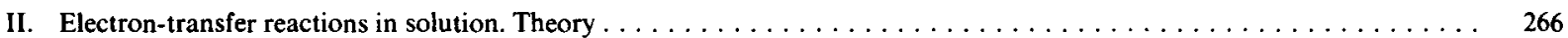

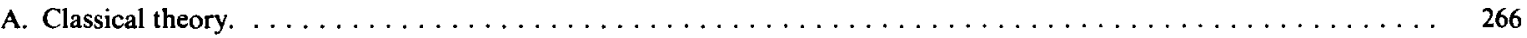

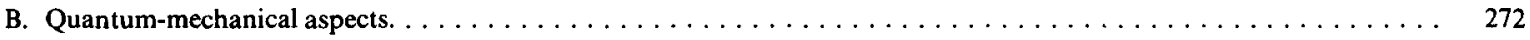

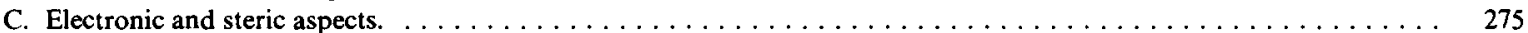

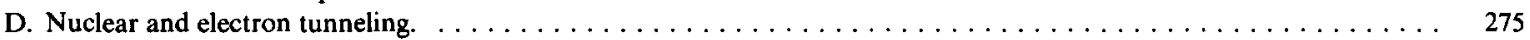

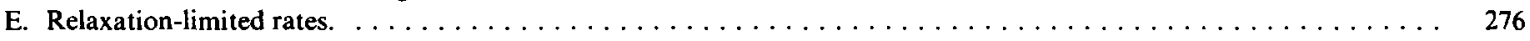

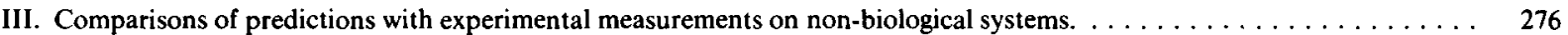

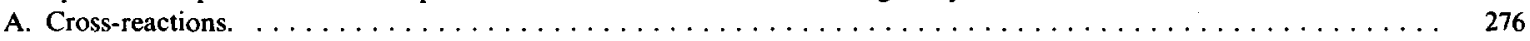

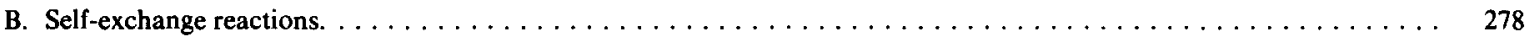

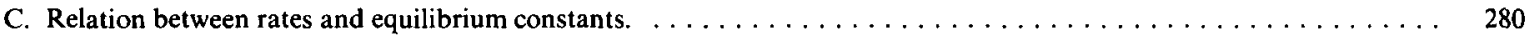

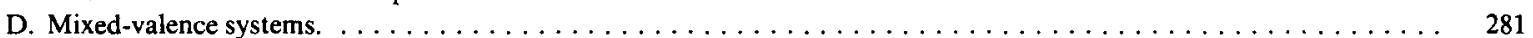

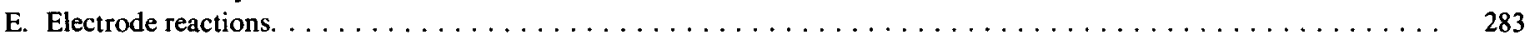

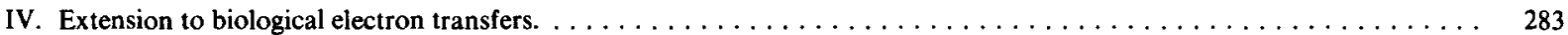

A. Similarities and differences with small molecule electron transfers. $\ldots \ldots \ldots \ldots \ldots$

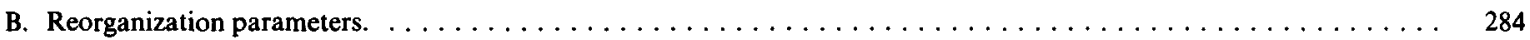

C. Electron tunneling barriers. Distance dependence of electron-transfer rate in nonbiological systems. $\ldots \ldots \ldots 286$

D. Work terms for bimolecular reactions involving metalloproteins. $\ldots \ldots \ldots \ldots \ldots \ldots$

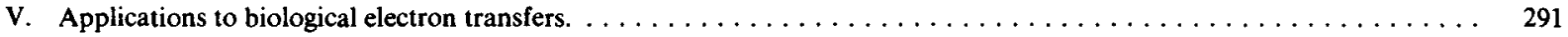

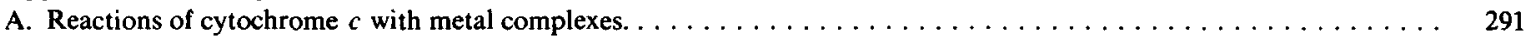

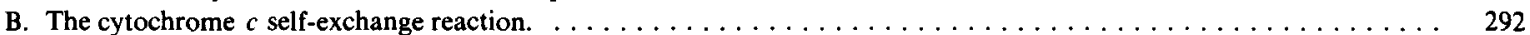

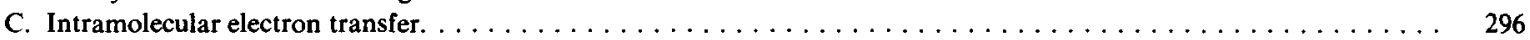

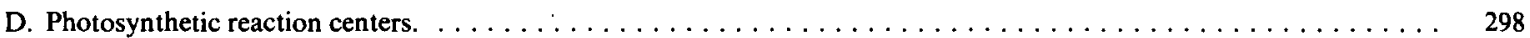

E. Reactions of cytochrome $c$ with cytochrome $b_{5}$ and cytochrome $c$ oxidase. $\ldots \ldots \ldots 301$

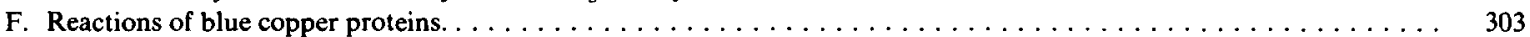

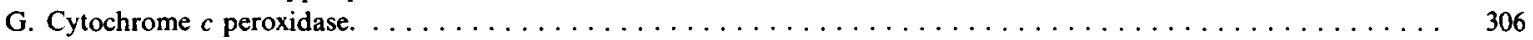

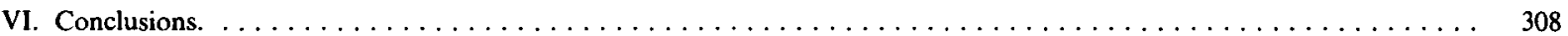

Appendix I. Expressions for activation- and diffusion-controlled reaction rates. $\ldots \ldots \ldots \ldots$

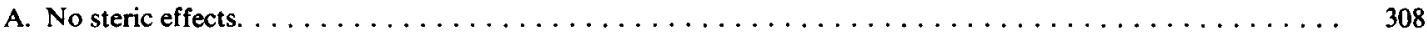

Abbreviations: Cyt $c$, cytochrome $c$; cp, cyclopentadienyl radical; hfac, hexafluoroacetylacetonate ion; bpy, 2,2'-bipyridine; phen, 1,10-phenanthroline, ( $F C$ ), Franck-Condon factor; WKB,

Wentzel-Kramers-Brillouin; $\mathrm{CcP}$, cytochrome $c$ peroxidase; EXAFS, extended X-ray-absorption fine structure. 
B. Steric effects included. $\ldots \ldots \ldots \ldots \ldots \ldots \ldots \ldots \ldots \ldots \ldots \ldots \ldots \ldots \ldots$

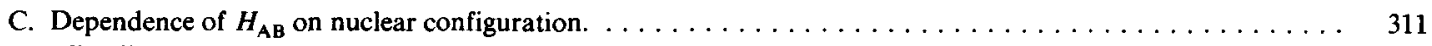

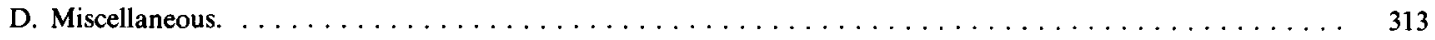

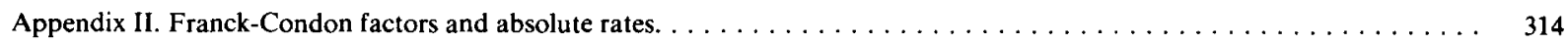

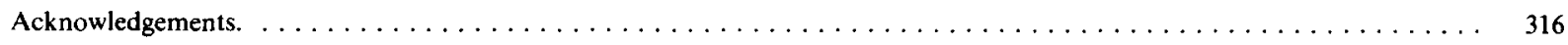

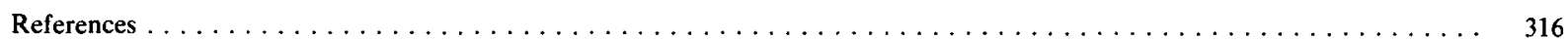

\section{Introduction}

Electron-transfer reactions between ions and molecules in solution have been the subject of considerable experimental study during the past three decades. Experimental results have also been obtained on related phenomena, such as reactions between ions or molecules and electrodes, chargetransfer spectra, photoelectric emission spectra of ionic solutions, chemiluminescent electron transfers, electron transfer through frozen media, and electron transfer through thin hydrocarbon-like films on electrodes.

For 'outer-sphere' electron-transfer reactions in solution there have also been extensive developments in the theory. Quantitative predictions of the theory, without adjustable parameters, include [1-3] (1) rate constants of 'cross-reactions' from those of self-exchange reactions, (2) the relation between the rate constant of a reaction and the driving force for the reaction (standard free energy of reaction $\Delta G^{0}$ ), (3) nonspecific solvent effects on the rate constant, (4) the relation between rates of self-exchange reactions in solution and rate of electron transfer between one of the reactants and an electrode, (5) the calculation of rate constants of electron-transfer reactions from molecular size, bond length changes, vibration frequencies, and solvent properties, and (6) the 'inverted' effect.

By and large, as shown later in this review, these predictions have been successful for a large body of experimental data. In some cases, cited later, there have been major deviations, which have sometimes been used as diagnostic mechanistic tools. In some cases, too, there have been trends in relatively minor discrepancies and these, too, are discussed.

Some of these theoretical predictions (notably those arising from the dependence of the activa- tion-free energy barrier on 'intrinsic' $(\lambda)$ and thermodynamic ( $\left.\Delta G^{0}\right)$ factors) have been extended to other quite different types of reactions, such as atom and proton transfers [4-6], group transfers [7,9], hydride transfers [10,11], proton-bound dimers [12], gas phase ion-molecule reactions [13,14] and conformational equilibria [15]. The main questions, however, for the present article, are: (1) what predictions for reactions in solution can be extended to electron transfers in biological systems, and (2) what can the theory say about the interpretation of some current experimental results? Both of these questions will be addressed.

We begin with a review of the concepts and equations for electron transfers in solution.

\section{Electron-transfer reactions in solution. Theory}

\section{IIA. Classical theory}

The theory of electron-transfer reactions in solution has been summarized and reviewed in many texts and articles, e.g., Refs. 16-27. In the present section we briefly summarize the relevant concepts and equations, so as to serve as a basis for the comparison with experiment and for their extension to biological systems.

We shall be concerned in this section primarily with what has been termed outer-sphere bimolecular electron-transfer reactions, i.e., with reactions in which the two reactants do not share a common atom or group, or, more generally, reactions in which the interaction of the relevant electronic orbitals of the two centers is weak. This circumstance greatly simplifies the theoretical development.

For reaction to occur, the reactants must approach each other to enhance the coupling of their electronic orbitals, and at the same time there 
must be fluctuations in other coordinates. As in any reaction the system as a whole proceeds from configurations (coordinates) in the vicinity of the equilibrium ones for the reactants to those for the products. In the present case, these coordinates involve vibrational coordinates of the reactants, when their equilibrium values differ before and after electron transfer, as well as the orientational coordinates of the surrounding solvent molecules: averaged equilibrium orientations of those solvent molecules differ for reactants and products because each reactant undergoes a change of charge.

The potential energy of the reactants and surrounding medium is a function of all of these nuclear coordinates (thousands of them), and defines a many-dimensional potential-energy surface. A one-dimensional profile of such a many-dimensional surface is given schematically in Fig. 1, and is labelled $\mathrm{R}$. The profile for the products and surrounding medium is labelled $\mathrm{P}$. If there are $N$ coordinates the intersection point in Fig. 1 represents an $(N-1)$-dimensional surface in the $N$-dimensional coordinate space ".

Because the electron being transferred is such a light particle, the Franck-Condon principle ${ }^{\S}$ can be applied to this weak overlap electron transfer (small overlap of the electronic orbitals). It then follows that transfer will occur only at or near nuclear configurations for which the total potential energy of the reactants and surrounding medium is equal to that of the products and

\footnotetext{
I The actual profile of the many-dimensional surface is more complicated than Fig. 1 when there are solvation contributions - there are many local potential-energy minima, for example. In that case only the profile of the free energy (in effect, a Boltzmann-weighted profile) of a system consisting of the pair of reactants, at a given separation, and their environment, along the reaction coordinate could have a quadratic appearance. Such an approximation is much less drastic than assuming that the potential energy is a quadratic function of the coordinates.

According to the Franck-Condon principle, during the actual (instantaneous) electron transfer the nuclei do not have time to change either their positions or their momenta. To satisfy reasonably both conditions simultaneously, the transfer has to occur at nuclear configurations stated in this paragraph, namely at or near the intersection region in Fig. 1. The entropy of the reactants (plus environment) and that of the products (plus environment) are equal on the intersection surface, as are their energies and hence, also, their free energies [2].
}

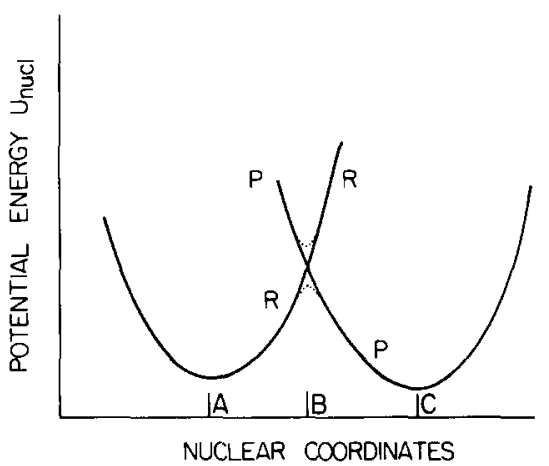

Fig. 1. Profile of many-dimensional potential surface of reactants plus surrounding medium $(R)$ and that of products plus medium ( $P$ ). Dotted lines indicate splitting due to electronic interaction of reactants. $\mathrm{A}$ and $\mathrm{C}$ denote nuclear coordinates for an equilibrium configuration of reactants and for products, respectively; $B$ denotes nuclear configuration at the intersection of the two potential-energy surfaces.

surrounding medium, i.e., at the intersection in Fig. 1. Thermal fluctuations of the coordinates from values near the minimum of the $R$ surface are needed in order for the reacting system to reach the intersection region in Fig. 1.

Fluctuations in the vibrational coordinates need be considered when a reactant has different equilibrium bond lengths (or bond angles) in its two redox states. The systems studied vary widely in this respect, as we shall see in Section III. In addition, fluctuations in the orientational coordinates of the solvent molecules are needed when the solvent is polar. In the latter case the mean orientation of each solvent molecule at any position near the reacting pair is significantly different for reactants and products, as already noted.

Once the system reaches the intersection of the two curves in Fig. 1 the probability of going from the $R$ to the $P$ surface depends on a number of factors, such as the extent of coupling of the electronic orbitals of the two reactants, which in turn depends on the separation distance $r$ of the reactants or, more precisely, on the dominant range of separation distances contributing to the reaction (cf. discussion later).

It is useful at this point to consider the effective potential energy curve for the electronic motion, and relate it to plots such as the one in Fig. 1 for the nuclear motion. A series of such curves for 

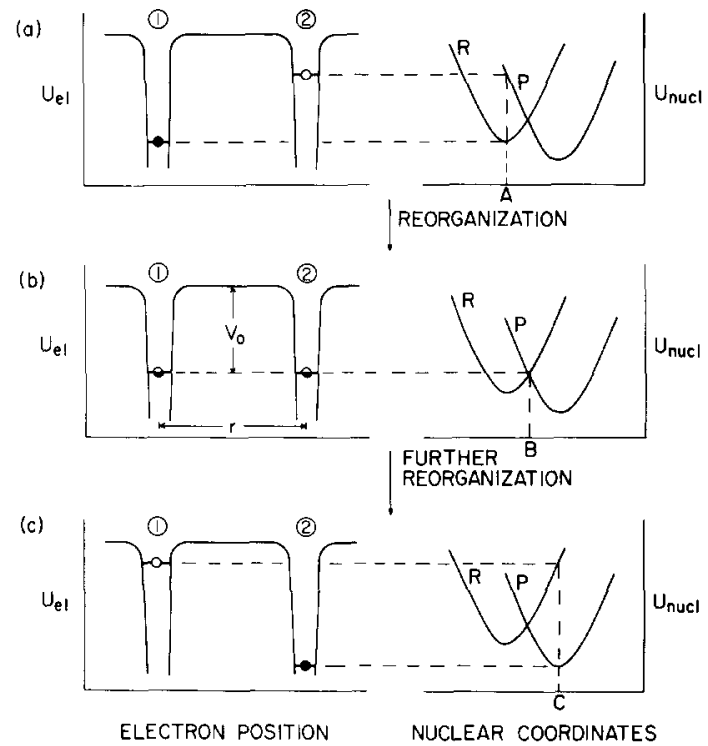

Fig. 2. Electronic potential-energy curves (on left) and the corresponding (nuclear) potential-energy curves on the right. The electronic energies in the two wells are indicated on the left for three nuclear configurations $\mathrm{A}, \mathrm{B}$, and $\mathrm{C}$ for the reaction $\operatorname{red}_{1}+\mathrm{ox}_{2} \rightarrow \mathrm{ox}_{1}+\mathrm{red}_{2}$. The levels in the wells are vertical ionization energies: the filled and open circles denote, respectively, ionization of the reduced state at its equilibrium nuclear configuration and at the equilibrium configuration appropriate to its oxidized state; the half-filled circles refer to ionization of the reduced state at the nuclear configuration appropriate to the intersection region. The level in well 1 is initially occupied in (a) and the level in well 2 in (c).

different nuclear configurations $(\mathrm{A}, \mathrm{B}$, and $\mathrm{C}$ in Fig. 1) is shown in Fig. 2a-c. The effective one-electron potential-energy curve with two wells is given in Fig. 2a. Also given are two electronicenergy levels of the entire system, one when an electronic orbital of reactant 1 is occupied, and the other when an orbital of reactant 2 is occupied. Initially, in a reaction between the reduced form of reactant 1 and the oxidized form of reactant 2 , an electronic orbital of reactant 1 is occupied. The nuclear configuration is labelled $\mathrm{A}$ in Fig. 1 (and in Fig. 2a), and is a configuration that is an equilibrium one for the reactants (' $\mathrm{red}_{1}$ ' and ' $o \mathrm{x}_{2}$ '). The relation of these two electronic-energy levels in Fig. 1 to the nuclear potential-energy curves is indicated by the dashed lines in Fig. 2a.

After a suitable fluctuation of positions of the nuclei in the system prior to electron transfer, a nuclear configuration such as B in Fig. 1 is formed and the positions of the two electronic-energy levels are indicated on the left-side of Fig. $2 b$; that of the electronic orbital in well 1 has been raised and that of well 2 has been lowered. The relationship of these levels to Fig. 1 is again indicated by dashed lines in Fig. $2 b$. Now the electron can transfer from well 1 to well 2 .

A further fluctuation in the positions of the nuclei in the entire system to form a set of nuclear configurations which is an equilibrium one for the products leads to Fig. 2c. The electronic energy when well 2 is occupied now is the lower one. The relation of these energy levels to Fig. 1 is again indicated by dashed lines in Fig. 2c.

We shall denote by $\kappa(r)$ the transmission coefficient or averaged transition probability for electron transfer per passage of the system through the intersection region of Fig. 1, at a fixed separation distance $r$ of the two reactants. This $\kappa(r)$, discussed in Section IV, is usually assumed to vary at large $r$ approximately as $\exp (-\beta r)$ ". When suitably averaged over $r, \kappa(r)$ will be denoted by $\kappa$. For a reaction in which there is substantial electronic coupling between the reactants ('adiabatic reactions'), $\kappa$ is about unity [25].

The calculation of the rate constant of an electron-transfer reaction involves calculating the probability of reaching the many-dimensional intersection region, due to the fluctuations described above, multiplied by a suitably weighted frequency for crossing the intersection region and by a transition probability for going from the $R$ to the $P$ surface. The net result of this calculation is that the rate constant $k$ of a bimolecular electrontransfer reaction is given by

$k=\kappa A \sigma^{2} \exp \left(-\Delta G^{*} / R T\right)$,

where $A \sigma^{2}$ has dimensions of collision frequency (its value is given in Appendix I), $\sigma$ is the average center-to-center distance in the reacting pair during the electron transfer (the mean separation distance in the transition state of the reaction), $\Delta G^{*}$ is the free energy of activation that is related to $\lambda$ (described below), to $\Delta G^{0}$, the 'standard' free en-

\footnotetext{
T Evidence that in some systems the distance dependence may be somewhat less than exponential is given in Ref. [28].
} 
ergy of reaction in the prevailing medium ", and to the work of bringing the reactants $\left(w^{\mathrm{r}}\right)$ or products $\left(w^{\mathrm{p}}\right)$ to the mean separation distance $\sigma[3,27]$ :

$\Delta G^{*}=w^{\mathrm{r}}+\frac{\lambda}{4}\left(1+\Delta G^{0 \prime} / \lambda\right)^{2}$

$\Delta G^{0 \prime}=\Delta G^{0}+w^{\mathrm{p}}-w^{\mathrm{r}}$

Molecular theory (specifically the use of molecular vibrational coordinates) was used to calculate the contribution $\lambda_{i}$ of changes in the bond lengths of the reactants to $\lambda$. To obtain a simple expression for the contribution $\lambda_{\mathrm{o}}$ of changes in solvent orientation coordinates to $\lambda$, dielectric continuum theory was used. (A statistical mechanical treatment is also given in Ref. 3.) In this way $\lambda$ was found to be given by $[3,27]$ :

$\lambda=\lambda_{\mathrm{i}}+\lambda_{\mathrm{o}}$

$\lambda_{i}=\Sigma_{j} \frac{f_{j}^{\mathrm{r}} f_{j}^{\mathrm{p}}}{f_{j}^{\mathrm{r}}+f_{j}^{\mathrm{p}}}\left(\Delta q_{j}\right)^{2}$

$\lambda_{\mathrm{o}}=(\Delta e)^{2}\left[\frac{1}{2 a_{1}}+\frac{1}{2 a_{2}}-\frac{1}{r}\right]\left[\frac{1}{D_{\mathrm{op}}}-\frac{1}{D_{\mathrm{s}}}\right]$

where $f_{j}^{r}$ is the $j$ th normal mode force constant in the reactants. $f_{j}^{\mathrm{p}}$ is that in the products, $\Delta q_{j}$ is the change in equilibrium value of the $j$ th normal coordinate, $\Delta e$ is the charge transferred from one reactant to the other, $a_{1}$ and $a_{2}$ are the radii of the two (spherical) reactants, $r$ is the center-tocenter separation distance ( $r$ equals the $\sigma$ in Eqn. 1 ), and $D_{\mathrm{s}}$ and $D_{\mathrm{op}}$ are the static and optical (square of refractive index) dielectric constants of the solvent, respectively.

In the derivation of Eqns. 1-6, the vibrations

T The $\Delta G^{0}$ introduced here is obtained from concentration measurements in the prevailing medium and in Refs. 3 and 27 is shown to be the appropriate quantity to be used in Eqns. 1-3. It is slightly different from the standard free energy of reaction which is based on standard states. The present $\Delta G^{0}$ was denoted by a primed quantity in Refs. 3 and 27 but we use a simpler notation here and use a primed quantity for the work-corrected free energy of reaction, defined in Eqn. 3. The $\Delta G^{0}$ in Eqn. 3 equals the free energy of reaction when the reactants are a distance $r$ apart in the prevailing medium. within the reactants were treated as harmonic oscillators, but no such restrictive assumption was made for the solvent outside of the coordination shells. What was assumed was, rather, that the dielectric polarization outside the coordination shells responded linearly to any change in (real or fictitious) charges, and hence that free energy depended quadratically on charging parameters ('dielectric unsaturation approximation') $[1-3]^{\S}$. Such a treatment allows the individual solvent dipoles to move very anharmonically, as indeed they do in a liquid.

Equations 1-6 can be given a physical interpretation discussed elsewhere [26]. For example, when $D_{\mathrm{s}}$ equals $D_{\mathrm{op}}$, the solvent molecules are nonpolar and $\lambda_{o}$ vanishes. Again, the larger the radii of the reactants, the smaller the charge-solvent interactions and the smaller $\lambda_{\mathrm{o}}$.

The 'cross-relation' $[2,3,27]$ follows from these equations, by noting from Eqns. 4-6 that $\lambda$ for the cross-reaction $\left(\lambda_{12}\right)$, Eqn. 7 ,

$\operatorname{red}_{1}+\mathrm{ox}_{2} \stackrel{k_{12}}{\rightarrow} \mathrm{ox}_{1}+\operatorname{red}_{2}$

is approximately the mean of the $\lambda$ 's of the self-exchange reactions $\lambda_{11}$ and $\lambda_{22}$.

$\mathrm{red}_{1}+\mathrm{ox}_{1} \stackrel{k_{11}}{\rightarrow} \mathrm{ox}_{1}+\mathrm{red}_{1}$

$\mathrm{red}_{2}+\mathrm{ox}_{2} \stackrel{k_{22}}{\rightarrow} \mathrm{ox}_{2}+\mathrm{red}_{2}$

From this result one finds when the transmission coefficients $\kappa$ are approximately unity, and when the self-exchange rate constants $k_{i i}$ are either corrected for the work terms or when the latter approximately cancel, that $k_{12}$ is given by Eqn. 10:

$k_{12}=\left(k_{11} k_{22} K_{12} f_{12}\right)^{1 / 2}$

\footnotetext{
$\$$ As indicated earlier, such an assumption about the free energy, a thermodynamic quantity which involves statistical averaging over many coordinates, is much less drastic than assuming that the potential-energy function for the surrounding solvent molecules is a quadratic function of their coordinates.
} 
where $K_{12}$ is the equilibrium constant of reaction 7 in the prevailing medium, i.e., $K_{12}$ is equal to $\exp \left(-\Delta G^{0} / R T\right)$, and $f_{12}$ is a known function of $k_{11}, k_{22}$ and $K_{12}\left(\ln f_{12}=\frac{1}{4}\left(\ln K_{12}\right)^{2} / \ln \left(k_{11} k_{22} /\right.\right.$ $Z^{2}$ ), where $Z=A \sigma_{11} \sigma_{22}$; in practice $f_{12}$ is often close to unity). A modification of this equation when the $\kappa$ 's are less than unity has been given [21].

Several applications of Eqns. 1-6 are given in Section III, including comparison of the cross-relation Eqn. 10 with the experimental data, ab initio calculation of rate constants, study of nonspecific solvent effects, and relation between rates and equilibrium constants.

We have already noted that a range of separation distances $r$ contributes to the rate constant. To make $\kappa(r)$ large, $r$ should be small; on the other hand, any short-range or Coulombic repulsion between the reactants will favor large $r$. A compromise in $r$ results, and we obtain approximately Eqn. 1 (cf. Ref. 3 and Appendix I). More refined rate expressions can also be derived.

Expressions have also been derived for the outer-sphere electron transfer between an ion or molecule and an electrode [27,29-31]. The expressions are similar to Eqns. (1-6), only now $A \sigma^{2}$ is replaced by a related quantity which has dimensions of a collision frequency of the ion with unit area of the electrode $\left(\approx 1 \cdot 10^{4} \mathrm{~cm} \cdot \mathrm{s}^{-1}\right), r$ is now twice the distance of the center of the ion to the electrode surface, $1 / 2 a_{2}$ is missing from Eqn. 6 ( $a_{2}$ tends to infinity), the summation in Eqn. 5 is over the vibrational coordinates of one ion instead of two, and $\Delta G^{0}$ is replaced by the activation overpotential. When the rate constant for self-exchange in solution $k_{\mathrm{ex}}$ is then compared with the rate constant for reaction between one of these ions and an electrode at zero activation overpotential, $k_{\mathrm{el}}$, one finds from Eqns. 1-6 and from the equations for the corresponding reaction at an electrode that, approximately,

$$
\left(\frac{k_{\mathrm{ex}}}{Z_{\mathrm{soln}}}\right)^{1 / 2} \geq \frac{k_{\mathrm{el}}}{Z_{\mathrm{el}}}
$$

where $Z_{\text {soln }}$ denotes $A \sigma^{2}$ for the solution reaction and $Z_{\mathrm{el}}$ the corresponding quantity for the electrode reaction. Equality exists when the averaged center-to-center separation distance $\sigma$ of the reactants in the homogeneous reaction is twice that from the center of the reactant to the electrode. However, an adsorbed solvent layer on the electrode may cause the latter separation distance to be somewhat larger. The effect of this increased $r$ on $k_{\text {el }}$ causes the inequality in Eqn. 11, and the effect can be calculated. The equations are applied to electrode systems in Section III.

In deriving the preceding formulae the motion of the nuclei was treated classically. In practice there may be some nuclear tunneling through the barrier in Fig. 1, e.g., depicted schematically by a horizontal motion from $a$ to $b$ in Fig. 3. The nuclear tunneling in Fig. 3 at room temperature typically accounts, in the so-called normal region, for only a relatively minor factor ( $\Gamma$ in Table II) in the rate constants, which due to variations in $\lambda$ and $\Delta G^{0}$, may vary by some 15 or more orders of magnitude from system to system. Examples are given later in Table II. The cross-relation in the normal region is even less affected, because of partial cancellation of the quantum corrections in the ratio $k_{12} /\left(k_{11} k_{22} K_{12}\right)^{1 / 2}$. Nuclear tunneling in the 'inverted region', defined below, has a larger effect, as discussed later.

When $\Delta G^{0}$ is made increasingly negative at constant $\lambda$, for example, by suitable variation of a ligand in a reactant, one sees from Eqn. 2 that the free-energy barrier to reaction $\Delta G^{*}$ initially decreases and that, when $-\Delta G^{0}$ exceeds $\lambda, \Delta G^{*}$ begins to increase. This region where $-\Delta G^{0 \prime}>\lambda$ has been termed the inverted region [3], and some consequences are described in Section III. Physi-

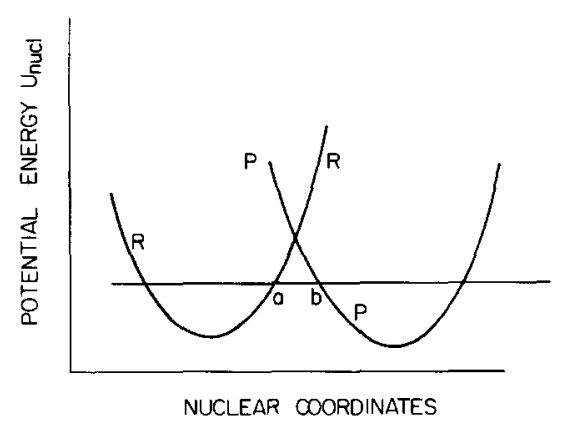

Fig. 3. As in Fig. 1, but describing nuclear tunneling through the barrier from the $R$ to the $P$ surface (from a to $b$ ). 
cally, in terms of Fig. 1, this effect of making $\Delta G^{0}$ more negative at constant $\lambda$ can be seen to correspond to lowering the $P$ surface vertically (or raising the $R$ surface). The intersection of the $R$ and $P$ surfaces eventually occurs at the minimum of the $R$ surface and there is then no barrier. Further lowering raises the point of intersection, as in Fig. 4, and hence now raises the barrier, i.e., increases $\Delta G^{*}$.

In the case of Fig. 4, nuclear tunneling at some energy, from the $R$ to the $P$ surface, is represented by a horizontal motion from a to b, as in Fig. 5 ? Here, the slopes of the potential-energy surfaces $R$ and $P$ have the same sign near the intersection region, unlike the case in Fig. 3, and the semiclassical theory of nuclear tunneling shows that tunneling is now much more important. Typically, nuclear tunneling is treated in the case of Figs. 3 and 5 either by calculation of quantum mechanical Franck-Condon factors (appearing in the next section) ${ }^{\S}$ or by semiclassical nuclear tunneling equations. The theoretical equations for these quantum

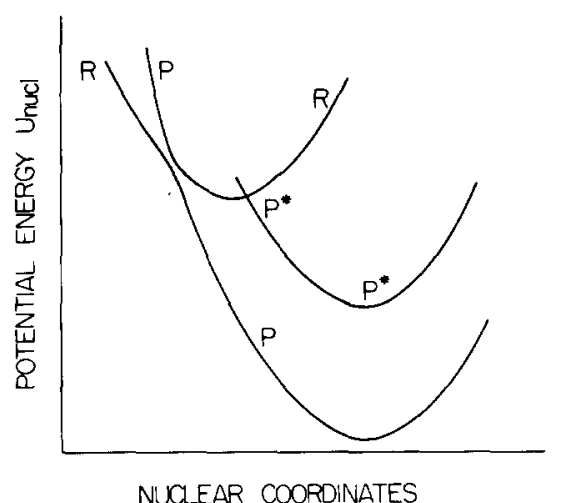

Fig. 4. Highly exothermic reaction to form the electronic ground-state products (surface P) or products where one is electronically excited (surface $\mathrm{P}^{*}$ ).

\footnotetext{
In most chemical reactions nuclear tunneling is usually important only for protons. However, for the present reactions, the potential-energy barrier for changes in metal-ligand bond lengths is so narrow (e.g., as seen from the $\Delta d_{0}$ 's in Table II) that even ligands can tunnel at room temperature (e.g., see $\Gamma$ factors in Table II).

$\$$ A particular Franck-Condon term in the Franck-Condon sum $(F C)$ in Eqn. 16 of Section IIB is an overlap integral of a reactants' and a products' wavefunction for the nuclear
}

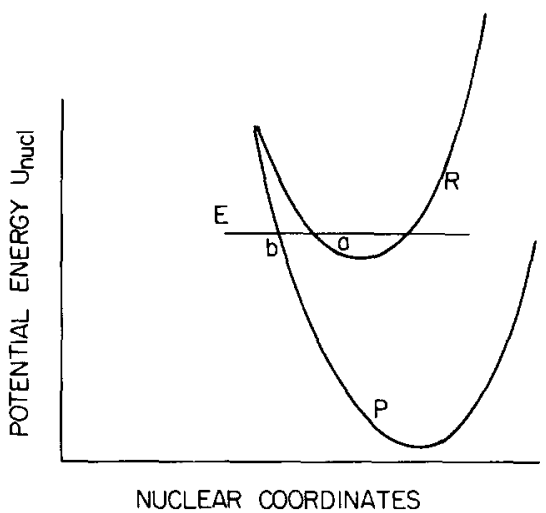

Fig. 5. Nuclear tunneling in a highly exothermic reaction, from a to $b$.

calculations are referred to in Section IIB. Examples of the results are given in Section III.

A corollary of Fig. 4 involves the formation of electronically excited products, i.e., a chemiluminescent reaction occurs: although the ground electronic-state potential-energy surface $P$ for the products may intersect $R$ at a high point, the surface in which one of the products is electronically excited, $\mathrm{P}^{*}$, may intersect in a more favorable region, as in Fig. 4, and then one may obtain preferential formation of the less thermodynamically favored products [33,34]. This preferential $(\approx 100 \%)$ formation of electronically excited products has been observed experimentally [35], and one explanation is the predicted 'inverted effect'. Direct experimental confirmation of the inverted effect has recently been obtained [36-38] (cf. Ref. 39 and also a discussion of other studies [27]).

Various other classical treatments of the problem have also been given, including the treatment

motion. It is small when the region of space occupied by the reactants' relevant wavefunction for the nuclear motion is rather different from that occupied by the products' wavefunction, for example, when there is a large difference of the equilibrium bond lengths and when, at the same time, the two wavefunctions refer to relatively unexcited vibrational quantum states. When the overlap of two such wavefunctions of the same energy is small, it can be shown that an equivalent semiclassical interpretation is that the states are connected not by a classically allowed path but by a nuclear tunneling through a barrier separating the reactants' and products' regions of vibrational coordinate space. (The argument is based on a semiclassical evaluation of the overlap integral, related to that given in Ref. 32.) 
of vibrations and solvation [40] and of vibrations alone [41,42]. The expressions in Ref. 40 for the solvation are the same as those in Ref. 1". The vibrations were treated using calculated force fields rather than experimental vibration frequencies. The vibrational results in Refs. 41 and 42 are for self-exchange reactions, rather than cross-reactions, and a simple force field was assumed (no cross-terms in bond force constants).

In the foregoing discussion we have explicitly considered outer-sphere electron transfers. The latter differ from 'inner-sphere' electron transfers. In the latter, the two reactants approach each other closely enough that they actually share in common a ligand or atom in their coordination shells. This strong interaction is then followed by either an electron, atom, or radical transfer. Such bond formation and rupture requires a modified treatment from that leading to Eqns. 1-6. The cross-relations can still apply $[4,9,10,44]$, provided the cross-reaction and the self-exchange reactions have a similar mechanism.

\section{IIB. Quantum-mechanical aspects}

In Eqns. 1-6 the motion of the nuclei was treated classically. Quantum mechanical treatments of the nuclear coordinates have been made for cases where additional approximations have been imposed (e.g., 45-56). These treatments were almost exclusively for reactions having very weak electronic coupling, namely having $\kappa \ll 1$ in Eqn. 1.

The first-order rate constant for electron transfer at fixed $r$ is now given by

$k=\frac{2 \pi}{\hbar} H_{\mathrm{AB}}^{2}(F C)$,

where $H_{\mathrm{AB}}$ is the electronic matrix element describing the electronic coupling of the reactants' electronic state with the products', and is equal to

\footnotetext{
" Recently, another treatment of the solvent for organic systems has been assumed in which the solvent near a singly charged organic ion is assigned a harmonic oscillator force constant (for the ion-solvent interaction) which is considerably changed when the ion loses its charge [43]. This interpretation is discussed in Appendix ID.
}

one-half the separation of the dotted curves at the intersection of the $\mathrm{R}$ and $\mathrm{P}$ surfaces in Fig. 1. The quantity $(F C)$ is the 'Franck-Condon' factor: it is a sum of products of overlap integrals of the vibrational and solvational wavefunctions of the reactants with those of the products, suitably weighted by Boltzmann factors. Purely vibrational Franck-Condon factors are available in various reviews and articles, e.g., Refs. 21, 45 and 57.

Typically, quantum-mechanical theories [45-56] for the nuclear motion have either:

(i) treated all coordinates (solvent alone or inner-shell plus solvent) at fixed $r$ as a collection of independent oscillators, harmonic or anharmonic ('Morse') $\$$, or

(ii) treated quantum-mechanically the innershell coordinates as harmonic (or Morse) oscillators, but treated the motion (orientational, librational) of the solvent outside the inner-coordination shell as classical, using the free energy of solvent reorganization in Ref. 1.

With this classification in mind we consider next the relation between Eqn. 12 and its classical counterpart for treatment of the motion of the nuclei. When the solvent molecules are treated as a collection of oscillators, namely when assumption (i) is made, Eqn. 12 may be rewritten as

$$
k=\frac{2 \pi}{\hbar} H_{\mathrm{AB}}^{2} \underset{v_{\mathrm{r}}, v_{\mathrm{p}}}{\Sigma}(F C)_{\nu} p\left(v_{\mathrm{r}}\right)
$$

where $(F C)_{v}$ is the Franck-Condon (vibrational overlap) factor for any given set of vibrational quantum numbers $v_{\mathrm{r}}$ of the reactants' and $v_{\mathrm{p}}$ of the products' system (including the solvent oscillators), $p\left(v_{\mathrm{r}}\right)$ is the equilibrium (Boltzmann) probability of finding the system in the vibrational state $v_{\mathrm{r}}$, and the sum is over all $v_{\mathrm{r}}$ and $v_{\mathrm{p}}$. When all of the vibrational frequencies $\nu_{i}$ in $(F C)$ are relatively small, e.g., $h \nu_{\mathrm{i}} / 2 k T \leqslant 1$, and when they are unchanged by reaction, Eqn. 13 reduces to

\footnotetext{
$\$$ With the aid of this assumption, the quantity $(F C)$ in Eqn. 12 can be evaluated. However, when the solvent is treated more realistically, namely, by permitting the solvent molecules to perform their actual complicated coupled anharmonic motions, no practical evaluation of $(F C)$ has yet been made. Only a formal expression involving overlap integrals for the entire system has been given [58].
} 
$k=\frac{2 \pi}{\hbar} H_{\mathrm{AB}}^{2} \frac{1}{(4 \pi \lambda R T)^{1 / 2}} \mathrm{e}^{-\left(\Delta E^{\left.0^{\prime}+\lambda\right)^{2} / 4 \lambda R T}\right.}$

where $\Delta E^{0^{\prime}}$ is the energy (enthalpy) of reaction at the separation distance $r$ in the prevailing medium and is negative for an exothermic reaction. On the other hand, when only some of the vibration frequencies are treated as relatively small and unchanged by reaction, with a contribution to $\lambda$ denoted by $\lambda_{0}$, one obtains $[47,49,50]$ from Eqn. 13:

$$
\begin{aligned}
k= & \frac{2 \pi}{\hbar} H_{\mathrm{AB}}^{2} \sum_{v_{\mathrm{r}}, v_{\mathrm{P}}} \frac{1}{\left(4 \pi \lambda_{\mathrm{o}} R T\right)^{1 / 2}} \\
& \times \mathrm{e}^{-\left(\Delta E^{0}+\Delta \varepsilon_{\mathrm{o}}+\lambda_{\mathrm{o}}\right)^{2} / 4 \lambda_{\mathrm{o}} R T}(F C)_{v} p\left(v_{\mathrm{r}}\right)
\end{aligned}
$$

In applications of Eqn. 15, $\lambda_{0}$ is typically intended to represent the orientational contribution from the solvent molecules outside the inner-coordination shells of the reactants and is usually assumed to be given by Eqn. 6 . The quantity $\Delta \epsilon_{v}$ is the vibrational energy of the products minus that of the reactants for the set of quantum numbers $\left(v_{\mathrm{r}}, v_{\mathrm{p}}\right)$.

When assumption (ii) is made instead, Eqn. 13 can be rewritten $[52,55,56]$ as Eqn. 16, which now contains $\Delta G^{0^{\prime}}$ instead of the $\Delta E^{0^{\prime}}$ in the exponent in Eqn. 15:

$$
\begin{aligned}
k= & \frac{2 \pi}{\hbar} H_{\mathrm{AB}}^{2} \sum_{v_{\mathrm{r}}, v_{\mathrm{P}}} \frac{1}{\left(4 \pi \lambda_{\mathrm{o}} R T\right)^{1 / 2}} \\
& \times \mathrm{e}^{-\left(\Delta G^{0^{\prime}}+\Delta \epsilon_{r}+\lambda_{\mathrm{o}}\right)^{2} / 4 \lambda_{\mathrm{o}} R T}(F C)_{v} p\left(v_{\mathrm{r}}\right)
\end{aligned}
$$

The factor in the sum in Eqn. 16 multiplying $(F C)_{v} p\left(v_{\mathrm{r}}\right)$ is equivalent to a Boltzmann-weighted sum of solvational Franck-Condon factors. Eqn. 16 has been recently derived [58] using semiclassical theory (e.g., Ref. 59) to treat the reorientational motion of the solvent. In that way, the reorientational contribution of the solvent to $(F C)$ in Eqn. 12 was converted to a classical expression without introducing any harmonic oscillator or near-harmonic oscillator approximation for the molecular motion of the solvent. In the high-tem- perature limit, Eqn. 16 reduces to

$k=\frac{2 \pi}{\hbar} H_{\mathrm{AB}}^{2} \frac{1}{(4 \pi \lambda R T)^{1 / 2}} \mathrm{e}^{-\left(\Delta G^{0^{\prime}}+\lambda\right)^{2} / 4 \lambda R T}$

where $\lambda$ is $\lambda_{\mathrm{o}}+\lambda_{\mathrm{i}}$. Eqn. 17 has the same form as Eqns. 1 and $2\left(w^{\mathrm{p}}=w^{\mathrm{r}}=0\right.$ in a fixed site intramolecular reaction), and provides by comparison a value when $\kappa \ll 1$ for the $\kappa \nu$ appearing later in Eqn. 31 for this fixed-site system.

Unlike Eqn. 16, Eqn. 15 has a major defect when there is a significant entropy of reaction $\Delta S^{0}$. In the presence of a polar environment there can be large entropic effects, not reproduced by Eqns. 13-15. These entropic effects result mainly from changes in freedom of the rotations and librations of the solvent molecules. An example of a reaction with a large $\Delta S^{0}$ is $\mathrm{Fe}\left(\mathrm{H}_{2} \mathrm{O}\right)_{6}^{2+}+$ $\mathrm{Ru}(\mathrm{bpy})_{3}^{3+} \rightarrow \mathrm{Fe}\left(\mathrm{H}_{2} \mathrm{O}\right)_{6}^{3+}+\mathrm{Ru}(\mathrm{bpy})_{3}^{2+}$ : for this reaction $\Delta S^{0}$ is $-180 \mathrm{~J} \cdot \mathrm{mol}^{-1} \cdot \mathrm{deg}^{-1}$ (e.g., cited in Ref. 59). Under such conditions assumption (i) becomes grossly inadequate. If, however, there were no solvation outside the inner coordination shell, or if that solvent behaved as a collection of harmonic or anharmonic oscillators of fixed frequencies, one would expect $\Delta S^{0} \approx 0$, and $\Delta E^{0}$ $\left(\approx \Delta G^{0}+T \Delta S^{0}\right) \approx \Delta G^{0}$, and then Eqn. 15 would be satisfactory. Because of this inadequacy of assumption (i) and hence of Eqn. 15, Eqn. 16 has frequently been used in articles to estimate quantum effects, e.g., Refs. 48, 52, 53, 56 and 61. In summary, when a large standard entropy change $\Delta S^{0}$ accompanies a reaction, it influences the reaction rate and Eqns. 14 and 15 do not allow for this possibility, while Eqns. 16 and 17 do. Indeed, when there is a nonvanishing $\Delta S^{0}$, Eqns. 14 and 15 and their counterparts for the reverse reaction rate constant $k_{\text {rev }}$ do not obey the correct equilibrium constant relation, $k / k_{\text {rev }}=$ $\exp \left(-\Delta G^{0^{\prime}} / R T\right)$, but Eqns. 16 and 17 do.

Eqns 12-17 were obtained for reactions with $\kappa \ll 1$, as already noted. However, the main quantum correction in Eqn. 16 to the classical equation, Eqn. 17, proves to be one for nuclear tunneling, and the latter can occur regardless of whether $\boldsymbol{\kappa}$ is small or close to unity. Thus, one quantum correction to Eqn. 1, which has been used for $\kappa \approx 1$, is that given by $[53,55,61]$ 
$k_{\text {quantum }}=k_{\text {classical }}(F C)_{\mathrm{q}} /(F C)_{\mathrm{c}}$

where $(F C)_{\mathrm{q}} /(F C)_{\mathfrak{c}}$ is the ratio of the right-hand sides of Eqns. 16 and 17, where the vibrational motion is treated in a quantum manner but the solvent motion is treated classically. Another way of making the quantum correction for nuclear tunneling is the WKB method referred to later.

The ratio $k_{\text {quantum }} / k_{\text {classical }}$ has been calculated for a number of reactions using Eqn. 18: when $\lambda_{\mathbf{i}}$ is relatively small, as in the $\mathrm{Ru}\left(\mathrm{NH}_{3}\right)_{6}^{3+/ 2+}$ selfexchange reaction, the calculated ratio of rate constants is close to unity at room temperature (it equals 1.2) [53]. At relatively large values of $\lambda_{i}$ and appreciable vibration frequencies, for example, for the $\mathrm{Fe}\left(\mathrm{H}_{2} \mathrm{O}\right)_{6}^{3+/ 2+}$ self-exchange reaction, (the metal-ligand symmetric stretching frequency for $\mathrm{Fe}\left(\mathrm{H}_{2} \mathrm{O}\right)_{6}^{3+}$ and for $\mathrm{Fe}\left(\mathrm{H}_{2} \mathrm{O}\right)_{6}^{2+}$ is 490 and 389 $\mathrm{cm}^{-1}$, respectively), the ratio is about 4 [53], and for the $\mathrm{Co}\left(\mathrm{NH}_{3}\right)_{6}^{3+/ 2+}$ self-exchange it is about 6 [53,62]. The ratio of quantum-mechanical rate constants, $k_{12} /\left(k_{11} k_{22} K_{12} f_{12}\right)^{1 / 2}$, for the reaction $\mathrm{Fe}\left(\mathrm{H}_{2} \mathrm{O}\right)_{6}^{2+}+\mathrm{Ru}(\mathrm{bpy})_{3}^{3+} \rightarrow \mathrm{Fe}\left(\mathrm{H}_{2} \mathrm{O}\right)_{6}^{3+}+$

$\mathrm{Ru}(\mathrm{bpy})_{3}^{2+}$ was calculated to differ by only $6 \%$ from its classical value of unity [53]. I.e., these quantum effects have relatively little effect on the cross-relation in such cases. Where quantum effects on the nuclear motion can be quite large is in the inverted region or at low temperature: Refs. 48-52 and 61 present calculations for a number of examples.

In the case of a highly exothermic reaction and when a single vibration frequency $\nu$ is principally involved in the readjustment of nuclear coordinates associated with the electron transfer, the double sum in Eqn. 16 can be approximated by [27]:

$k=\frac{2 \pi}{\hbar} H_{\mathrm{AB}}^{2} \frac{\mathrm{e}^{-S} S^{v} e^{-\left(\Delta G^{0^{\prime}}+v h \nu+\lambda_{\mathrm{o}}\right)^{2} / 4 \lambda_{\mathrm{o}} R T}}{h \nu \Gamma(v+1)}$

where $\Gamma$ is the Gamma function, $S$ is $\lambda_{\mathrm{i}} / h \nu, \Delta \epsilon_{v}$ in Eqn. 15 has become $v h \nu$, and $v$ is approximately the solution of

$\Delta G^{0^{\prime}}+v h \nu+\lambda_{\mathrm{o}}=-\left(\frac{2 \lambda_{\mathrm{o}} R T}{h \nu}\right) \ln \frac{-\left(\Delta G^{0}+\lambda_{\mathrm{o}}\right)}{h \nu}$
Equations 19 and 20 were in good agreement with the quantum result where tested $\left(h \nu=1350 \mathrm{~cm}^{-1}\right.$, and $\lambda_{o}$ not too small, varying from 500 to 2500 $\mathrm{cm}^{-1}$ ) [27]. However, it is reasonably straightforward instead to evaluate the sum in Eqn. 16 numerically or by a 'saddle-point' approximation (e.g., Ref. 53), particularly for a single frequency model. Another approximate quantum expression is given and used in Appendix II.

An alternative treatment has been given for vibrational quantum effects, also known sometimes as the 'semiclassical' treatment $[63,64]$. The expression for the rate constant for electron transfer between fixed sites is of a form rather similar to Eqn. 14, namely:

$k=\frac{2 \pi}{\hbar} H_{\mathrm{AB}}^{2} \frac{1}{(4 \pi \lambda R T \chi)^{1 / 2}} \mathrm{e}^{-\left(\Delta E^{0}+\lambda\right)^{2} / 4 \lambda R T \chi}$

where

$\chi=\frac{h \nu}{2 R T} \operatorname{coth} \frac{h \nu}{2 R T}$

The factor $\chi$ approaches unity when $h \nu / 2 R T$ becomes small. Eqn. 21 is readily extended to the case of many vibrational coordinates and has the advantage of simplicity over Eqn. 12. Its disadvantage is one of accuracy [51,65]. For example for the $\mathrm{Fe}\left(\mathrm{H}_{2} \mathrm{O}\right)_{6}^{3+/ 2+}$ system, Eqn. 21 has an error of a factor of $15-20$ at room temperature [65]. The source of its difficulty has been identified [65], namely in its treatment of nuclear tunneling through the potential-energy barrier in Fig. 1. Only when the products' surface $\mathbf{P}$ is extremely steep at the intersection does this particular error become small [65].

Another treatment, quite different from the above one but also labelled semiclassical, involves use of a semiclassical ('WKB') approximation for the nuclear tunneling probability [66-68]. This treatment has been reviewed recently [21] and examples of its application are given in Ref. [69], both for the cases of small and large $\kappa(\kappa \approx 1)$ in Eqn. 1. It has been developed mainly for singlefrequency systems and, more approximately, for other systems. A more general version (many-dimensional) of semiclassical (WKB) theory was used, as already noted, in deriving Eqn. 16 [58]. 
The main quantum correction for the nuclear motion contained in the $(F C)_{v}$ factor in Eqn. 13, but absent in Eqn. 14 is, as previously noted, one for nuclear tunneling. The semiclassical (WKB) treatment provides one way of estimating this correction.

\section{IIC. Electronic and steric aspects}

The rate of electron transfer depends on the nature of the intervening medium and transfers through a series of conjugated bonds ( $\pi$ bonds) and through $\sigma$ bonds have been discussed $[28,70,71]$. In extended Hückel calculations the dependence of $\kappa$ on $r$ was calculated $[28,70]$ to be larger for $\sigma$ - than for $\pi$-bonded systems. In the present article (Section IV) we shall use some averaged distance dependence for the protein medium; this dependence is suggested by other sources of experimental data (subsection IVC).

Recently, orientation effects on electron-transfer rates have been treated using a simplified cavity model for the the reactants (Ref. 72; Cave, R.J., Klippenstein, S.J., Marcus, R.A., unpublished data, and Cave, R.J., Siders, P. and Marcus, R.A., unpublished data). Orientation effects for $\pi$-electron systems are expected both because of the nonspherical nature of the electronic orbitals and because of the nonspherical shape of the molecules themselves. The relative contributions of the two effects have been investigated by making a calculation (Ref. 72 and Cave, R.J., Klippenstein, S.J. and Marcus, R.A., unpublished data) for spheroids, which includes both effects, and for spheres, which includes only the orbital shape effect. Applications are also being made to $\pi$-electron systems of biochemical interest (Cave, R.J., Siders, P. and Marcus, R.A., unpublished data). One can expect molecules such as the heme part of cytochrome $c$ or the porphyrin part of bacteriochlorophyll to exhibit significant orientation contributions to $H_{\mathrm{AB}}^{2}$.

Steric effects in reactions can also occur, as is evident in the difference between the measured self-exchange rates of optically active d-helicene with its d-helicene anion, as compared with that for the reaction with the l-helicene anion [75]. Steric effects have been calculated [76] for the $\mathrm{Fe}\left(\mathrm{H}_{2} \mathrm{O}\right)_{6}^{3+/ 2+}$ exchange: in this system the steric effect originated from the (calculated) interpenetration of the $\mathrm{H}_{2} \mathrm{O}$ ligands, to produce a greater $H_{\mathrm{AB}}$ at small $r$ (approx. $0.525 \mathrm{~nm}$ ) [77].

\section{IID. Nuclear and electron tunneling}

In this section we distinguish and discuss further nuclear and electron tunneling. Nuclear tunneling was depicted in Figs. 3 and 5 ?. The nuclear tunneling tends to occur at low temperatures, because then the probability is small that the reacting system has enough energy to surmount the barrier in Figs. 3 and 5. At sufficiently low temperatures, in fact, all of the reaction occurs by nuclear tunneling. The rate is then independent of temperature, since reaction is now occurring largely at an energy equal to that of the zero-point vibrational energy of the pair of reactants and surrounding system (provided the energy corresponding to the lowest point of the $R$ surface equals or exceeds that for the $P$ surface).

Electron tunneling occurs when the potential energy acting on the electron in the space between the reacting pair is higher than the energy of the electron in its initial localized site, as in Fig. 2. The nuclear rearrangement permits, as in Fig. $2 b$, the initial and final localized energy levels of the electron to be the same, and the electron can then tunnel through its barrier from one site to the other. This need for equality or near-equality of the electronic-energy levels is a consequence of the Franck-Condon principle and energy conservation: when the electronic energies are the same at the given nuclear configuration (Fig. 2b) then the total energy is conserved, since (using classical terminology and the F-C principle) the momenta of the nuclei and hence their kinetic energies do not change instantaneously during the electron transfer. Although electron tunneling is a convenient term for describing the process of electron transfer in Fig. $2 b$, it is, at best, only a physically intuitive one-electron description of the process. A more fundamental but more abstract approach involves the many-electron quantum mechanical

\footnotetext{
"Recalling that these figures are profiles of many-dimensional surfaces, one sees that this tunneling is also a many-dimensional one.
} 
calculation of the $H_{\mathrm{AB}}$ in Eqns. 12-17.

Temperature affects the nuclear motion and thereby the Boltzmann probability of attaining any range of nuclear configurations such as near point $\mathrm{a}$ in Fig. 3 or near the intersection region in the same figure. Thus it affects the relative contribution of nuclear tunneling to the reaction rate. Temperature does not affect directly the value of $H_{\mathrm{AB}}$, although $H_{\mathrm{AB}}$ will vary somewhat with nuclear configuration (a point neglected in Eqn. 12, where only the dependence of $H_{\mathrm{AB}}$ on the separation distance $r$ is considered; the dependence of $H_{\mathrm{AB}}$ on nuclear configuration is discussed in Appendix IC). Thus, temperature does not affect the electron tunneling in Fig. 2 per se: the existence of a temperature-independent rate constant at low temperatures and a temperature-dependent one at higher temperatures [18] is, instead, a manifestation of nuclear tunneling.

Terms frequently used in the context of electron-transfer reactions are 'adiabatic' and 'nonadiabatic'. In an adiabatic electron transfer there is substantial electronic coupling of the reactant pair: there is enough splitting $2 H_{\mathrm{AB}}$ of the dotted curves in Fig. 1 that this pair remains throughout largely or completely on the lowest potential-energy surface [25]. (In Fig. 1 this lowest surface is the one labelled $R$ in the initial stages of reaction, then is the lower of the two dotted surfaces in the intersection region, and finally becomes the surface labelled $P$ after the passage through that intersection region.) For an adiabatic reaction the $\kappa$ in Eqn. 1 is unity, or approximately so.

In a nonadiabatic reaction the electronic coupling of the reacting pair is so weak, i.e., the $H_{\mathrm{AB}}$ in Eqn. 12 is so small, that the probability $\kappa$ of proceeding from the $R$ to the $P$ surface when the system is in the vicinity of the intersection region in Fig. 1 is very small. Most systems reaching that intersection region now remain on the $R$ surface, instead of going to the P surface. Eqns. 12-17 and 21 assume the reaction to be nonadiabatic. For reactions intermediate between adiabatic and nonadiabatic, and hence with intermediate $\kappa$, expressions of the Landau-Zener type are available for calculating $\kappa$, e.g., cited in Refs. 25 and 67, but the latter is mainly a refinement only infrequently used. In the 'inverted region', namely in the case of Fig. 4, where the solid curves again denote the adiabatic surfaces, a reaction in which the system goes directly from the $\mathbf{R}$ surface to the $\mathbf{P}$ surface is necessarily nonadiabatic and there is no 'adiabatic' path [34]: the system must 'jump' from one solid curve to the other, unlike Fig. 1, in order to form directly the ground state products.

\section{IIE. Relaxation-limited rates}

Throughout this section and the previous one the rate expressions have been those which are appropriate to 'activation control', in which the probability of forming reactive configurations can be calculated from an equilibrium-type distribution function. That is, it is assumed that the probability of finding the system in the vicinity of the intersection region of Fig. 1 is a near-equilibrium one. However, under certain conditions the motion leading to the reactive configurations may partly involve very slow diffusive steps, either in the relative translational motion of the two reactants or in fluctuations of the environment itself, e.g., a slow diffusive-type dielectric relaxation. Then the overall reaction rate may be smaller than the above value. Indeed, there are a number of cases in the literature where the intrinsic rate of a bimolecular reaction is so high that the mutual diffusion together of the two reactants itself becomes the slow step. In this case, the preceding equations can be used in conjunction with an expression for a diffusion-controlled rate constant to obtain an expression for the overall reaction rate constant (Appendix IA). An example has also appeared (cited in Appendix ID) in which the slow step is a dielectric relaxation step, the 'activation controlled' step being very fast in that example. Several papers discussing such a possibility are cited in Appendix ID.

\section{Comparisons of predictions with experimental measurements on nonbiological systems}

\section{IIIA. Cross-reactions}

The most frequently used form of the theoretical equations has been what is now known in the literature as the 'cross-relation', Eqn. 10. If the work terms in Eqns. 2 and 3 are not neglected, the cross-relation, Eqn. 10, becomes Eqn. 22. The latter 
reduces to Eqn. 10, of course, when the work terms can be neglected:

$k_{12}=\left(k_{11} k_{22} K_{12} f_{12}\right)^{1 / 2} W_{12}$,

where now

$\ln f_{12}=\frac{1}{4} \frac{\left(\ln K_{12}+\frac{w_{12}-w_{21}}{R T}\right)^{2}}{\ln \left(\frac{k_{11} k_{22}}{Z^{2}}\right)+\frac{w_{11}+w_{22}}{R T}}$

$W_{12}=\exp \left[-\left(w_{12}+w_{21}-w_{11}-w_{22}\right) / 2 R T\right]$

and $Z$ was defined earlier $\left(=A \sigma_{11} \sigma_{22}\right) ; w_{12}$ is the work term for reaction $7, w_{21}$ is that for the reverse reaction and $w_{11}$ and $w_{22}$ are that for reactions 8 and 9 , respectively. As before, $K_{12}$ is the equilibrium constant expressed in terms of concentrations in the prevailing medium; it varies with salt concentration.

The work terms $w_{i j}$ can be of several types, not just electrostatic. One rather approximate (DebyeHückel) version for the electrostatic contribution to the work term is given by

$$
w_{i j}=\frac{\frac{z_{i} z_{j} \mathrm{e}^{2}}{2 D_{\mathrm{s}} \mathrm{r}}\left(\frac{\exp \left(B \sigma_{i} \sqrt{\mu}\right)}{1+B \sigma_{i} \sqrt{\mu}}+\frac{\exp \left(B \sigma_{j} \sqrt{\mu}\right)}{1+B \sigma_{j} \sqrt{\mu}}\right)}{\exp (B r \sqrt{\mu})}
$$

where $B=\left(8 \pi N \mathrm{e}^{2} / 1000 D_{\mathrm{s}} k T\right)^{1 / 2}, \sigma_{i}=a_{i}+a_{i}^{\prime}, \sigma_{j}$ $=a_{j}+a_{j}^{\prime}$, where $a^{\prime}$ is the radius of the dominant ion of opposite charge in the ionic atmosphere, and $z_{i}$ and $z_{j}$ are the charges of the reactants. For water at $25^{\circ} \mathrm{C} B$ equals $3.29 \mathrm{~nm}^{-1}$. Even if Eqn. 25 provides a first approximation to $w_{i j}$, a free-energy term, the corresponding entropy expression is probably quite poor, and should not be used to calculate the effect of ionic strength on the entropy of activation [78].

There have been numerous experimental tests of the cross-relation Eqn. 10, e.g., Refs. 79-83. Some representative examples are shown in Table I. The calculated rate constants were obtained neglecting the work terms. The entries illustrate the fact that the agreement between theory and experiment can be very good: the observed and

\section{TABLE I}

\section{COMPARISON OF OBSERVED AND CALCULATED RATE CONSTANTS FOR OUTER-SPHERE ELECTRON-TRANSFER REACTIONS}

The values of $k_{12, \text { calcd }}$ were calculated from Eqn. 10 neglecting the work terms; the calculated values are from the references cited and from Refs. 79-83. The references in the last column should be consulted for the experimental data.

\begin{tabular}{|c|c|c|c|c|}
\hline Reaction & $\log K_{12}$ & $\begin{array}{l}k_{12, o b s d} \\
\left(\mathrm{M}^{-1} \cdot \mathrm{s}^{-1}\right)\end{array}$ & $\begin{array}{l}k_{12, \text { calcd }} \\
\left(\mathrm{M}^{-1} \cdot \mathrm{s}^{-1}\right)\end{array}$ & Ref. \\
\hline $\mathrm{Ru}\left(\mathrm{NH}_{3}\right)_{6}^{2+}+\mathrm{Ru}\left(\mathrm{NH}_{3}\right)_{5} \mathrm{py}^{3+}$ & 4.40 & $1.4 \cdot 10^{6}$ & $4 \cdot 10^{6}$ & 79 \\
\hline $\mathrm{Ru}\left(\mathrm{NH}_{3}\right)_{5} \mathrm{py}^{2+}+\mathrm{Ru}\left(\mathrm{NH}_{3}\right)_{4}(\mathrm{bpy})^{3+}$ & 3.39 & $1.1 \cdot 10^{8}$ & $4 \cdot 10^{7}$ & 80 \\
\hline $\mathrm{Ru}\left(\mathrm{NH}_{3}\right)_{6}^{2+}+\mathrm{Co}(\text { phen })_{3}^{3+}$ & 5.42 & $1.5 \cdot 10^{4}$ & $1 \cdot 10^{5}$ & 81 \\
\hline $\mathrm{Ru}\left(\mathrm{NH}_{3}\right)_{5} \mathrm{Py}^{2+}+\mathrm{Co}(\text { phen })_{3}^{3+}$ & 1.01 & $2.0 \cdot 10^{3}$ & $1 \cdot 10^{4}$ & 81 \\
\hline $\mathrm{V}_{\mathrm{aq}}^{2+}+\mathrm{Co}(\mathrm{en})_{3}^{3+}$ & 0.25 & $5.8 \cdot 10^{-4}$ & $7 \cdot 10^{-4}$ & 81 \\
\hline $\mathrm{V}_{\mathrm{aq}}^{2+}+\mathrm{Ru}\left(\mathrm{NH}_{3}\right)_{6}^{3+}$ & 5.19 & $1.3 \cdot 10^{3}$ & $1 \cdot 10^{3}$ & 81 \\
\hline $\mathrm{Vaq}_{\mathrm{aq}}^{2+}+\mathrm{Fe}_{\mathrm{aq}}^{3+}$ & 16.90 & $1.8 \cdot 10^{4}$ & $2 \cdot 10^{6}$ & 81 \\
\hline $\mathrm{Fe}_{\mathrm{aq}}^{2+}+\mathrm{Os}(\mathrm{bpy})_{3}^{3+}$ & 1.53 & $1.4 \cdot 10^{3}$ & $5 \cdot 10^{5}$ & 81 \\
\hline $\mathrm{Fe}_{\mathrm{aq}}^{2+}+\mathrm{Fe}(\mathrm{bpy})_{3}^{3+}$ & 3.90 & $2.7 \cdot 10^{4}$ & $6 \cdot 10^{6}$ & 81 \\
\hline $\mathrm{Ru}\left(\mathrm{NH}_{3}\right)_{6}^{2+}+\mathrm{Fe}_{\mathrm{aq}}^{3+}$ & 11.23 & $3.4 \cdot 10^{5}$ & $2 \cdot 10^{6}$ & 84 \\
\hline $\mathrm{Ru}(\mathrm{en})_{3}^{2+}+\mathrm{Fe}_{\mathrm{aq}}^{3+}$ & 9.40 & $8.4 \cdot 10^{4}$ & $4 \cdot 10^{5}$ & 84 \\
\hline $\mathrm{Mo}(\mathrm{CN})_{8}^{4-}+\mathrm{IrCl}_{6}^{2-}$ & 2.18 & $1.9 \cdot 10^{6}$ & $8 \cdot 10^{5}$ & 85 \\
\hline $\mathrm{Mo}(\mathrm{CN})_{8}^{4-}+\mathrm{MnO}_{4}^{-}$ & -4.07 & $2.7 \cdot 10^{2}$ & $6 \cdot 10^{1}$ & 86 \\
\hline $\mathrm{Mo}(\mathrm{CN})_{8}^{4-}+\mathrm{HMnO}_{4}$ & 8.48 & $1.9 \cdot 10^{7}$ & $2 \cdot 10^{7}$ & 86 \\
\hline $\mathrm{Fe}(\mathrm{CN})_{6}^{4-}+\mathrm{IrCl}_{6}^{2-}$ & 4.08 & $3.8 \cdot 10^{5}$ & $1 \cdot 10^{6}$ & 85 \\
\hline $\mathrm{Fe}(\mathrm{CN})_{6}^{4-}+\mathrm{Mo}(\mathrm{CN})_{8}^{3-}$ & 2.00 & $3.0 \cdot 10^{4}$ & $4 \cdot 10^{4}$ & 85 \\
\hline $\mathrm{Fe}(\mathrm{CN})_{6}^{4-}+\mathrm{MnO}_{4}^{-}$ & 3.40 & $1.7 \cdot 10^{5}$ & $6 \cdot 10^{4}$ & 87 \\
\hline
\end{tabular}


calculated rate constants generally agree within an order of magnitude. There are some relatively minor trends: the data in the table are typical in that, with one exception, $k_{12 \text {,obsd }}<k_{12 \text {,calcd. How- }}$ ever, in almost all of these the $\mathrm{Fe}\left(\mathrm{H}_{2} \mathrm{O}\right)_{6}^{3+/ 2+}$ couple is one of the reactants (suggesting, as one possibility, that its mechanism for self-exchange may not be the same as that for the cross-reactions [83]). Good agreement is generally found when the couples involved are substitution inert. Unless corrected for work terms, deviations become larger when the reactants are oppositely charged. They can also be large when the driving force is large [88]. In other cases nonelectrostatic contributions to the work terms may be important. This appears to be the case, for example, in the cross-reactions of complexes with aromatic ligands (bipyridine or phenanthroline) with aquo ions: in these systems the calculated rates are 2-3 orders of magnitude faster than the observed rates. Noncancellation of the hydrophobic/hydrophilic interactions in the cross-relation (as well as departures from adiabaticity) have been considered as possible reasons for the breakdown in these unsymmetrical systems $[31,79,85,89]$. As mentioned above, in certain cases a change in mechanism (for example, from outersphere to inner-sphere) may be involved $[83,90]$.

\section{IIIB. Self-exchange reactions}

In the present section we apply the formalism to electron exchange rates of metal complexes in solution. Because the reactants are chemically similar to the products (merely an interchange of an electron) there are no net thermodynamic changes associated with the electron transfer; consequently, such reactions have an extra simplicity to them - no effect of $\Delta G^{0}$ need be included. In addition, in exchange reactions the molecular properties of only one redox couple rather than of two different redox couples need to be known.

The rates of a variety of electron exchange reactions are summarized in Table II. These reactions have been selected because they illustrate the range of application of the theory and because, as will be seen later, several of the couples are used as probes of metalloprotein reactivity. As expected, the electron exchange rates depend upon the nature of the metal center as well as upon the nature of the surrounding ligands. For a given metal center the rates tend to increase with the size of the ligands, reflecting the decrease in the magnitude of the solvent reorganization barrier with increasing size of the reactants, while, for a given set of ligands, the rate constants decrease with

TABLE II

COMPARISON OF OBSERVED ELECTRON-EXCHANGE RATE CONSTANTS WITH THE VALUES CALCULATED FROM THE OUTER-SPHERE, ADIABATIC MODEL

The value of the nuclear tunneling factor $\Gamma$ was calculated from

$\Gamma=\exp \left\{-\frac{\lambda_{\mathrm{i}}}{h \nu_{\mathrm{i}}}\left(\tanh \frac{\mathrm{h} \nu_{\mathrm{i}}}{4 k T}-\frac{h \nu_{\mathrm{i}}}{4 k T}\right)\right\}$

where $\nu_{i}$ is the symmetric breathing frequency. See Ref. 21 for details of the calculations of $k_{\text {calcd }}$ the values of $\lambda_{i} / 4$ and $\lambda_{0} / 4$ for the six reactions in Table II are $35.2,28.9,15.9,28.9 ; 3.3,28.0 ; 0,13.8 ; 73.7,28.5$ and $57.3,13.8 \mathrm{~kJ} \cdot \mathrm{mol}^{-1}$, respectively. The references in the last column should be consulted for the experimental data.

\begin{tabular}{|c|c|c|c|c|c|c|c|}
\hline Couple & $\begin{array}{l}\mu \\
(\mathrm{M})\end{array}$ & $\begin{array}{l}k_{\text {obsd }} \\
\left(\mathrm{M}^{-1} \cdot \mathrm{s}^{-1}\right)\end{array}$ & $\begin{array}{l}\Delta d_{0} \\
(\mathrm{~nm})\end{array}$ & $\begin{array}{l}r \\
(\mathrm{~nm})\end{array}$ & $\Gamma$ & $\begin{array}{l}k_{\text {calcd }} \\
\left(\mathrm{M}^{-1} \cdot \mathrm{s}^{-1}\right)\end{array}$ & Refs. \\
\hline $\mathrm{Fe}\left(\mathrm{H}_{2} \mathrm{O}\right)_{6}^{2+}+\mathrm{Fe}\left(\mathrm{H}_{2} \mathrm{O}\right)_{6}^{3+}$ & 0.55 & 4.2 & 0.014 & 0.65 & 3.2 & 3 & 78,91 \\
\hline $\mathrm{Ru}\left(\mathrm{H}_{2} \mathrm{O}\right)_{6}^{2+}+\mathrm{Ru}\left(\mathrm{H}_{2} \mathrm{O}\right)_{6}^{3+}$ & 1.0 & $\left(10^{2}\right)$ & 0.009 & 0.65 & 1.5 & $4 \cdot 10^{3}$ & 88,92 \\
\hline $\mathrm{Ru}\left(\mathrm{NH}_{3}\right)_{6}^{2+}+\mathrm{Ru}\left(\mathrm{NH}_{3}\right)_{6}^{3+}$ & 0.10 & $3 \cdot 10^{3}$ & 0.004 & 0.67 & 1.0 & $1 \cdot 10^{5}$ & 78,84 \\
\hline$R u(b p y)_{3}^{2+}+R u(b p y)_{3}^{3+}$ & 0.10 & $4 \cdot 10^{8}$ & 0.000 & 1.36 & 1.0 & $1 \cdot 10^{9}$ & 78,93 \\
\hline $\mathrm{Co}\left(\mathrm{NH}_{3}\right)_{6}^{2+}+\mathrm{Co}\left(\mathrm{NH}_{3}\right)_{6}^{3+}$ & 1.0 & $\geqslant 10^{-7}$ & 0.022 & 0.66 & 7.0 & $4 \cdot 10^{-6}$ & 78,94 \\
\hline $\mathrm{Co}(\mathrm{bpy})_{3}^{2+}+\mathrm{Co}(\mathrm{bpy})_{3}^{3+\mathrm{a}}$ & 0.1 & 18 & 0.019 & 1.36 & 5.0 & 20 & 78,95 \\
\hline
\end{tabular}

a The reduced force constant for the $\mathrm{Co}\left(\mathrm{NH}_{3}\right)_{6}^{3+/ 2+}$ couple was used in calculating the inner-sphere reorganization energy for the Co(bpy) $3^{3+/ 2+}$ couple. 
increasing difference in the metal-ligand distances in the two oxidation states.

Included in Table II are the rate constants calculated from the outer-sphere adiabatic model, but corrected (via $\Gamma$ ) for nuclear tunnelling effects [21]. Here, changes in the bond lengths and angles within the ligands themselves were not considered in calculating $\lambda_{\mathrm{i}}$. The $\lambda_{\mathrm{o}}$ values for the reactions were calculated assuming that $r$ is equal to the sum of the radii of the two reactants: support for this assumption is provided by the fact that the logarithm of the (precursor corrected) rate constants for the $\mathrm{Ru}\left(\mathrm{NH}_{3}\right)_{6}^{3+/ 2+}, \mathrm{Ru}\left(\mathrm{NH}_{3}\right)_{5}(\mathrm{py})^{3+/ 2+}$, $\mathrm{Ru}\left(\mathrm{NH}_{3}\right)_{4}(\mathrm{bpy})^{3+/ 2+}, \mathrm{Ru}\left(\mathrm{NH}_{3}\right)_{2}(\mathrm{bpy})_{2}^{3+/ 2+}$, and $\mathrm{Ru}(\mathrm{bpy})_{3}^{3+/ 2+}$ exchange reactions, in which the sizes of the reactants are systematically increased, show a linear dependence upon $1 / 2 a$ (Fig. 6) [96]. The slope is somewhat larger (30\%) than the theoretical value of $-7.6 \mathrm{~nm}$ (the agreement improves when inner-shell changes are included). The effect of change of metal-ligand bond lengths $\Delta d_{0}$ on $\Delta G^{*}$ is displayed in Fig. 7, where the rate constants for some thirteen self-exchange reactions are corrected via Eqn. 6 for the differences in $\lambda_{0}$ and plotted as $\ln \left(k_{\mathrm{ex}} / K_{\mathrm{A}}\right)+\left(\lambda_{\mathrm{o}} / 4 R T\right)$ vs. $\left(\Delta d_{0}\right)^{2}$ [78], where $K_{\mathrm{A}}$ is an encounter equilibrium con-

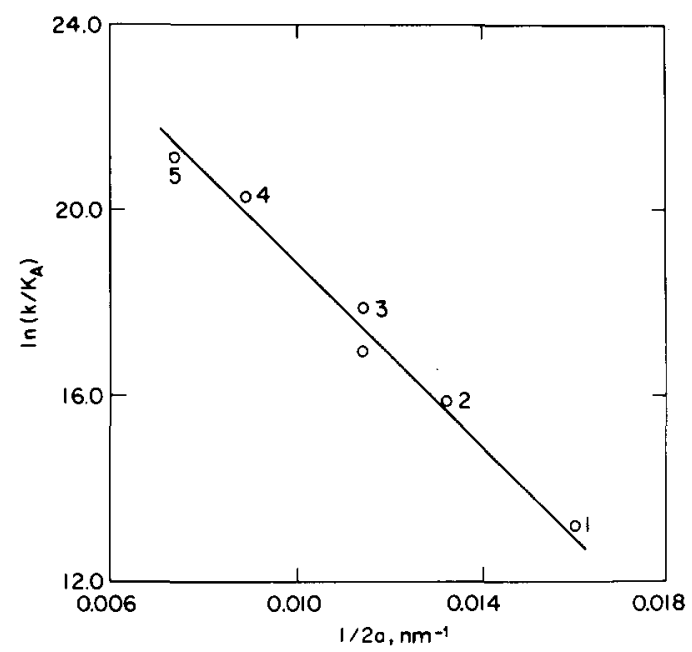

Fig. 6. Plot of the logarithm of the precursor-corrected exchange rate constants as a function of $1 / 2 a$. (1), $\mathbf{R u}\left(\mathrm{NH}_{3}\right)_{6}^{3+/ 2+} ; \quad$ (2), $\quad \mathbf{R u}\left(\mathrm{NH}_{3}\right)_{5}(\mathrm{py})^{3+/ 2+} ; \quad$ (3), $\mathrm{Ru}\left(\mathrm{NH}_{3}\right)_{4}(\mathrm{bpy})^{3+/ 2+} ;$ (4), $\mathrm{Ru}\left(\mathrm{NH}_{3}\right)_{2}(\mathrm{bpy})_{2}^{3+/ 2+}$; (5) Ru(bpy $)_{3}^{3+/ 2+}$. From Ref. 96 . stant (Appendix IA). The correlation is the one expected from Eqn. 5. The comparatively minor deviations are, in part, due to differences in the normal mode force constants and in $\Gamma$.

The dependence of the exchange rates on the solvent dielectric properties predicted by the model is found for some, but not all of the systems studied. Thus the predicted dependence on $\left(1 / D_{\mathrm{op}}\right.$ $-1 / D_{\mathrm{s}}$ ) is seen for the bis(biphenyl)chromium ${ }^{+/ 0}$ [97]. $\mathrm{Ru}(\mathrm{hfac})_{3}^{0 /-}, \mathrm{Ru}\left(\left(\mathrm{CH}_{3}\right)_{2}\right.$ bpy $)(\mathrm{hfac})_{2}^{+/-}$(hfac $=$ hexafluoroacetylacetonate ion) [98], and aromatic molecule-aromatic anion exchanges [99], but not for the $\mathrm{Fe}(\mathrm{cp})_{2}^{+/ 0}$ exchange [100] ( $\mathrm{cp}=$ cyclopentadienyl radical). An example of such a plot is given in Fig. 8. Specific ion-pairing and solvation effects or strong interaction of the reactants could be responsible for the absence of the predicted solvent dependence in the $\mathrm{Fe}(\mathrm{cp})_{2}^{+/ 0}$ system (where the ligand carries a net negative charge).

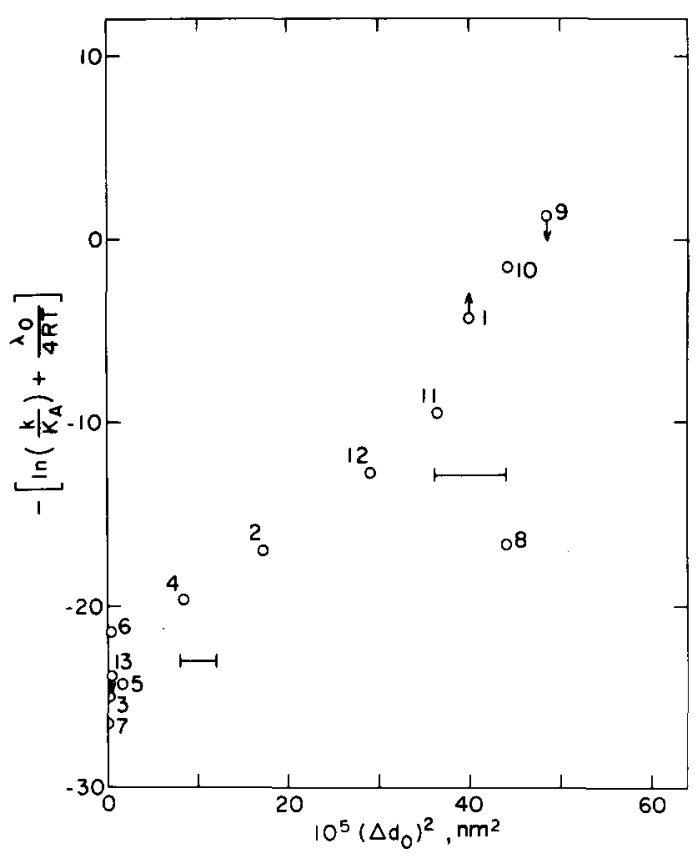

Fig. 7. Plot of the sum of $\lambda_{\mathrm{o}} / 4 R T$ and the logarithm of the precursor-corrected exchange rate constant as a function of $\left(\Delta d_{0}\right)^{2}$. (1), $\quad \mathrm{Cr}\left(\mathrm{H}_{2} \mathrm{O}\right)_{6}^{3+/ 2+} ; \quad(2), \quad \mathrm{Fe}\left(\mathrm{H}_{2} \mathrm{O}\right)_{6}^{3+/ 2+} ; \quad$ (3), $\mathrm{Fe}(\text { phen })_{3}^{3+/ 2+}$; (4), Ru( $\left.\mathrm{H}_{2} \mathrm{O}\right)_{6}^{3+/ 2+}$; (5), $\mathrm{Ru}\left(\mathrm{NH}_{3}\right)_{6}^{3+/ 2+}$; (6), $\mathrm{Ru}(\mathrm{en})_{3}^{3+/ 2+}$, (7), Ru(bpy) ${ }_{3}^{3+/ 2+} ;$ (8), $\mathrm{Co}\left(\mathrm{H}_{2} \mathrm{O}\right)_{6}^{3+/ 2+} ;(9)$, $\mathrm{Co}\left(\mathrm{NH}_{3}\right)_{6}^{3+/ 2+} ;(10), \mathrm{Co}(\mathrm{en})_{3}^{3+/ 2+} ;(11), \mathrm{Co}(\mathrm{bpy})_{3}^{3+/ 2+} ;$ (12), $\mathrm{Co}(\mathrm{sep})^{3+/ 2+}$; (13), Co(bpy) $)_{3}^{2+/+}$. From Ref. 78 . 


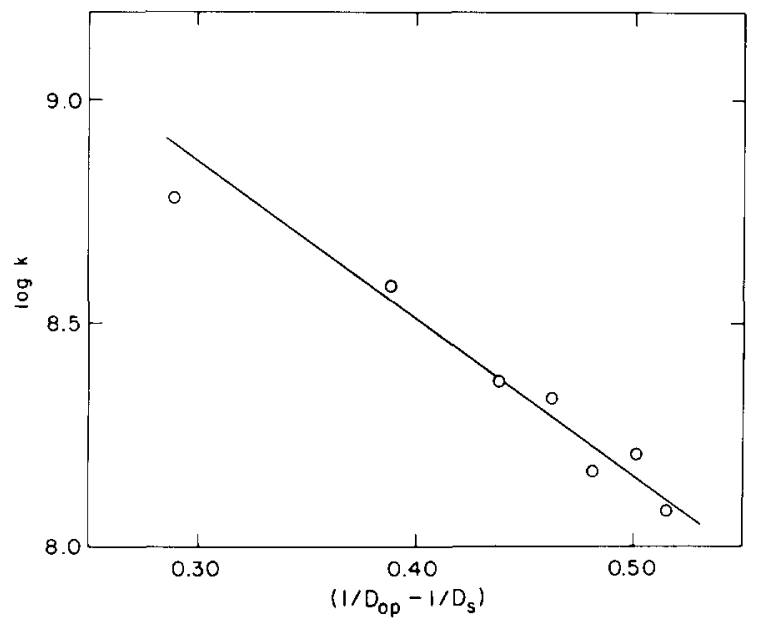

Fig. 8. Plot of the logarithm of the rate constant for the bis(biphenyl)chromium ${ }^{+/ 0}$ exchange reaction as a function of $\left(1 / D_{\mathrm{op}}-1 / D_{\mathrm{s}}\right)$. From Ref. 97.

In view of the generally excellent agreement of the observed and calculated exchange rate constants it is tempting to conclude that the model is essentially correct and, in particular, that the assumption that $\kappa \cong 1$ is justified for the systems considered. This assumption has been explored theoretically for a few systems. For example, in the case of ruthenium systems the spatial extension of the ruthenium $4 \mathrm{~d}$ orbitals and the electron delocalization on to the ligands through $\pi$-backbonding effects suggest that $\kappa$ for these exchanges is unlikely to be much less than unity [101]. Consistent with this conclusion, a value of $\kappa \approx 0.2$ has been calculated for the $\mathrm{Ru}\left(\mathrm{NH}_{3}\right)_{6}^{3+/ 2+}$ exchange reaction [77]. On the other hand, on the basis of direct $4 \mathrm{~d}-4 \mathrm{~d}$ overlap of the metal centers, the $\mathrm{Ru}(\mathrm{bpy})_{3}^{3+/ 2+}$ exchange would be highly nonadiabatic at the separation distance defined by first contact of the bipyridine ligands of the two reactants $\left(\kappa \approx 10^{-4}\right.$ based on a $\beta$ value in $\exp \left[-\beta\left(r-r_{0}\right)\right]$ of $\left.0.12 \mathrm{~nm}^{-1}\right)$. However, a $\kappa$ value of about unity has been suggested for the $\mathrm{Ru}(\mathrm{bpy})_{3}^{3+/ 2+}$ exchange by estimating the $\pi^{*}-\pi^{*}$ interaction of the two reactants resulting from the delocalization of the metal $\mathrm{d} \pi$ electron density onto the $\pi^{*}$ orbitals of the bipyridine ligands [101].

Possible nonadiabatic effects have also been explored in calculations for some of the other systems. Recent calculations of $\kappa(r)$ for the $\mathrm{Fe}\left(\mathrm{H}_{2} \mathrm{O}\right)_{6}^{3+/ 2+}$ exchange indicate that the optimum $r$ occurs at $0.525 \mathrm{~nm}$, appreciably smaller than the separation distance $0.65 \mathrm{~nm}$ defined by the effective radii of the reactants [76]. The calculations yield $\kappa(r) \approx 0.2$ at $r=0.525 \mathrm{~nm}$ compared with $\kappa(r) \approx 10^{-2}$ at $r=0.65 \mathrm{~nm}$. The dependence of $\kappa(r)$ on orientation at small $r$ 's $(0.525 \mathrm{~nm})$ introduces a steric factor there, as noted earlier.

In contrast to the ruthenium and iron exchanges, the cobalt exchanges are accompanied by a spin change and on this basis should proceed very slowly: $\kappa$ for the cobalt exchanges has been estimated to be $10^{-3 \pm 1}$ at $r=0.68 \mathrm{~nm}$ [21]. Despite this, the good agreement in Table II of the observed rate with the rate calculated assuming $\kappa \approx 1$ leaves very little room for any special nonadiabaticity in the cobalt exchanges. The same conclusion has also been reached in a more extensive comparison of rates, where it was found that the data for a variety of exchanges (including cobalt systems) are consistent with $\kappa \geq 10^{-2}$ [78]. Since many of the cobalt systems studied involve changes in spin quantum number, these results suggest that spin multiplicity restrictions per se are generally not of major importance in determining electron-transfer rates between transition metal complexes. For systems where the spin-orbit coupling is small, for example in electron-transfer involving organic systems, the spin restrictions can be more important and would reduce the value of $\kappa$.

Good agreement between the observed and calculated exchange rates is not always obtained: for example, the $\mathrm{Co}\left(\mathrm{H}_{2} \mathrm{O}\right)_{6}^{3+/ 2+}$ exchange reaction proceeds about $10^{7}$-times more rapidly than predicted [21]. Clearly, there is a more favorable pathway for this reaction than the simple outersphere exchange considered. Various possibilities have been proposed including a pre-equilibrium spin change [101] and an inner-sphere waterbridged mechanism [90].

IIIC. Relation between rates and equilibrium constants

We next consider reactions in which the electron transfer is accompanied by a net chemical change. For such systems the formalism predicts a 
linear relationship between $\log k$ and $\log K$ (denoted earlier by $\left.K_{12}\right)$ provided that $\left|\Delta G^{0 \prime}\right| \ll \lambda$. More generally, plots of $\log k$ vs. $\log K$ are predicted from Eqns. 1-3 to be curved with a local slope $\alpha$ (at any particular value of $\Delta G^{0 \prime}$ ) given by

$\alpha=\frac{1}{2}\left[1+\Delta G^{0 \prime} / \lambda\right]$

In a number of systems a linear relationship between $\log k$ and $\log K$ with a slope of $0.4-0.6$ is indeed observed (Table III). In systems encompassing large driving forces plots of $\log k$ vs. $\log$ $K$ are found to be curved as expected from Eqn. 26. Often, however, they are essentially linear over the restricted driving force ranges usually used. An example is the family of $\mathrm{RuL}_{3}^{+}-\mathrm{Eu}_{\mathrm{aq}}^{3+}$ reactions (different polypyridine ligands $\mathrm{L}$ ) for which $-\Delta G^{0}$ ranges from 1.2 to $1.5 \mathrm{eV}$, and which is predicted to have a slope of 0.28 ; the value observed $(0.12)$ is, however, even smaller. The decreased slope has been ascribed to effects of nonadiabaticity $[21,101]$. Good correlation between $\log k$ and $\log K$ has been found for a broad variety of metalloprotein electron-transfer reactions [111].

\section{TABLE III}

\section{SLOPE OF PLOT OF LOG $k$ VS. LOG $k$ FOR A SERIES OF RELATED ELECTRON-TRANSFER REACTIONS}

Data for electrochemical systems are presented in Appendix ID. The asterisks in the first column indicate electronically excited states of the complexes, and $\mathrm{HAs}^{-}$denotes the ascorbate anion, PTZ $N$-alkylphenothiazine derivatives, $\mathrm{SnR}_{4}$ tetraalkyltin derivatives, $\mathrm{P}^{2+}$ bipyridinium derivatives, and $\mathrm{ArNR}_{2}$ aromatic amines.

\begin{tabular}{|c|c|c|}
\hline Reaction & Slope & Refs. \\
\hline $\mathrm{FeL}_{3}^{3+}+\mathrm{Fe}_{\mathrm{aq}}^{2+}$ & 0.51 & $\overline{102}$ \\
\hline $\mathrm{FeL}_{3}^{2+}+\mathrm{Ce}(\mathrm{IV})$ & 0.44 & 103 \\
\hline $\mathrm{RuL}_{3}^{3+}+\mathrm{Fe}_{2 \mathrm{~g}}^{2+}$ & 0.51 & 89 \\
\hline $\mathrm{OsL}_{3}^{3+}+\mathrm{Ti}^{3+}$ & 0.37 & 104 \\
\hline${ }^{*} \mathrm{RuLL}_{3}^{2+}+\mathrm{Eu}_{\mathrm{aq}}^{3+}$ & 0.49 & 89 \\
\hline${ }^{*} \mathrm{CrL}_{3}^{3+}+\mathrm{Fe}_{\mathrm{aq}}^{2+4}$ & 0.43 & 105 \\
\hline $\mathrm{FeL}_{3}^{3+}+\mathrm{HAs}^{-}$ & 0.50 & 106,107 \\
\hline $\mathrm{Fe}_{\mathrm{aq}}^{3+}+\mathrm{PTZ}$ & 0.50 & 108 \\
\hline $\mathrm{Fe}(\mathrm{phen})_{3}^{3+}+\mathrm{SnR}_{4}$ & 0.50 & 109 \\
\hline $\mathrm{P}^{2+}+{ }^{*} \mathrm{Ru}(\mathrm{bpy})_{3}^{2+}$ & $0.50^{\mathrm{a}}$ & 110 \\
\hline${ }^{*} \mathrm{Ru}(\mathrm{bpy})_{3}^{2+}+\operatorname{ArNR}_{2}$ & $0.50^{\mathrm{a}}$ & 110 \\
\hline
\end{tabular}

\footnotetext{
In acetonitrile.
}

\section{IIID. Mixed-valence systems}

Mixed-valence complexes in which two metal centers are connected by a bridging group provide systems that are very useful for the comparison of theoretical and experimental parameters. Not only are the theoretical problems associated with the formation of the precursor complex eliminated, but the distance separating the metal centers is also known. In addition, many mixed-valence systems feature an intervalence absorption band in the near-infrared region of the spectrum and $H_{\mathrm{AB}}$, $\lambda_{i}$ and $\lambda_{o}$ can be obtained from the intensity, energy and solvent dependence of this band $[112,113]$.

The energy of the intervalence transition $E_{\text {op }}$ is equal to $\lambda+\Delta G^{0 \prime}[114]^{~ ", ~ w h e n c e ~ t h e ~} \Delta G^{*}$ in Eqn.

\footnotetext{
In Refs. 112 and $113 E_{\mathrm{op}}$ is assumed to be $\lambda+\Delta E^{0}$, where $\Delta E^{0}$ is the internal energy change. However, in Ref. 114 the transition energy is shown to be a free-energy change $\lambda+$ $\Delta G^{0}$. The equality of $E_{\text {op }}$ and $\lambda+G^{0}$ is evident from Eqn. 14 of Ref. 114, where $F_{\mathrm{e}-\mathrm{g}}^{\mathrm{op}}-F_{\mathrm{e}-\mathrm{g}}$ is the polar contribution to $\lambda$ and $F_{\mathrm{e}}-F_{\mathrm{g}}$ is the polar contribution to $\Delta F^{0}$ ( $\Delta G^{0}$ in the present notation). In more physical terms, the reason why $E_{\text {op }}$ equals $\lambda+\Delta G^{0 \prime}$ rather than $\lambda+\Delta E^{0 \prime}$ can be seen from the following argument. According to the Franck-Condon principle, the momenta and positions of the nuclei do not change during the vertical transition. Consequently, the instantaneous distribution of momenta and coordinates is the same immediately before and just after the optical transition and there is no change in the entropy during the vertical transition (apart from entropy changes associated with changes in electronic multiplicity). Let the vertical transition energy $E_{\text {op }}$ be denoted by $\lambda$ when $\Delta G^{0 \prime}=0$. At $\Delta G^{0 \prime}=0$, we have $\Delta H^{0 \prime}=T \Delta S^{0}$, and so $E_{\text {op }}$ can be written equivalently as $\lambda+\Delta H^{0 \prime}-T \Delta S^{0 \prime}$. If, now, the products' potential-energy surface is vertically displaced downward, $\Delta S^{0}$ is unchanged, but $\Delta H^{0 \prime}$ is made more negative. The $E_{\text {op }}$ is now $\lambda$ plus this (negative) change in $\Delta H^{0 \prime}$. In other words, when $\Delta G^{0 \prime} \neq 0$, we can still write $E_{\text {op }}$ as $\lambda+\Delta H^{0 \prime}-T \Delta S^{0}$, i.e., as $\lambda+\Delta G^{0}$, as in the text. The $\lambda$ defined above can be related to the free-energy barrier in the thermal electron transfer as follows: according to the key approximation made in deriving Eqns. 1 to 3, the free energy of each of the surfaces is a quadratic function (actually of a quantity $m$ in Refs. 1-3), and it follows from this property that when $\Delta G^{0 \prime}=0$ the free-energy change for reaching the intersection is one fourth the free energy change for the vertical transition. Since, as we have already noted, there is no change in entropy during the vertical transition, the vertical change in free-energy equals that in energy, namely $E_{\text {op. }}$. Thus, the free-energy barrier for the thermal transfer is $E_{\mathrm{op}} / 4$, i.e., $\lambda / 4$.
} 
2 becomes Eqn. 27 (apart from the $w^{\mathrm{r}}$ in Eqn. 2, which is now absent)

$\Delta G^{*}=E_{\mathrm{op}}^{2} / 4\left(E_{\mathrm{op}}-\Delta G^{0 \prime}\right)$.

The $\Delta H^{*}$ and $\Delta S^{*}$ for this first-order reaction are defined by the standard thermodynamic relations

$\Delta H^{*}=\left(\frac{\partial\left(\Delta G^{*} / T\right)}{\partial(1 / T)}\right)_{p}$
$\Delta S^{*}=-\left(\frac{\partial \Delta G^{*}}{\partial T}\right)_{p}=\frac{\Delta H^{*}-\Delta G^{*}}{T}$

with $\Delta G^{*}$ given by Eqn. 2 or, for the present system, by Eqn. 27a. Eqn. 27a and b yield [115]:

$\Delta H^{*}=\frac{E_{\mathrm{op}}\left[E_{\mathrm{op}}+2\left(\Delta H^{0 \prime}-\Delta G^{0 \prime}\right)\right]}{4\left(E_{\mathrm{op}}-\Delta G^{0 \prime}\right)}$,

and $\Delta S^{*}$ then equals $\frac{1}{2} \Delta S^{0 \prime} E_{\mathrm{op}} /\left(E_{\mathrm{op}}-\Delta G^{0 \prime}\right)$. ( $\Delta H^{*}$ and $\Delta G^{*}$ have been confused in certain applications involving $E_{\text {op }}$ in the literature, and Eqn. 27d is new [115].

If the intervalence band has a Gaussian shape and the degree of ground-state delocalization is small, then $H_{\mathrm{AB}}$ is related to the properties of the band by $[112,113]$ :

$\left|H_{\mathrm{AB}}\right|=\frac{2.06 \cdot 10^{-3}}{r}\left(\epsilon_{\max } \bar{\nu}_{\max } \Delta \bar{\nu}_{1 / 2}\right)^{1 / 2} \mathrm{~cm}^{-1}(28)$

where $r$ is in $\mathrm{nm}$ and $\nu$ in wavenumbers. At room temperature the energy and half-width of a Gaussian-shaped band (for the case $\Delta G^{0 \prime}=0$ ) are related by Refs. 112 and 113 (cf.Ref. 114):

$\Delta \bar{\nu}_{1 / 2}=\left(2.31 \cdot 10^{3} \bar{\nu}_{\max }\right)^{1 / 2} \mathrm{~cm}^{-1}$

(When $\Delta G^{0 \prime} \neq 0$ the $\bar{\nu}_{\text {max }}$ in Eqn. 29 is replaced by $\bar{\nu}_{\max }-\Delta G^{0^{\prime}}$, where $\Delta G^{0^{\prime}}$ is now in units of $\mathrm{cm}^{-1}$.) Consequently, the half-width can be eliminated from Eqn. 28 which then contains only $r, \epsilon_{\max }$, and $\bar{\nu}_{\max }$ as parameters. Another way of obtaining $H_{\mathrm{AB}}$ is from EPR, namely from the singlet-triplet splitting of a radical pair $[64,116,117]$ : such a method yields a value for the electron-transfer matrix element $H_{\mathrm{AB}}$ when the splitting is predomi- nantly due to the presence of a singlet chargetransfer state [116,117]. The latter affects the energy of the singlet, but not of the triplet, radical pair.

The application of Eqns. 27a, 28 and 29 will be illustrated by considering the electron transfer within mixed-valence diruthenium systems. There is reasonable agreement between $\bar{\nu}_{\max }\left(9.7 \cdot 10^{3}\right.$ $\mathrm{cm}^{-1}$ ) for the 4,4'-bipyridine-bridged system and the value calculated $\left(12.8 \cdot 10^{3} \mathrm{~cm}^{-1}\right)$ using a model in which each half of the binuclear ion is approximated by a sphere of radius $0.37 \mathrm{~nm}[96,118]$. Similarly, the solvent dependence of the intervalence band (slope of $\bar{\nu}_{\max }$ vs. $\left(1 / D_{\mathrm{op}}-1 / D_{\mathrm{s}}\right)$ ) is only $30 \%$ smaller than the value calculated from

$E_{\mathrm{op}}=\lambda_{\mathrm{i}}+\lambda_{\mathrm{o}}$

where $\lambda_{\mathrm{i}}$ and $\lambda_{\mathrm{o}}$ are given by Eqns. 5 and 6, with $a=0.37 \mathrm{~nm}$ and $r=1.08 \mathrm{~nm}$. The value of $H_{\mathrm{AB}}$ calculated from the intensity of the intervalence band $[119,120]$ is $400 \mathrm{~cm}^{-1}$, corresponding to $\kappa \approx 1$. Introducing a $-\mathrm{CH}_{2}$-group between the two pyridine rings reduces the coupling of the metal centers, so that the electron transfer becomes somewhat nonadiabatic $(\kappa=0.2)$. Nevertheless, the coupling in the latter system is still very large compared with the through-space coupling of two ruthenium centers a distance 1.0-1.2 nm apart for which $\kappa \approx 10^{-4}$ has been estimated as discussed above.

In the Creutz-Taube ion [121] the two ruthenium centers are connected by a pyrazine group. This group provides strong electronic coupling of the metal centers instead of weak coupling. The properties of the pyrazine-bridged system are those expected for a delocalized rather than a localized ground state, (and so Eqn. 6 becomes inapplicable). The near-infrared band of the Creutz-Taube ion does not show the solvent dependence expected for weak overlap intervalence systems and, in contrast to the band of the bipyridine-bridged system, the near-infrared band of the pyrazinebridged complex is much narrower than predicted by Eqn. 29 [113]. This is an interesting system and the proper description of the Creutz-Taube ion is still a subject of debate [122]. A three-site electronic-nuclear model has been discussed recently 
for such strongly delocalized bridged systems [123-125].

Although a bridging group is useful, it is not required for the observation of an intervalence absorption band: such bands are also observed in outer-sphere ion pairs and can be analyzed in terms of the above two-site formalism [126].

Experimental [127,128] and theoretical [71] studies of the dependence of absorption intensity of charge-transfer spectra on distance along fixed bridges have been described recently. A dependence of $H_{\mathrm{AB}}$ on the coordinates (e.g., in Figs. 1-4) has also been considered [129]. Such a dependence can cause the matrix elements for the thermally and optically induced electron transfer to differ [129]. However, when a realistic model is used instead of a delta function or a square-well potential, the difference between the matrix elements becomes much smaller [71].

A phenomenon related to charge-transfer spectra is the photoionization of ions in solution $[130,131]$. The photoionization spectrum of each ion is related to the value of $\lambda$ plus the $\Delta G^{0}$ for this process [131].

\section{IIIE. Electrode reactions}

There has been an extensive comparison of the self-exchange rates with the rates of the corresponding reactions at an electrode, determined at zero activation overpotential in order to test the relation predicted in Eqn. 11 (e.g., Refs. 17, 132,133 contain recent summaries). There is a good correlation at lower rates, but at the highest rates the available data for the electrochemical rates appear to show a levelling-off.

There have been experimental studies, e.g., cited in Appendix ID, of the theoretical analog [3,29-31] of Eqn. 26 for electrode reactions in which the activation overpotential $n e\left(E-E_{0}^{\prime}\right)$ replaces $\Delta G^{0^{\prime}}$. ( $E$ is the half-cell potential, $E_{0}^{\prime}$ the 'standard' half-cell potential in the prevailing medium, and $n$ is the number of electrons, of charge $e$, transferred between reactant and electrode in the elementary step.) Recent studies of the curvature of plots of $1 n$ $k$ vs. $E-E_{0}^{\prime}$ have also been made $[134,135]$. They yield approximate agreement of the theoretical and calculated $E-E_{0}^{\prime}$ values for onset of significant deviation of $\alpha$ in Eqn. 26 from 0.5 .

\section{Extension to biological electron transfers}

\section{IVA. Similarities and differences with small mole- cule electron transfers}

We first note that biological electron transfers have certain features in common with the small molecule electron transfers, as well as features which are different. As in the simpler reactions, the reactants in biological systems can be expected to undergo vibrational and solvational (or environmental) changes during the reaction, and so the rates should depend on $\lambda$ and $\Delta G^{0}$. However, substantial differences also exist. (1) Whereas it was possible in the solution case to find self-exchange reactions for predicting the rate of a crossreaction, the former may sometimes not even exist in the biological case. (2) Whereas the structural information (bond length changes) needed for the prediction of absolute rate constants of self-exchange reactions is becoming increasingly available in the case of reactions in solution (particularly with the introduction of EXAFS) as are the relevant vibrational frequencies, such information is at present only fragmentarily available for most biological systems. Indeed, in some cases even the identity of all the coordinated ligands may not be known. (3) The environment (solvent) for the electron-transfer reactions in solution, which affects $\lambda$, is at present better known than that, for example, in protein systems, which are structurally less homogeneous. (4) Whereas $\Delta G^{0}$ is usually quite well-known for reactions in solution, knowledge of it for the case where the pair of reactants are either in, or attached to, a membrane is frequently less certain". (5) Quantities such as $\Delta G^{0}$ now become dependent on any electric field which has developed across the membrane. (6) Protein-conformational changes may precede or follow the electron transfer in biological systems. For example, such conformational changes may modify relative binding free energy of the oxidized and the reduced

\footnotetext{
"Further, in applications of the theoretical expressions to an electron transfer step (both in a biological and a nonbiological reaction) one also needs to be particularly careful that the $\Delta G^{0}$ used refers only to that step and hence that any experimental value is corrected, when necessary, for any loss or gain of protons.
} 
forms of a substrate to the protein. (7) Whereas most electron-transfer reactions in solution are expected to occur at or near contact between the reactants, with a reaction probability which depends on $\lambda$ and $\Delta G^{0}$, reactants in a biological membrane are frequently fixed in position, and so are prevented from coming in contact. (In this respect the biological reactants are similar to those in long range non-biological intramolecular electron-transfer reactions.) Their rate is expected to vary roughly as $\exp (-\beta r)$, i.e., to decrease exponentially with separation distance $r$ of the reactants and the rate now depends not only on the intrinsic $(\lambda)$ and thermodynamic $\left(\Delta G^{0}\right)$ factors, but also on $\beta$ and on the mutual orientation of the reactants. It also depends on $r$ through the outer $\left(\lambda_{0}\right)$ contribution to $\lambda$. Thereby, $\beta$ and $r$ become important parameters for any proper understanding of the rates.

Before proceeding to a discussion of some individual reactions, we first consider one general aspect of biological electron transfers: they often occur via a series of intermediates at fixed sites, for example in the charge transfer across a membrane for the photosynthetic reaction center (subsection VD). There are at least two factors which influence the choice of a mechanism involving several reaction intermediates versus one involving a single giant step. To illustrate these factors we consider a simple example of a system $a b c$ with one intervening site $b$. For transfer to occur in one hop (e.g., for mediation by b to occur via a superexchange mechanism without a well-defined intermediate $b^{-}$being formed), there is first a reorganization of the nuclear configuration of the end sites a and c, to make their energies equal, the system thereby reaching the intersection region in Fig. 1. The vertical energy difference of site $b$ and end sites a and $\mathrm{c}$ is denoted by $V . H_{\mathrm{abc}}$ is the effective electronic matrix element coupling a and $\mathrm{c}$ and in a superexchange mechanism (Appendix IC) equals $H_{\mathrm{ab}} H_{\mathrm{bc}} / V$, when $V \gg H_{\mathrm{ab}}$ and $H_{\mathrm{bc}}$.

\footnotetext{
This result, which is used here for illustrative purposes only, applies when there is only one orbital per bridge. In general, there is a sum of these $H_{\mathrm{ab}} H_{\mathrm{bc}} / V$ terms over the orbitals of the bridge, and $H_{\mathrm{ab}}, H_{\mathrm{bc}}$ and $V$ vary with the bridge orbital $\nu$. See, for example, Eqn. 15 of Ref. 70, where $H_{\mathrm{ab}}=\eta_{1} c_{1 \nu}$, $H_{\mathrm{bc}}=\eta_{2} c_{n \nu}$ and $V=b_{\nu}-a$, in the notation of Ref. 70 .
}

The site $\mathrm{b}$ poses little additional barrier only when $H_{\text {bc }} / V$ is not too small, and the transfer then occurs in a single hop. A single hop has the advantage for a downhill reaction of utilizing in full the overall $-\Delta G^{0^{\prime}}$, typically then requiring less reorganization free energy in the normal region, in accordance with Eqns. 1-3. On the other hand, when $H_{\mathrm{bc}} / V$ is very small, the transfer will occur in two successive hops provided that the formation of an actual intermediate $b^{-}$is not too 'uphill'. The matrix element for the first hop is now $H_{\mathrm{ab}}$, and is $H_{\mathrm{bc}}$ for the second hop. The $-\Delta G^{0^{\circ}}$ for at least one of these hops is now less favorable than the overall $-\Delta G^{0^{\prime}}$, and so may require a somewhat larger free energy of reorganization. Thus, the actual choice of mechanism depends on the relative contributions of the $\Delta G^{0^{\prime}}$ and $H_{\mathrm{bc}} / V$ factors discussed above.

\section{IVB. Reorganization parameters}

We next examine how the equations of Section II might be modified so as to treat reactions of metalloproteins, for instance those between cytochrome $c$ and various inorganic redox reagents. In applying Eqns. 1-4 the detailed description of the $\lambda_{o}$ and $\lambda_{i}$ contributions to the biological system (cytochrome $c$ in the present case) may differ from those for the ions in solution, and the magnitude of $\kappa$ becomes a particularly important question if there is an appreciable distance between the two electron-transfer sites. There may also be contributions to $w$ or $\lambda_{\mathrm{i}}$ arising from conformational changes.

The geometry of most metalloproteins, particularly as regards the dielectric situation, differs from that used to derive Eqn. 6 (although one might use Eqn. 6 with some mean $D_{\mathrm{s}}$ for the protein and/or membrane system). A more elaborate model to calculate $\lambda_{o}$ [137] can, of course, be introduced. In the present article we explore the possibility of using Eqns. 1-3. This we do in Section V, by first exploring the applicability of the cross-relation, Eqn. 10. Eqn. 2 has been used for cytochrome $c$ recently (apart from $w^{\mathrm{r}}$ ) [137]. The authors made an estimate of the reorganization parameter $\lambda$ (Eqn. 4) using a molecular model for the protein [137]. Electron transfer between most reactants in biological systems oc- 
curs between spatially fixed and oriented sites. In this case the reaction is formally a first-order one. The first-order rate constant $k$ can then be written as

$k=\kappa(r) \nu \exp \left(-\Delta G_{r}^{*} / R T\right)$

$\Delta G_{r}^{*}=\frac{\lambda}{4}\left[1+\frac{\Delta G^{0^{\prime}}}{\lambda}\right]^{2}$

where $\lambda$ again equals $\lambda_{i}+\lambda_{o}, \Delta G_{r}^{*}$ and $\Delta G^{0^{\prime}}$ are the free energy barrier and the free energy of reaction, respectively, when the reactants are a distance $r$ apart in the prevailing medium, and $\nu$ has dimensions of a frequency $\left(\mathrm{s}^{-1}\right)$. In the adiabatic regime $\nu$ is an effective frequency for nuclear motion along the reaction coordinate in Fig. 1 (discussed in Appendix I). In the nonadiabatic regime $(\kappa \ll 1), \kappa \nu$ is actually independent of the frequency of nuclear motion: comparison of Eqn. 31 with Eqn. 17 shows that when $\kappa \ll 1$, $\kappa \nu$ is given by:

$\kappa(r) \nu=2 \pi \frac{H_{\mathrm{AB}}^{2}}{\hbar(4 \pi \lambda R T)^{1 / 2}}$

As discussed later $H_{\mathrm{AB}}^{2}$ decreases exponentially with separation distance for many systems and so we shall write $\kappa(r) \nu$ as

$\kappa(r) \nu=1 \cdot 10^{13} \exp \left[-\beta\left(r-r_{0}\right)\right] \mathrm{s}^{-1}$

where $r_{0}$ is the value of $r$ at which $\kappa \nu$ equals some preassigned value, $1 \cdot 10^{13} \mathrm{~s}^{-1}$. Some experimental results in nonbiological systems place $\beta$ at about $12 \mathrm{~nm}^{-1}$ for aromatic molecules, and perhaps less if one of the states is electronically excited (cf. Section IVC). The values of $\beta$ are likely to vary from system to system somewhat, depending, for example, on the vertical ionization potential of the redox site and its determination for various model systems, for reactions of ground states, electronically excited states, anions, and cations, and its dependence on the intervening material present a central experimental problem in this field.

If the frequency for motion of the nuclei along the reaction coordinate is $1 \cdot 10^{13} \mathrm{~s}^{-1}$, a typical vibrational frequency, then in using Eqns. 31-33, one is tacitly assuming that the reaction is 'adiabatic' at the separation distance $r=r_{0}$, specifically that when $r=r_{0}$ the system moves from the $\mathrm{R}$ to the $P$ surface upon every passage through the nuclear configuration defining the intersection region in Fig. 1 (cf Appendix IA). Strictly speaking, the $\kappa$ in Eqn. 33 need not immediately fall exponentially with $r$ for $r \geq r_{0}$ : there could be some small interval for $r$, for which $\kappa$ is roughly constant and then the $\kappa(r)$ changes smoothly to an approximately exponential decrease with $r$. In bimolecular reactions the separation distance where the reaction is adiabatic is frequently assumed to correspond to close contact of the two reactants.

For membrane-bound systems the $\Delta G^{0^{\prime}}$ which appears in Eqn. 32 is for the conditions prevailing in the membrane and as such, can vary with the nearby charges present and with the electric fields they produce. If the electric field produces a given potential $\psi_{\mathrm{A}}$ at the charge center $\mathrm{A}$ and $\psi_{\mathrm{B}}$ at charge center $\mathrm{B}$, then $\Delta G^{0^{\prime}}$ can be written as [24]:

$\Delta G^{0^{\prime}}=\Delta G_{0}^{0}+\Delta e_{\mathrm{A}}\left(\psi_{\mathrm{B}}-\psi_{\mathrm{A}}\right)$,

where $\Delta e_{\mathrm{A}}$ is the (positive) charge transferred from site $A$ to site $B$. This equation presupposes that there is no significant change of the potential $\psi$ itself when the electron transfer occurs. $\Delta G_{0}^{0}$ is the value of $\Delta G^{0^{\prime}}$ in the absence of $\psi$.

Eqns. 31-33 have a number of applications. For example, one may vary $\Delta G^{0^{\prime}}$ by systematically varying one of the reactants. If $\lambda$ also varies, then an estimate of its variation is also needed (e.g., from self-exchange data). We discuss examples for bimolecular reactions in Section VA.

A second application is to the activation energy $E_{\mathrm{a}}$ of a first-order reaction. $E_{\mathrm{a}} / R$ is defined as the slope of the $\ln k$ vs. $1 / T$ plot, i.e., it equals $-\partial \ln$ $k / \partial(1 / T)$. From Eqns. 27b, 31 and 32, $E_{\mathrm{a}}$ for a first-order reaction is therefore given approximately by:

$E_{\mathrm{a}} \approx \Delta H_{r}^{*} \approx \frac{\lambda}{4}+\frac{\Delta H^{0^{\prime}}}{2}\left[1+\frac{\Delta G^{0^{\prime}}}{\lambda}\right]-\frac{\left(\Delta G^{0^{\prime}}\right)^{2}}{4 \lambda}$

when $\lambda$ and $\kappa \nu$ are assumed independent of temperature. In the normal region the magnitude of 
$\Delta G^{0^{\prime}} / \lambda$ in Eqn. 35 is less than unity. We see that when $\Delta H^{0^{\prime}}$ is very negative, the activation energy can even be negative. We have discussed elsewhere [60] such an application of Eqn. 35.

The first-order rate constant $k$ can be written as

$k=A^{\prime} \exp \left(-E_{\mathrm{a}} / R T\right)$,

where $E_{\mathrm{a}}$ is given by Eqn. 35 . Using Eqns. 31 and 35 the preexponential factor $A^{\prime}$ is given by

$A^{\prime}=\kappa(r) \nu \exp \left(\Delta S_{r}^{*} / R T\right)$,

where $\Delta S_{r}^{*}$ is obtained from the thermodynamic relation, Eqn. 27c (with $r$ subscripts).

It is sometimes useful in discussions to introduce a term, the 'entropy of activation' $\Delta S^{\dagger}$, which includes both $\Delta S_{\mathrm{r}}^{*}$ and an entropic contribution from $\kappa \nu$, namely ${ }^{\pi} R \ln \left(A^{\prime} / 10^{13}\right)$, where $A^{\prime}$ is in $\mathrm{s}^{-1}$. Thereby,

$\Delta S^{\dagger}=\Delta S_{\mathrm{r}}^{*}+\ln \left(\kappa \nu / 10^{13}\right)$,

where $\kappa \nu$ is in $s^{-1}$. Using Eqns. 27c, 32 and 35, $\Delta S_{\mathrm{r}}^{*}$ can be related to $\Delta S^{0^{\prime}}$, the standard entropy of reaction when the reactants are a distance $r$ apart in the prevailing medium, namely $\left(\Delta H^{0^{\prime}}-\right.$ $\left.\Delta G^{0^{\prime}}\right) / T$, as follows:

$\Delta S_{r}^{*}=\frac{1}{2} \Delta S^{0^{\prime}}\left(1+\Delta G^{0^{\prime}} / \lambda\right)$

Eqn. 36c and d are applied later.

We shall have occasion to refer to the 'barrierless regime', namely the region where $\Delta G^{0^{\prime}}$ is sufficiently negative that $-\Delta G^{0^{\prime}} \approx \lambda$. In this case, one sees from Eqns. 32, 35 and, with $r$ subscripts, $27 \mathrm{c}$ that $\Delta G_{r}^{*}, \Delta H_{r}^{*}$ and $\Delta S_{r}^{*}$ are each approx. zero. In particular, the activation energy $E_{\mathrm{a}}$ is approx. zero. (This point is also discussed in Appendix II.)

In the case of bimolecular reactions it is con-

\footnotetext{
'A closely related definition of an 'entropy of activation' is frequently used in the literature. For example, the $A^{\prime}$ in Eqn. $36 \mathrm{a}$ is of ten equated to $\left(k_{\mathrm{B}} T / h\right) \exp \left(\Delta S^{\dagger} / R\right)$, where $k_{\mathrm{B}}$ is Boltzmann's constant. However, since $k_{\mathrm{B}} T / h$ is approximately $1 \cdot 10^{13} \mathrm{~s}^{-1}$ at room temperature, this definition is equivalent to that used in the text for reactions studied under typical conditions.
}

venient to use a formalism for the rate constant based on the fixed site first-order rate constant $k$ in Eqn. 36a and a pre-equilibrium constant $K_{\mathrm{A}}$ for a precursor complex, described in Appendix I and used in Section V.

IVC. Electron tunneling barriers. Distance dependence of electron-transfer rate in nonbiological systems

We next present a brief description of determinations of $\beta$ in Eqn. 33 from nonbiological data. Several methods have been used:

(1) The first is based on the study of electrontransfer reactions in frozen media. Initially, there is a random distribution of the reactants. Since the closer pairs react first, the subsequent distribution is no longer random: consistent with Eqn. 33 the pairs at a distance $r$ have a rate constant $k(r)$ given by

$$
k(r)=k_{0} \exp \left[-\beta\left(r-r_{0}\right)\right]
$$

Using an expression for the fraction of pairs remaining at time $t$ and at each $r$ and then integrating over $r$, an expression for the fraction of unreacted material at time $t$ is obtained. Fitting the experimental data to the expression yields values of $k_{0}$ and of $\beta$. In many reactions between solvated electrons and various acceptors $\beta$ varied between 10 and $20 \mathrm{~nm}^{-1}$ (data not listed in Table IV, but summarized in Ref. 138). In electron-transfer reactions of biphenyl anions with some aromatic molecules [139], the anions being formed by pulse radiolysis, $\beta$ was calculated [138] from the data, and is given in the Table. Results for other systems are also given in Ref. 138, and most recently in Ref. 37 where a value of $\beta=12 \mathrm{~nm}^{-1}$ has been obtained (Table IV). Studies of hole transfer in frozen media were also reported [141], namely from pyrene $^{+}$and biphenyl ${ }^{+}$to tetramethylenephenylenediamine (cf. Table IV).

(2) Monolayers of fatty acids have been deposited on an aluminum layer, and then another metal layer deposited to form a capacitor, cf. [147] and references cited therein. Conductivities were measured for monolayers of fatty-acid salts containing 14-23 carbon atoms, the second metal being mercury [143], magnesium [144], gold [144], 
TABLE IV

SOME TENTATIVE VALUES OF $\beta$ IN THE LITERATURE

\begin{tabular}{|c|c|c|c|}
\hline System & $\beta\left(\mathrm{nm}^{-1}\right)$ & Comment & References \\
\hline Biphenyl-aromatics a & 12 & frozen media & 37 \\
\hline Biphenyl ${ }^{-}$-naphthalene & 12 & frozen media & 138,139 \\
\hline Biphenyl $^{-}$-phenylethylene & 10 & frozen media & 138,139 \\
\hline Biphenyl $^{-}$-acridine & 11 & frozen media & Miller, J.R., personal communication \\
\hline Pyrene $^{+}$-TMPD ${ }^{b}$ & 11.5 & frozen media & 141 \\
\hline Biphenyl $^{+}-$TMPD $^{b}$ & 11.5 & frozen media & 141 \\
\hline Biphenyl ${ }^{-}$-cinnamoyl grp. & $(<10)$ & steroid bridged & 142 \\
\hline $\mathrm{Al} / \mathrm{C}_{n} / \mathrm{Hg}, \mathrm{Al}$ or $\mathrm{Au}$ & 15 & conduction & 143,144 \\
\hline $\mathrm{Al} / \mathrm{C}_{n} / \mathrm{Mg}$ & 10 & conduction & 144 \\
\hline $\mathrm{Al} / \mathrm{C}_{n} / \mathrm{Al}$ & $10.5-15^{\mathrm{c}}$ & conduction, multilayer & 145 \\
\hline $\mathrm{Al} / \mathrm{C}_{n}$, dye $/ \mathrm{Al}$ & $3-5$ & photoconduction, multilayers & 146 \\
\hline dye $/ C_{n} / \mathrm{e}^{-}$acceptor & $\approx 3$ & fluorescence quenching & 147 \\
\hline $\mathrm{Al} / \mathrm{C}_{n} /$ anthracene & 4.5 & photoconduction & 148 \\
\hline $\mathrm{Al} / \mathrm{C}_{n} /$ chloranil & $\approx 8$ & photoconduction & 149 \\
\hline
\end{tabular}

a Many aromatic acceptors were used and gave an average value of $\beta=12 \mathrm{~nm}^{-1}$.

b TMPD denotes tetramethylphenylenediamine.

c Our estimate of the uncertainty in the slope of Fig. 2 in Ref. 145. A multilayered hopping formula wasused in Ref. 145 for the multilayer system.

or aluminum [145]. The results for $\beta$ were 15 $\mathrm{nm}^{-1}$, apart from that for magnesium $(\beta=10$ $\mathrm{nm}^{-1}$ ) and are included in Table IV. Analogous studies with multilayers [144] gave a value 10.5-15 (only a few points however), consistent with the monolayer studies if hopping between traps separated by the monolayers was assumed. Photoconduction experiments performed in multilayered structures dyes and fatty acids gave a smaller $\beta \approx 3-5 \mathrm{~nm}^{-1}$ [146]. A similarly small $\beta$ was obtained in fluorescence quenching experiments in which a layer of dye and a layer of acceptor were separated by a fatty-acid monolayer [147], and in photoconduction experiments in which a (semitransparent) aluminum layer was separated from an anthracene crystal [148] or a chloranil crystal [149] by a fatty acid monolayer. Thus, $\beta$ appears to be smaller when one of the reactants is electronically excited. (There is a smaller barrier in Fig. 2b then.)

(3) Particularly interesting are intramolecular electron-transfer rate measurements in which the separation distance is varied. In an experiment in which aromatic reactants are separated from each other by a steroid molecule, at an edge-to-edge separation distance of about $1.0 \mathrm{~nm}$ the electron transfer for the most exothermic reaction occurred in less than $0.5 \mathrm{~ns}$ [142]. Using a value for $k_{0}$ of $1 \cdot 10^{13} \mathrm{~s}^{-1}$ and $\Delta G^{*}=0$ yields $\beta<10 \mathrm{~nm}^{-1}$, while $k_{0}=1 \cdot 10^{14} \mathrm{~s}^{-1}$ yields $\beta<12 \mathrm{~nm}^{-1}$.

Summarizing these results we see that the value of the $\beta$ in Eqn. 37a for the aromatic systems in Table IV is about $11-12 \mathrm{~nm}^{-1}$, is higher when the transfer is from metals, and is less in both cases when photoexcited systems are involved. Incidentally, a flash photolysis study of electron transfer from an excited zinc porphyrin to a quinone ring bound a distance $r$ of $\approx 1.0 \mathrm{~nm}$ above the porphyrin plane yielded $k \approx 1 \cdot 10^{9} \mathrm{~s}^{-1}$ [150], while the maximum calculated $k$ assuming $\beta=12 \mathrm{~nm}^{-1}$, $r-r_{0}=0.7 \mathrm{~nm}$ (see below) and $k_{0}=1 \cdot 10^{13} \mathrm{~s}^{-1}$ (and $\Delta G^{*}=0$ ) is about $1 \cdot 10^{9} \mathrm{~s}^{-1}$. Clearly, further studies, including temperature dependences, are very desirable, and data for metalloprotein, polypeptide and other porphyrin systems are becoming available [38,151-156].

A rough interpretation of $\beta$ is in terms of electron tunneling through a square potential barrier, e.g., Ref. 143. (A more satisfactory model is discussed in Appendix IC.) Using a standard quantum-mechanical tunneling formula, $\beta$ equals $(2 / \hbar)\left(2 m V_{0}\right)^{1 / 2}$, where $m$ is the mass of the electron, $V_{0}$ the height of the potential barrier (Fig. 2) and $\hbar$ is $h / 2 \pi$. Values of $\beta$ of 11 and $15 \mathrm{~nm}^{-1}$ 
correspond to $V_{0}$ values of 1.1 and $2.1 \mathrm{eV}$, respectively. The $V_{0}$ is, in effect, a vertical ionization energy to the lowest unoccupied molecular orbital in the medium spanning the two redox centers, for the case where the nuclear configuration corresponds to the intersection region in Figs. 1 and 2. (Alternatively, it may be the corresponding quantity for a 'hole' [63].) The value of $14.4 \mathrm{~nm}^{-1}$ proposed for $\beta$ in Ref. [63] was based on an assumed barrier of $2 \mathrm{eV}$.

Strictly speaking, the pre-exponential factor $k_{0}$ in Eqn. 37a for a site-to-site electron transfer is also expected to have some polynomial dependence on $1 / r$, because of a geometrical factor appearing in the overlap of the electronic wavefunctions, see, e.g., Ref. 72. For the present purposes we shall regard this additional dependence on $r$ as approximately included in the exponential factor.

In addition to the electronic distance effect $\beta r$, there is another distance dependence which arises when the environment is a polar one, namely the increase of $\lambda$ with $r$, as in Eqn. 6: in the inverted region this additional effect causes the rate constant to increase initially with increasing $r$ and then, because of the exponential decrease of the electronic factor with $\beta r$, to decrease [158]. The dependence of $\lambda$ on $r$ can cause a wrong value of $\beta$ to be inferred from data on the distance dependence of intramolecular rate constants, unless allowance is made for it.

We next adapt Eqn. 33a to nonspherical molecules, such as the porphyrins occurring in biological systems. Structural information is normally given in terms of distances between atomic nuclei. However, a distance of particular interest for electron transfer is the distance between the closest atoms (of the two reactants) that are strongly coupled to their respective redox sites. When this distance is small enough $\kappa \nu$ equals some preassigned value, taken earlier as $1 \cdot 10^{13} \mathrm{~s}^{-1}$. Eqn. 33b can then be rewritten as

$\kappa \nu=1 \cdot 10^{13} \exp (-\beta d) \mathrm{s}^{-1}$,

where $d$ is equal to the actual separation of the centers of the two closest (in the case of aromatics) carbon atoms of the two reactants minus some amount to allow for the extension of the $\pi$-elec- tronic orbitals beyond the carbon nuclei. For concreteness we will use a value of $0.3 \mathrm{~nm}$ for the latter amount. (Thereby, if the frequency of motion along the reaction coordinate were about 1 . $10^{13} \mathrm{~s}^{-1}$, the reaction would be adiabatic $(\kappa=1)$ when these aromatic carbons are a distance $0.3 \mathrm{~nm}$ or less apart.) The value of $\kappa \nu$ also depends, of course, on the detailed geometry (e.g., Appendix IA), such as mutual orientation of the reactants, but for the present purpose we focus attention on the distance of closest approach.

\section{IVD. Work terms for bimolecular reactions involv- ing metalloproteins}

There have been a number of studies of the effect of ionic strength on the rates of electron transfer between metalloproteins and metal complexes [159-167]. The charges on the reactants are varied in these studies by using metal complexes of different charge types and/or by varying the charges on the protein through $\mathrm{pH}$ variations or by modifying the protein. The results are frequently interpreted by approximating the metalloprotein by a sphere and calculating its radius either from the partial specific volume of the protein in aqueous solution or from crystallographic data. In this model the charge of the protein is assumed to be uniformly distributed, and is calculated from the amino acid composition; this charge is then compared with the value calculated from the ionic strength dependence of the rate constants.

Use of Eqn. 25 for the electrostatic work required to bring together a metalloprotein and a metal complex (or two metalloproteins) yields Eqn. 38 for the ionic strength dependence of the rate constant ":

$$
\begin{aligned}
\ln k= & \ln \bar{k}-\frac{z_{1} z_{2} N \mathrm{e}^{2}}{2 D_{\mathrm{s}} \sigma_{12} R T}\left(\frac{\exp \left[-B\left(\sigma_{12}-\sigma_{1}\right) \sqrt{\mu}\right]}{1+B \sigma_{1} \sqrt{\mu}}\right. \\
& \left.+\frac{\exp \left[-B\left(\sigma_{12}-\sigma_{2}\right) \sqrt{\mu}\right]}{1+B \sigma_{2} \sqrt{\mu}}-2\right)
\end{aligned}
$$

\footnotetext{
" According to Eqns. 1-3 the ionic strength $\mu$ affects $k$ via the work terms $w^{\mathrm{r}}$ and $w^{\mathrm{p}}$ and via $\Delta G^{0}$. The latter depends on $\mu$ because properties of the medium do. When $\left|\Delta G^{0 \prime} / \lambda\right|$ is
} 
where $\vec{k}$ is the rate constant at infinite dilution, $\sigma_{12}$ is the center-to-center separation of the two reactants, and $\sigma_{1}$ and $\sigma_{2}$ are defined as for Eqn. 25. In using Eqn. 38 simplifying assumptions for $\sigma_{1}$ and $\sigma_{2}$ are often made: (i) $\sigma_{12}=\sigma_{1}+\sigma_{2}$ with $\sigma_{i}=a_{i}+0.15 \mathrm{~nm}$ [172] or (ii) $\sigma_{12}=\sigma_{1}+\sigma_{2}$ with $\sigma_{i}=a_{i}[163,169-171]$. The results obtained upon fitting the kinetic data to Eqn. 38 using the assumption (ii) and a least-squares routine in which $\vec{k}$ and the total charge on the protein are allowed to vary are summarized in Table V. Included in Table $\mathrm{V}$ are the protein charges calculated from the amino-acid composition. The results are seen to be in satisfactory agreement, except for the result reported for the reaction of stellacyanin with $\mathrm{Co}(\mathrm{phen})_{3}^{3+}$, which apparently exhibits no ionic strength dependence $\$$.

In another approach to ionic strength effects [172-175], it is assumed that the transition state of the reaction (denoted by $\mathrm{AB}^{\ddagger}$ ) can be regarded as a single body rather than a pair of reactants. The rate constant can then be written as in Eqn. 39 using transition state theory

$k=\bar{k} \gamma_{\mathrm{A}} \gamma_{\mathrm{B}} / \gamma_{\mathrm{AB}^{\ddagger}}$

where $\gamma_{\mathrm{A}}, \gamma_{\mathrm{B}}$ and $\gamma_{\mathrm{AB}^{*}}$ denote the activity coefficients of $A, B$ and $\mathrm{AB}^{\ddagger}$. The quantity $\gamma_{\mathrm{AB}^{\ddagger}}$ is assumed to be the activity coefficient of a sphere of radius $\sigma_{12}$ and total charge $\left(z_{1}+z_{2}\right)$. (Eqn. 39 was, in effect, obtained prior to transition state theory by Bronsted.) Expressing these activity coefficients in terms of Debye-Hückel theory, one obtains:

small, the dependence of $\ln k$ on $\mu$ is found from Eqns. 1-3 to be

$\ln k=\ln \bar{k}+\frac{1}{2 R T}\left(w^{\mathrm{r}}+w^{\mathrm{p}}\right)-\frac{1}{2} \ln \left(\gamma_{1}^{\mathrm{ox}} \gamma_{2}^{\mathrm{red}} / \gamma_{1}^{\mathrm{red}} \gamma_{2}^{\mathrm{ox}}\right)$

for the case of reaction 7 , where the $\gamma$ 's denote activity coefficients. For self-exchange reactions $w^{\mathrm{r}}$ equals $w^{\mathrm{p}}$, the activity coefficient ratio becomes unity, and use of Eqn. 25 then leads to Eqn. 38, where now $\sigma_{1}=\sigma_{2}$. An equation more general than Eqn. 38 and arising from the above expression for $\ln k$ is given in the Appendix as Eqn. 60 .

At the time this discrepancy was attributed, at least in part, to the presence of undefined amounts of carbohydrates in the preparation of the protein [159].

$$
\begin{aligned}
\ln k & =\ln \bar{k}-\frac{N e^{2} B \sqrt{\mu}}{2 D_{\mathrm{s}} R T} \\
& \times\left(\frac{z_{1}^{2}}{1+B \sigma_{1} \sqrt{\mu}}+\frac{z_{2}^{2}}{1+B \sigma_{2} \sqrt{\mu}}-\frac{\left(z_{1}+z_{2}\right)^{2}}{1+B \sigma_{12} \sqrt{\mu}}\right)
\end{aligned}
$$

At sufficiently low ionic strength Eqns. 38 and 40 both reduce to the same limiting form

$\ln k=\ln \bar{k}+\frac{z_{1} z_{2} N e^{2} B \sqrt{\mu}}{D_{s} R T}$

In Eqns. 1-3, and thereby in Eqn. 38 and in Eqn. 60 of the Appendix, the interaction of the reactants $A$ and $B$ is treated as outer-sphere-like, i.e., they do not coalesce. For Eqn. 40, on the other hand, A and B are bound closely enough (or, instead, one ion is very small relative to the other) that they have in effect coalesced to form a sphere. In general, Eqns. 38 and 40 yield fairly similar results $[170,172-175]$ : the difference between the expressions tends to become more marked at high ionic strengths, but Debye-Hückel-type theory also tends to break down under those conditions. The breakdown can be expected to be greatest for reactants of opposite charge because of specific ionic interactions [176], a point also noted later.

Eqns. 38 and 40 both assume that each reactant (and, in the case of Eqn. $40, \mathrm{AB}^{\ddagger}$ also) is spherical and has a uniform charge distribution. When the charge distribution on a reactant is asymmetric, as is the case for most proteins, the departure from spherical symmetry has been modelled by introducing dipole (and higher order) interactions. In recent applications, Eqn. 38 has been corrected by adding an ion-dipole interaction energy to the ion-ion interaction energy (Eqn. 25) [174-177], while Eqn. 40 has been corrected by using the Kirkwood expression for the activity coefficient of a charged dipole, which involves adding another term [172] to the Debye-Hückel term. Use of these expressions requires information about the dipole moment of the protein.

The dipole moment of cytochrome $c$ has been calculated to be $\approx 300$ Debye [173]. For ionic strengths below $0.1 \mathrm{M}$, this dipole moment hardly 


\section{TABLE V}

RESULTS OF CALCULATIONS OF THE CHARGE ON THE METALLOPROTEIN FROM THE IONIC STRENGTH DEPENDENCE OF THE ELECTRON-TRANSFER RATES

$\mathrm{HAs}^{-}$denotes the ascorbate anion. The references cited in the last column contain both sets of calculations.

\begin{tabular}{|c|c|c|c|c|c|}
\hline \multirow[t]{2}{*}{ Protein } & \multirow[t]{2}{*}{ Reagent } & \multirow[t]{2}{*}{$\mathrm{pH}$} & \multicolumn{2}{|l|}{ Charge } & \multirow[t]{2}{*}{ Refs. } \\
\hline & & & $\begin{array}{l}\text { calculated } \\
\text { from composition }\end{array}$ & $\begin{array}{l}\text { calculated } \\
\text { using Eqn. } 38\end{array}$ & \\
\hline Cytochrome $c$ (horse heart) & $\mathrm{HAs}^{-}$ & 7.2 & +8 & +7.2 & 163 \\
\hline Cytochrome $c$ (horse heart deriv.) & $\mathrm{HAs}^{-}$ & 7.2 & +6 & +5.2 & 163 \\
\hline Cytochrome $c-551$ (Pseudomonas aeriginosa) & $\mathrm{Co}(\text { phen })_{3}^{3+}$ & 7.0 & -3 & -4.3 & 164 \\
\hline Cytochrome $c-552$ (Euglena gracilis) & $\mathrm{HAs}^{-}$ & 7.2 & -8 & -7.3 & 163 \\
\hline Cytochrome $c-552$ & $\mathrm{Fe}(\mathrm{CN})_{6}^{4-}$ & 7.2 & -8 & -6.9 & 163 \\
\hline Cytochrome $c-552$ & $\mathrm{Fe}(\mathrm{CN})_{6}^{3-}$ & 7.2 & -9 & -8.3 & 163 \\
\hline Cytochrome $c$ (horse heart) & $\mathrm{Fe}(\mathrm{EDTA})^{2-}$ & 7.0 & +8 & +10 & 169,170 \\
\hline Cytochrome $c$ (Rhodospirillum rubrum) & $\mathrm{Fe}(\mathrm{EDTA})^{2-}$ & 7.0 & +3 & +2.3 & 169,170 \\
\hline Azurin (Pseudomonas aeriginosa) & $\mathrm{Fe}(\mathrm{EDTA})^{2-}$ & 6.8 & -1 & -1.1 & 171 \\
\hline Plastocyanin (Phaseolus vulgaris) & $\mathrm{Fe}(\mathrm{EDTA})^{2-}$ & 6.9 & $-8^{a}$ & -7.2 & 171 \\
\hline Stellacyanin ( Rhus vernicifera) & $\mathrm{Co}(\text { phen })_{3}^{3+}$ & 7.0 & +9 & 0.0 & 164 \\
\hline
\end{tabular}

a charge of -9 is calculated from more recent sequence studies (subsection VF).

makes any contribution to the ionic strength dependence of the rate, but at ionic strengths above $0.4 \mathrm{M}$ the ionic strength dependence is mainly determined by the dipole interaction $[174,175]$. (The ion-ion interaction varies as the $\sqrt{\mu}$ while the ion-dipole interaction varies as $\mu$.) If the charge on cytochrome $c$ is +8 , then the maximum correction, regardless of the ionic strength, to the charge on the protein was found to be \pm 3 , the exact value depending on the cosine of the angle between the protein dipole and the interaction site on the protein surface $[174,175]$.

In certain applications good agreement with the experimental ionic strength dependence has been obtained when the charge and radius of the presumed electron transfer site, rather than the total protein charge and radius, have been substituted into Eqns. 38 or 40 [166], or into more complex expressions [177]. In other applications the effective charge on the protein has been calculated from the ionic strength dependence of the binding of small anions or cations using the Bjerrum expression for the ion-pair formation constants. This procedure yielded good agreement between the effective charge and the charge of the binding site calculated from the protein structure [178], indicating that the binding constants in the systems studied are determined by the local rather than by the total protein charge (see discussion in Appen$\operatorname{dix}$ IB).

In some cases the good agreement with the experimental data found with one model or another may be fortuitous, particularly if the local charge has the same sign and happens to be of similar magnitude to the total protein charge. This is the case, for example, with horse-heart cytochrome $c$ - the total charge on the oxidized protein is +8 and the exposed heme edge (believed to be the electron transfer site, subsection VA) is surrounded by a ring of four positively charged amino acids. In the case of bean plastocyanin, the total charge of the oxidized protein is -8 , while the electron-transfer site for the reaction of plastocyanin with negatively charged complexes is believed to be uncharged; nevertheless, the ionic strength dependence of the reactions of plastocyanin with $\mathrm{Fe}(\mathrm{EDTA})^{2-}[171]$ and $\mathrm{Fe}(\mathrm{CN})_{6}^{4-}$ [166] is consistent with reaction with a negatively charged protein. Presumably in these systems the approach of the negatively charged complex is influenced by the protein charge nearest the electron-transfer site, which is a negatively charged region in the case of plastocyanin (discussed further in subsection VF), even though the presumed electron transfer site itself is uncharged. On the other hand, cytochrome $f$ is an example of a 
protein in which the charge on the binding site, as deduced from the effect of ionic strength on the reaction rate, is of opposite sign to the total protein charge [179].

As a general rule one expects that the total protein charge will dominate the electrostatic interactions at large reactant separations, with the local protein charge becoming more important as the separation distance decreases. The 'cross-over' distance will depend upon the detailed charge distribution of the protein and is difficult to calculate, although some progress in modeling the electrostatic interactions is being made $[177,180]$. (In some systems, hydrophobic interactions may have to be included.) The results of recent calculations $[177,180]$ for the reactions of a variety of $c$-type cytochromes support the view that a localized region of the cytochrome $c$ surface is primarily responsible for the observed electrostatic interactions. This site is identified with the exposed heme region, an interpretation that is consistent with the results of other studies. These are described in the next section.

\section{Applications to biological electron transfers}

\section{$V A$. Reactions of cytochrome $c$ with metal complexes}

The reactions of cytochrome $c$ with metal complexes form an interesting bridge between the inorganic electron-transfer reactions in solution and purely biological electron transfers. Accordingly, we begin this section with a discussion of cytochrome $c$ reactions and some of the relevant theory.
Cytochrome $c$ is probably the best characterized metalloprotein [181-183]. The heme group lies in a crevice of the protein and is covalently bonded to the protein by thioether bridges. The iron atom is situated in the plane of the porphyrin ring with the fifth and sixth coordination sites occupied by a ring nitrogen atom of His-18 and the sulfur atom of Met-80. An important feature of the cytochrome structure is that an edge of the porphyrin ring is located at the surface of the metalloprotein.

The rates of electron transfer between cytochrome $c$ and a large number of metal complexes have been studied. The complexes used are substitution inert, ensuring an outer-sphere mechanism, and have known self-exchange rates [95,96,184-187] and redox potentials (Table VI). Some of the complexes are positively charged, others negatively charged; some are hydrophobic (e.g., $\mathrm{Co}(\text { phen })_{3}^{3+/ 2+}$ ), and others hydrophilic (e.g., $\left.\mathrm{Ru}\left(\mathrm{NH}_{3}\right)_{6}^{3+/ 2+}\right)$. The benzimidazole- and the pyridine-pentaammineruthenium complexes provide a large, aromatic ligand for probing hydrophobic protein regions such as the heme crevice.

The rate constants for the reactions are summarized in Table VII, which also includes the values calculated from Eqn. 42, a common approximation to the cross-relation, Eqn. 10:

$k_{12} \approx\left(k_{11} k_{22} K_{12}\right)^{1 / 2}$.

The experimental value of $k_{11}=1.2 \cdot 10^{3} \mathrm{M}^{-1} \cdot \mathrm{s}^{-1}$ $(\mu=0.1 \mathrm{M}, \mathrm{pH} 7)$ for the rate constant of the ferrocytochrome-ferricytochrome exchange reaction [193] and the values of $k_{22}$, the exchange rate

TABLE VI

REDUCTION POTENTIALS, AVERAGE RADII, AND EXCHANGE RATE CONSTANTS OF METAL COMPLEXES AT $25^{\circ} \mathrm{C}$

The radii mentioned in column 3 are the average van der Waals radii of the reactants.

\begin{tabular}{|c|c|c|c|c|c|}
\hline & $\begin{array}{l}E^{\circ} \\
\text { (V vs. NHE) }\end{array}$ & $\begin{array}{l}\text { Radius } \\
\text { (nm) }\end{array}$ & $\begin{array}{l}\mu \\
(\mathrm{M})\end{array}$ & $\begin{array}{l}k_{\mathrm{ex}} \\
\left(\mathrm{M}^{-1} \cdot \mathrm{s}^{-1}\right)\end{array}$ & Refs. \\
\hline $\mathrm{Co}(\text { phen })_{3}^{3+/ 2+}$ & +0.36 & 0.70 & 0.1 & 45 & 167,95 \\
\hline $\mathrm{Ru}\left(\mathrm{NH}_{3}\right)_{6}^{3+/ 2+}$ & +0.07 & 0.33 & 0.1 & $3.2 \cdot 10^{3}$ & 96 \\
\hline $\mathrm{Ru}\left(\mathrm{NH}_{3}\right)_{5} \mathrm{py}^{3+/ 2+}$ & +0.32 & 0.38 & 0.1 & $1.1 \cdot 10^{5}$ & 168,184 \\
\hline $\mathrm{Ru}\left(\mathrm{NH}_{3}\right)_{5} \mathrm{BzIm}^{3+/ 2+}$ & +0.15 & 0.43 & $(0.1)$ & $5 \cdot 10^{4}$ & 185 \\
\hline $\mathrm{Fe}(\mathrm{EDTA})^{1-/ 2-}$ & +0.12 & 0.40 & $(0.1)$ & $3 \cdot 10^{4}$ & 186 \\
\hline
\end{tabular}


TABLE VII

RATE CONSTANTS FOR HORSE-HEART CYTOCHROME $c$ REACTIONS AT $25^{\circ} \mathrm{C}$ AND 0.1 M IONIC STRENGTH

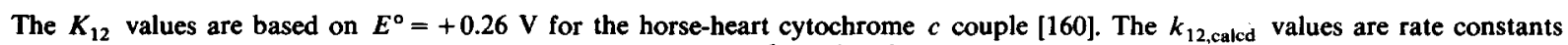
calculated in the present review from Eqn. 42, using $k_{11}=1.2 \cdot 10^{3} \mathrm{M}^{-1} \cdot \mathrm{s}^{-1}$ for the cytochrome $c$ self-exchange [193]. The calculated rate constants do not include the $W_{12}$ correction. $W_{12}$ is defined by Eqn. 24; the individual work terms were calculated from Eqn 25 .

\begin{tabular}{lcllll}
\hline Reagent & $K_{12}$ & $\begin{array}{l}k_{12, \text { obsd }} \\
\left(\mathrm{M}^{-1} \cdot \mathrm{s}^{-1}\right)\end{array}$ & $\begin{array}{l}k_{12, \text { calcd }} \\
\left(\mathrm{M}^{-1} \cdot \mathrm{s}^{-1}\right)\end{array}$ & $W_{12}$ & Ref. \\
\hline $\mathrm{Co}(\mathrm{phen})_{3}^{3+}$ & 49 & $1.5 \cdot 10^{3}$ & $1.6 \cdot 10^{3}$ & 1.1 & 188 \\
$\mathrm{Ru}\left(\mathrm{NH}_{3}\right)_{6}^{2+}$ & $1.6 \cdot 10^{3}$ & $3.8 \cdot 10^{4}$ & $7.8 \cdot 10^{4}$ & 1.9 & 189 \\
$\mathrm{Ru}^{3}\left(\mathrm{NH}_{3}\right)_{5} \mathrm{py}^{3+}$ & 8.5 & $9.6 \cdot 10^{3}$ & $3.4 \cdot 10^{4}$ & 1.6 & 190 \\
$\mathrm{Ru}\left(\mathrm{NH}_{3}\right)_{5} \mathrm{BzIm}^{2+}$ & 72 & $5.8 \cdot 10^{4}$ & $6.6 \cdot 10^{4}$ & 1.3 & 191 \\
$\mathrm{Fe}(\mathrm{edta})^{2-}$ & $2.3 \cdot 10^{2}$ & $2.6 \cdot 10^{4}$ & $9.1 \cdot 10^{4}$ & 9.1 & 192 \\
$\mathrm{Co}(\mathrm{ox})_{3}^{3-}$ & $1.7 \cdot 10^{5 \text { a.b }}$ & $5.5^{\mathrm{a}}$ & $7.0^{\mathrm{a} . \mathrm{c}}$ & $15^{\mathrm{a}}$ & 194 \\
\hline
\end{tabular}

Ionic strength, $0.5 \mathrm{M}$.

${ }^{b}$ Calculated using $E^{\circ}=+0.57 \mathrm{~V}$ for the $\mathrm{Co}(\mathrm{ox})_{3}^{3-14-}$ couple [194].

c Calculated using $k_{22}=2.4 \cdot 10^{-7} \mathrm{M}^{-1} \cdot \mathrm{s}^{-1}$ for the $\mathrm{Co}(\mathrm{ox})_{3}^{3-14-}$ exchange at $0.5 \mathrm{M}$ ionic strength. This value of $k_{22}$ was calculated from the rate constant for the oxidation of $\mathrm{Co}(\text { terpy })_{2}^{2+}$ by $\mathrm{Co}(\mathrm{ox})_{3}^{3-}$ reported in Ref. 190 using Eqn. 22.

constants for the metal complexes given in Table VI, were used. The $W_{12}$ correction, which was not used, is also given, and is seen in the table to be close to unity, except for the reaction of ferricytochrome $c$ with the negatively charged reactants $\mathrm{Fe}(\mathrm{EDTA})^{2-}$ and $\mathrm{Co}(\mathrm{ox})_{3}^{3-}$, for which $W_{12} \approx 9$ and $W_{12} \approx 15$, respectively.

The agreement of the observed and calculated exchange rates in Table VII is seen to be good (apart from that for the reactions with $\mathrm{Fe}(\mathrm{EDTA})^{2-}$ and $\mathrm{Co}(\mathrm{ox})_{3}^{3-}$ when $W_{12}$ is included). The simplest explanation is that cytochrome $c$ uses the same electron-transfer pathway with metal complexes as it does in its self-exchange reaction, and that this pathway is the exposed heme edge. Additional support for this mechanism has recently been obtained from protein modification studies [195]. One sees that there is no special rate enhancement with $\mathrm{Ru}\left(\mathrm{NH}_{3}\right)_{5} \mathrm{BzIm}^{2+}$ or $\mathrm{Ru}\left(\mathrm{NH}_{3}\right)_{5} \mathrm{py}^{3+}$ ascribable to penetration of the heme crevice by the $\pi$-conjugated ligand (Table VIII). This last result is in contrast to an earlier conclusion [190], which was based on the use of too small an exchange rate constant for the $\mathrm{Ru}\left(\mathrm{NH}_{3}\right)_{5} \mathrm{py}^{3+/ 2+}$ couple. The reaction of ferrocytochrome $c$ with $\mathrm{Co}(\mathrm{Ox})_{3}^{3-}$ is reasonably wellbehaved at the high ionic strength $(0.5 \mathrm{M})$ used for the studies. This is not the case for the reaction of cytochrome $c$ with ferro- and ferricyanide. There have been numerous studies of the latter reaction, and although rate saturation behavior has been reported at high concentrations of the complex, that interpretation is complicated by the need for corrections for specific ion interactions as well as for the reversibility of the reaction $[196,197]{ }^{n}$. At this time there appears to be no good example of rate saturation in the electron transfer reactions of cytochrome $c$ with metal complexes [197,198]. A report [199] that the $\mathrm{Fe}(\mathrm{CN})_{6}^{4-}$-ferricytochrome $c$ system exhibits an intervalence absorption band has also been shown to be erroneous: the observed band was due to Prussian-blue type impurities [201].

\section{$V B$. The cytochrome c self-exchange reaction}

The cross-reaction studies discussed above are clearly consistent with the cytochrome $c$ self-exchange rate of approx. $1 \cdot 10^{3} \mathrm{M}^{-1} \cdot \mathrm{s}^{-1}$ at $0.1 \mathrm{M}$ ionic strength determined by NMR [193]. In this section we interpret this rate constant in terms of the quantities introduced in Section II.

In terms of the model, the cytochrome $c$ self-exchange rate constant is given by (Appendix IB)

$$
k=S K_{\mathrm{A}} \kappa \nu \exp \frac{-\lambda}{4 R T}
$$

\footnotetext{
The redox potential and self-exchange rate of $\mathrm{Fe}(\mathrm{CN})_{6}^{3-/ 4-}$ couple is highly medium-dependent [187] and this couple is therefore not a good choice for cross-reaction studies.
} 

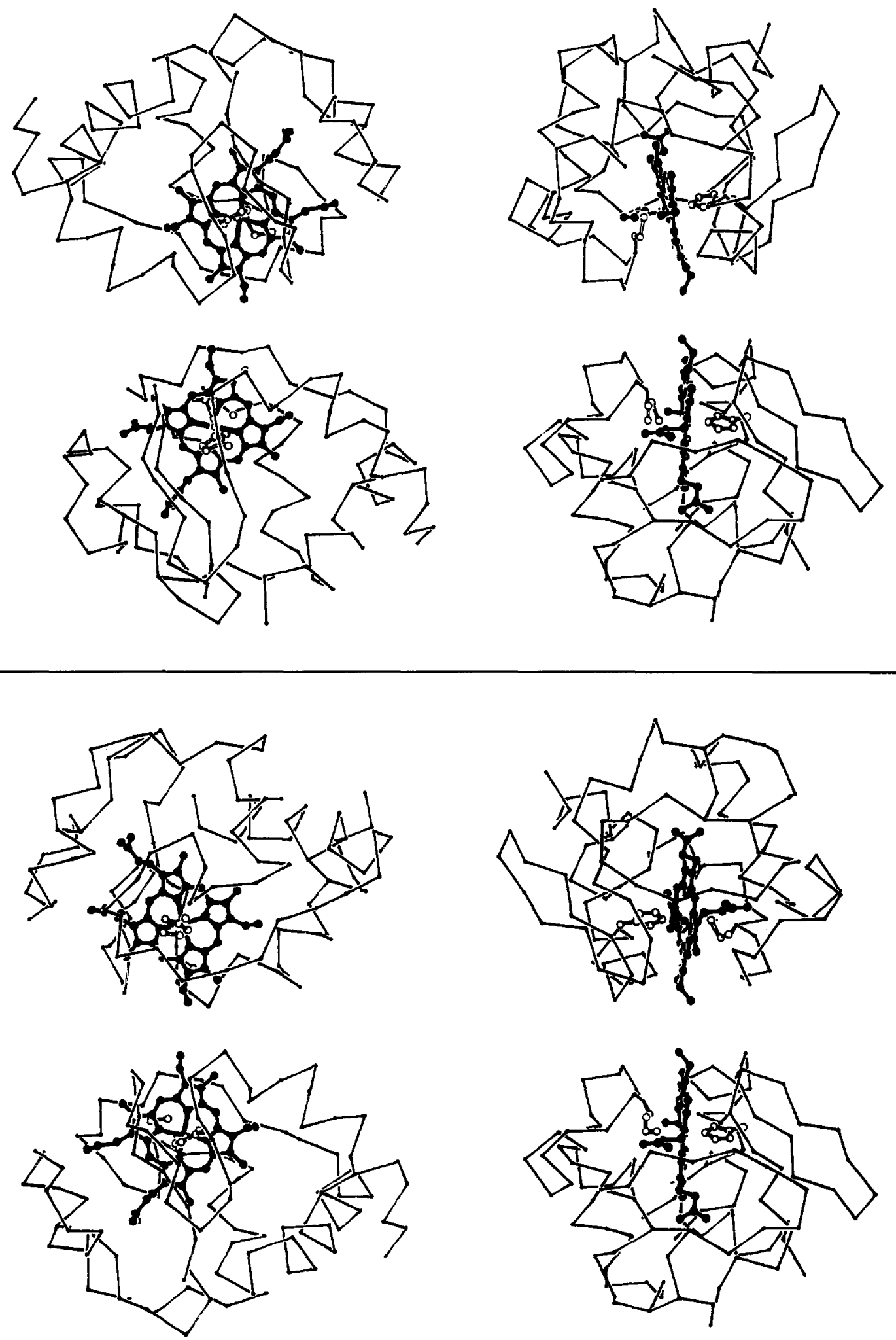

Fig. 9. Computer-generated complexes formed from two cytochrome $c$ molecules showing the coplanarity of the heme groups. Upper set: the two His-18 residues are cis relative to the Fe-Fe axis; lower set: the two His-18 residues are trans relative to the Fe-Fe axis. The figure was kindly provided by Dr. P.C. Weber of Genex Corporation. 
where $\lambda=\lambda_{\mathrm{i}}+\lambda_{\mathrm{o}}$. A steric factor $S$ has been introduced to allow for the fact that the cytochrome $c$ molecule is not spherically symmetric, at least so far as the angular dependence of the electron-transfer probability is concerned. The quantity $S K_{\mathrm{A}}$ is the equilibrium constant for formation of a precursor complex $\mathrm{A}-\mathrm{B}$ in which the active sites of $A$ and $B$ are in contact (Appendix IB).

We consider the various factors appearing in Eqn. (43): if it is assumed that the electron-transfer reactions of cytochrome $c$ proceed through the exposed, but recessed heme edge, then a lower limit for the steric factor for the exchange is equal to the square of the ratio of the surface area of the exposed heme edge to the surface area of the whole molecule [22]. Cytochrome $c$ is a prolate spheroid approximately $2.5 \times 2.5 \times 3.7 \mathrm{~nm}$ in size, corresponding to a surface area of $26 \mathrm{~nm}^{2}$. The effective area of the heme crevice is about $0.5 \times 1.8$ $\mathrm{nm}$ [200]; accordingly, the heme edge occupies about $3 \%$ of the surface of the molecule. We shall tentatively adopt a vlaue of 0.01 for $S$ in subsequent calculations . The $K_{\mathrm{A}}$ for this large system $(\sigma=2.84 \mathrm{~nm})$ is estimated with Eqns. 25 and 57 to be approx. $1 \mathrm{M}^{-1}$. As before, Eqn. $37 \mathrm{~b}$ of Section IVC is used for $\kappa \nu$.

Mixing of the $t_{2 \mathrm{~g}}$-orbitals of the Fe center in cytochrome $c$ with the $\pi^{*}$-orbitals of the porphyrin ring effectively extends the metal d-electron density to the porphyrin edge, so that facile electron transport to and from the iron center via the exposed heme edge should be possible. However, this does not mean that the exchange reaction will be adiabatic $\$$, i.e., that $\kappa \approx 1$. The 'exposed' edge of the heme group of cytochrome $c$ is surrounded by folds in the polypeptide chain: these folds may

\footnotetext{
I In general, it is necessary to integrate $k(r)$ over all mutual orientations and distances of the reacting pair. We adopt a simplified model in which the electron transfer through the remainder of the protein molecule may be neglected in comparison with the rate through the heme edge. Because of electron transfer from other angles the actual $S$ is expected to be closer to unity than the square of the ratio of effective areas $(0.03)^{2}$ and we have adopted the value of 0.01 .

Nor does the fact that the cross-reactions satisfy Eqn. 42 require that the exchange reaction be adiabatic; Eqn. 42 will still be satisfied provided $\kappa_{12} \approx\left(\kappa_{11} \kappa_{22}\right)^{1 / 2}$ or, more generally, provided that $S_{12} \kappa_{12} \approx\left(S_{11} S_{22} \kappa_{11} \kappa_{22}\right)^{1 / 2}$ [22].
}

prevent the close (edge-to-edge) approach of the hemes required for $\kappa \approx 1$. Computer modeling (Weber, P.C. (1984) personal communication to Sutin, N) of the approach of two cytochrome $c$ molecules reveals that the heme groups are able to approach closest in a co-planar configuration. Two such complexes, in which the His-18 residues are cis and trans, respectively, relative to the $\mathrm{Fe}-\mathrm{Fe}$ axis, are shown in Fig. 9. In these complexes the heme edge-to-edge (carbon-carbon) separation is $0.94 \mathrm{~nm}$ and the iron-iron distance is $1.77 \mathrm{~nm}$. (Interestingly, this value is close to that found for two similarly docked cytochromes, $1.6 \mathrm{~nm}$, in an independent study - an X-ray structure determination of a photosynthetic reaction center discussed in subsection VD.) Although there are more short heme-heme distances in the cis complex, this advantage is offset by the lower electrostatic repulsion in the trans complex. By movement of the side chains of the surface amino acids the separation of the heme groups can be decreased from $0.94 \mathrm{~nm}$ to approx. $0.9 \mathrm{~nm}$, but cannot readily be reduced much further (Weber, P.C. (1984) personal communication to Sutin, N.). For purposes of calculation, we use the former value for the heme edge-to-edge (carbon-to-carbon) separation in the cytochrome $c$ exchange, and use the $0.3 \mathrm{~nm}$ correction discussed in subsection IVB to obtain an electron-transfer distance $d$ of 0.64 $\mathrm{nm}$. With this value of $d$ and $\beta=12 \mathrm{~nm}^{-1}$, a value of $\kappa \nu \approx 5 \cdot 10^{9} \mathrm{~s}^{-1}$ would be estimated ".

Next we consider the values of $\lambda_{i}$ and $\lambda_{0}$. $X$-ray crystallographic studies $(0.18 \mathrm{~nm}$ resolution) indicate minimal differences between the structures of the heme group in oxidized and reduced cytochrome $c[181,182]$. A crystal of ferricytochrome $c$ (but not of ferrocytochrome $c$ ) contains two molecules per unit cell: the average $\mathrm{Fe}$ to

\footnotetext{
T Alternative electron-transfer pathways are not considered. For example, mediation by the sulfur atoms of Cys-14 and Cys-17, which are covalently bonded to the exposed heme edge, could be important [202]. In this regard the sulfur atom of Cys-17 is particularly well-situated (Fig. 9) and extended Hückel calculations (Hanson, L.R. (1984) personal communication to Sutin, N.) indicate that the Fe electron density on the cysteinyl sulfurs may be comparable to that on the heme-edge carbon atoms. Mediation by the sulfur would decrease the effective $d$ and increase $\kappa \nu$.
} 
pyrrole- $\mathrm{N}$ distances in the two molecules are 0.208 \pm 0.011 and $0.203 \pm 0.011 \mathrm{~nm}$, while in ferrocytochrome $c$ this distance is $0.206 \pm 0.007 \mathrm{~nm}$; the Fe to His- $18 \mathrm{~N}$ distance is $0.196 \pm 0.011$ and $0.204 \pm$ $0.011 \mathrm{~nm}$ in the two ferricytochrome $c$ molecules and $0.197 \pm 0.007 \mathrm{~nm}$ in the reduced molecule, while the Fe to Met-80S distance is $0.228 \pm 0.005$ $\mathrm{nm}$ and $0.226 \pm 0.005 \mathrm{~nm}$ in the two oxidized molecules and $0.232 \pm 0.003 \mathrm{~nm}$ in the reduced molecule [182]. Although the iron-ligand distances in the two oxidation states appear to be similar the uncertainty is too large to permit a good estimae of $\lambda_{i} / 4$, the reorganization energy arising from changes in the metal ligand bond distances. A small value of $\lambda_{i} / 4$ (approx. $4-8 \mathrm{~kJ} \cdot \mathrm{mol}^{-1}$ ) is suggested by the rapid self-exchange rates of free (i.e., without bound protein) low-spin ${ }^{\S}$ hemes in solution, and is not inconsistent with the $\mathrm{X}$-ray data. This value of $\lambda_{i}$ for a pair of cytochromes is also consistent with the Stokes shift $\left(8 \mathrm{~kJ} \cdot \mathrm{mol}^{-1}\right)$ [204] for the low-frequency modes $\left(200-500 \mathrm{~cm}^{-1}\right.$ range) that are coupled to the heme $\pi-\pi^{*}$ transition in a single ferrocytochrome $c$. (If these lowfrequency modes provided the only contribution to $\lambda_{i}$ for the self-exchange, such a Stokes shift would yield $\lambda_{\mathrm{i}}=8 \times 2 \mathrm{~kJ} \cdot \mathrm{mol}^{-1}$ ).

For the metalloprotein reactions there are, in general, two contributions to $\lambda_{\mathrm{o}}$, one arising from the change in polarization (orientations of protein dipoles) of the polypeptide and the second from the change in polarization of the surrounding solvent. In general, the relative contributions to $\lambda_{\mathrm{o}}$ from these two sources will depend upon the nature of the particular metalloprotein. For cytochrome $c$, the contributions of the reorientation of the protein dipoles and of the surrounding water molecules to $\lambda_{o}$ (for the pair of cytochrome reactants) have been calculated from a microscopic model to be 10 and $12 \mathrm{~kJ} \cdot \mathrm{mol}^{-1}$, respectively, and $\lambda_{i}$ was estimated to be $4 \mathrm{~kJ} \cdot \mathrm{mol}^{-1}$ [137]. The resulting value of $6.5 \mathrm{~kJ} \cdot \mathrm{mol}^{-1}$ for $\lambda / 4$ seems too low. An alternative approach would be to assume that the protein/water surrounding the heme can be treated as a continuous dielectric with $D_{\mathrm{s}}=10$ and $n^{2}=2$. If Eqn. 6 is then used with $a$, the

\footnotetext{
The value of $\lambda_{i} / 4$ is likely to be larger for high-spin than for low-spin hemes because of the larger changes in metal to axial-ligand distances expected for high-spin systems.
}

radius of the heme, equal to $0.5 \mathrm{~nm}$ and $r$, the iron-to-iron distance, equal to $1.8 \mathrm{~nm}, \lambda_{\mathrm{o}} / 4 \mathrm{be}$ comes $20 \mathrm{~kJ} \cdot \mathrm{mol}^{-1}$. A total value of $25 \mathrm{~kJ} \cdot \mathrm{mol}^{-1}$ for $\lambda / 4$ for the Cyt $c$-Cyt $c$ self-exchange is consistent with the activation energy of $29 \pm 4$

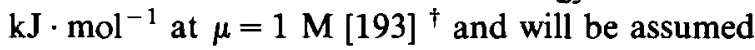
here, together with an $S$ of 0.01 for that reaction and $\beta=12 \mathrm{~nm}^{-1}$. Considering the complexities of the systems and the current uncertainties in their molecular parameters, a factor of ten difference in calculated and observed rates (which is equivalent to a change of $5.8 \mathrm{~kJ} \cdot \mathrm{mol}^{-1}$ in the free-energy barrier) can be regarded as good agreement.

The particular data which will be considered using the above values " of $\beta, S$ and $\lambda$ are: (a) the self-exchange rate constant of the cytochrome $c$ system; (b) the self-exchange for the protein-free hemes; (c) the bimolecular electron transfer between ferricytochrome $c$ and $\mathrm{Ru}\left(\mathrm{NH}_{3}\right)_{5} \mathrm{His}^{2+}$; (d) the intramolecular $\left(\mathrm{NH}_{3}\right)_{5} \mathrm{RuHis}-\mathrm{Cyt} c$ electron transfer, its small activation energy and its very negative entropy of activation; (e) the maximum bimolecular rate constant for electron transfer from Cyt $c$ to various metal complexes; (f) the cytochrome $c$-chlorophyll dimer electron transfer in a photosynthetic reaction center; $(\mathrm{g})$ the relative rates of the Cyt $c-\mathrm{Cyt} b_{5}$ and Cyt $c$-Cyt $c$ reactions; and $(\mathrm{h})$ electron transfer between cytochrome $c$ and a number of other metalloproteins. The bulk of these results and several others are collected later in Table VIII.

With the above values for $S\left(\approx 10^{-2}\right), K_{\mathrm{A}}(\approx 1$ $\left.\mathrm{M}^{-1}\right), \quad \kappa \nu\left(\approx 5 \cdot 10^{9} \mathrm{~s}^{-1}\right)$ and $\lambda / 4(\approx 25 \mathrm{~kJ}$. $\left.\mathrm{mol}^{-1}\right)$, the self-exchange rate constant for cytochrome $c$ is estimated from Eqn. 43 to be $10^{-2}$.

\footnotetext{
† The activation energy at $\mu=0.1 \mathrm{M}$ is twice as large [193]; perhaps there is a large temperature-dependent salt effect at low ionic strengths. Debye-Hückel type expressions appear to be more valid for calculating free energy changes (e.g., activity coefficients or work terms) than for calculating their derivatives, such as entropies or energies (cf. results in Ref. 76).

T Predicted results in Section V, such as the Cyt $c-C y t c$ self-exchange rate or the cross-reaction rate at small $\left|\Delta G^{0 \prime} / \lambda\right|$ do not depend on the individual values of $S$ and $\lambda\left(S_{11}\right.$ and $\left.\lambda_{11}\right)$, but only on the combination $S_{11} \exp \left(-\lambda_{11} / 4 R T\right)$, [cf. Eqn. 43]. Predicted results such as an intramolecular electron transfer $k$ or its activation energy depend on $\lambda_{11} / 2$, but not on $S_{11}$, while the predicted $k$ in the barrierless regime depends on $S_{1}^{1}{ }^{2}$, but not on $\lambda_{11}$.
} 
$5 \cdot 10^{9} \mathrm{e}^{-10}$ i.e., approx. $2 \cdot 10^{3} \mathrm{M}^{-1} \cdot \mathrm{s}^{-1}$. The experimental value is $1.2 \cdot 10^{3} \mathrm{M}^{-1} \cdot \mathrm{s}^{-1}$ [193].

The rate constant $k$ for heme-heme self-exchange is $1 \cdot 10^{6}-1 \cdot 10^{10} \mathrm{M}^{-1} \cdot \mathrm{s}^{-1}$ depending on the nature of the axial ligands (through their effect on $\lambda_{i}$ and $\lambda_{o}$ ) and the medium [205]. The value of $\lambda_{\mathrm{o}} / 4$ for heme exchange will depend on the nature of the solvent, but is expected to be small because of the relatively large size of the heme group. The factor of more than $10^{4}$ difference in the cytochrome $c$ and free heme self-exchange rates is then primarily due to the extra separation distance between the hemes in the protein-protein case ${ }^{8}$. The large separation was estimated above the introduce a factor of $5 \cdot 10^{-4}$ into the $k$ for the Cyt $c-$ Cyt $c$ exchange. In general, the value of $k$ for a hemeprotein self-exchange will be sensitive to the degree of exposure of the heme and, all other factors being equal, changing the exposure of the heme by changing the protein conformation or the number of amino acids is likely to dramatically affect the exchange rate of the cytochrome. For example, Pseudomonas aeruginosa cytochrome $c$ 551 has only 82 amino acid residues compared with the 104 residues of horse-heart cytochrome $c$, and has a self-exchange rate of $1.2 \cdot 10^{7} \mathrm{M}^{-1} \cdot \mathrm{s}^{-1}$ [206], instead of $1.2 \cdot 10^{3} \mathrm{M}^{-1} \cdot \mathrm{s}^{-1}$. A part of this difference may be due to the smaller size of Cyt $c-551$, which makes the hemes more accessible, and part may be due to a difference in work terms, e.g., the electrostatic repulsion in the Cyt c-551 self-exchange is smaller (cf. Table $\mathrm{V}$ for the ionic charges).

\section{VC. Intramolecular electron transfer}

Rate constants calculated with the above parameters can be compared with data on the intramolecular electron transfer between ferricytochrome $c$ and a bound $\mathrm{Ru}\left(\mathrm{NH}_{3}\right)_{5}^{2+}$ residue.

We begin with the value of the bimolecular rate constant for reduction of ferricytochrome $c$ by $\mathrm{Ru}\left(\mathrm{NH}_{3}\right)_{5} \mathrm{His}^{2+}, 9 \cdot 10^{4} \mathrm{M}^{-1} \cdot \mathrm{s}^{-1}$ [207]. When

\footnotetext{
The respective values of $S \sigma^{2}$ also enter. Because of orientation requirements the $S$ for the free heme system may be less than unity. Consequently, we estimate that $S \sigma^{2}$ is $10^{-2}(3)^{2}$ $\mathrm{nm}^{2}$ and less than $1 \mathrm{~nm}^{2}$ for the protein-bound and proteinfree heme systems, respectively.
}

$\left|\Delta G^{0 \prime}\right| \lambda$ is small, the theoretical rate constant for this reaction is given by (Appendix IB):

$k \cong S K_{\mathrm{A}} \kappa \nu \exp \left(\frac{-\lambda}{4 R T}-\frac{\Delta G^{o \prime}}{2 R T}\right)$,

where $k$ is measured in $\mathrm{M}^{-1} \cdot \mathrm{s}^{-1}$ and where $S_{\kappa \nu}$ $=2 \cdot 10^{10} \mathrm{~s}^{-1}$, based on $S=(0.01)^{1 / 2}$ and $\kappa \nu$ calculated from $1 \cdot 10^{13} \exp (-\beta d)$ with $\beta=12$ $\mathrm{nm}^{-1}$ and an electron-transfer distance $\mathrm{d} \approx 0.32$ $\mathrm{nm}$. (This distance is half the value used in subsection VB for the cytochrome $c$ self-exchange; the electron is assumed to be delocalized to the edge of the electronic cloud of the hydrogen atoms on the periphery of the $\mathrm{Ru}\left(\mathrm{NH}_{3}\right)_{5}$ His complex.) For $\sigma=1.8 \mathrm{~nm}$ and $\mu=0.1 \mathrm{M}, K_{\mathrm{A}}$ is about $0.4 \mathrm{M}^{-1}$ (including only electrostatic contributions to $w$ ). Using the parameters for the cytochrome $c$ exchange estimated above and $\mathrm{Ru}\left(\mathrm{NH}_{3}\right)_{5} \mathrm{py}^{3+/ 2+}$ [96] as a model for the $\mathrm{Ru}\left(\mathrm{NH}_{3}\right)_{5} \mathrm{His}^{3+/ 2+} \mathrm{ex}$ change, $\lambda / 4$, and $\Delta G^{0} / 2$ are calculated to be 27.2 and $-8.4 \mathrm{~kJ} \cdot \mathrm{mol}^{-1}$, respectively $\$$. Substituting these values into Eqn. 44 gives $k_{\text {calc }} \approx\left(2 \cdot 10^{10}\right)$ (0.4) $\mathrm{e}^{-7.5}$, i.e., $4 \cdot 10^{6} \mathrm{M}^{-1} \cdot \mathrm{s}^{-1}$. The observed value is $9 \cdot 10^{4} \mathrm{M}^{-1} \cdot \mathrm{s}^{-1}$ [207].

This experimental rate constant of $9 \cdot 10^{4} \mathrm{M}^{-1}$ $\cdot \mathrm{s}^{-1}$ may be converted to a first-order constant $k_{1}$ for electron transfer at the heme edge by setting $k_{1}$ equal to $k / S K_{\mathrm{A}}$ (Appendix IB). With $S \approx(0.01)^{1 / 2}$ and $K_{\mathrm{A}}=0.4 \mathrm{M}^{-1}$ for the cited $\sigma$ (cf. Eqn. 57 of Appendix I with $w^{\mathrm{r}}$, assumed for simplicity to be nonelectrostatic, given by Eqn. 25) the first-order rate constant $k_{1}$ equals $2 \cdot 10^{6} \mathrm{~s}^{-1}$. This $k_{1}$ is introduced into Eqn. 45 below.

By the use of imaginative synthetic techniques, a $\mathrm{Ru}\left(\mathrm{NH}_{3}\right)_{5}$-residue has been covalently attached to His-33 of ferricytochrome $c$ and the intramolecular electron-transfer rate from the ruthenium(II) to the heme iron(III) measured [151-157]. The closest distance from the imidazole group of His-33 to the imidazole group that is coordinated to the

\footnotetext{
The value of $\lambda$ for the cross-reaction was taken equal to the mean of the $\lambda$ values for the corresponding exchange reactions. For the Cyt $c-C y t c$ exchange the value of $\lambda / 4 \approx 25$ $\mathrm{kJ} \cdot \mathrm{mol}^{-1}$ mentioned earlier was used, while $\lambda / 4$ for the $\mathrm{Ru}\left(\mathrm{NH}_{3}\right)_{5} \mathrm{py}^{3+/ 2+}$ couple is about $29 \mathrm{~kJ} \cdot \mathrm{mol}^{-1}$ [96]. The $\sigma=1.8 \mathrm{~nm}$ is the sum of the radii of the two reactants $(1.42$ $\mathrm{nm}$ plus $0.38 \mathrm{~nm}) . \Delta G^{0}$, defined in subsection IIA, depends on ionic strength, as do the $w$ 's.
} 
heme iron is $1.18 \mathrm{~nm}$ [155] and the measured intramolecular rate constant is $30 \pm 3 \mathrm{~s}^{-1}$ by flash photolysis [155] and $53 \pm 2 s^{-1}$ by pulse radiolysis [156] techniques. The rate constant may be compared with $2 \cdot 10^{6} \mathrm{~s}^{-1}$ estimated above for 'intramolecular' electron transfer at the heme edge (the driving force, $-\Delta G^{0}$, for the two electron transfers is similar). It is seen that the adjacent heme-edge pathway is approx. $10^{5}$ times more favorable than the remote His-33 pathway, which involves electron transfer through the protein.

Assuming that $\lambda, \Delta G^{0}$, and $\beta$ are the same for the adjacent and remote pathways, the rate constant $k$ for the remote pathway can be estimated from $k_{1}$ and the change in separation distance:

$k=k_{1} \exp (-\beta \Delta d)$

where $k$ is measured in $\mathrm{s}^{-1}$ and $k_{1} \approx 2 \cdot 10^{6} \mathrm{~s}^{-1}$. The difference in the electron-transfer distances for the adjacent and remote pathways $\Delta d \approx 0.7$ $\mathrm{nm}^{\S}$ and $\beta=12 \mathrm{~nm}^{-1}$. Substitution into Eqn. 45 gives $k_{\text {calc }} \approx 500 \mathrm{~s}^{-1}$, which is to be compared with the observed value of $30-53 \mathrm{~s}^{-1}$.

In addition to the rate constant, the temperature dependence of the intramolecular ferricytochrome $c$ to $\mathrm{Ru}\left(\mathrm{NH}_{3}\right)_{5} \mathrm{His}^{2+}$ rate was also measured. The flash photolysis results give $\Delta H^{*} \leq 6.3$ $\mathrm{kJ} \cdot \mathrm{mol}^{-1}$ and $\Delta S^{\dagger}=-200 \pm 8 \mathrm{~J} \cdot \mathrm{deg}^{-1} \cdot \mathrm{mol}^{-1}$ [155], while the pulse radiolysis studies yield $\Delta H^{*}$. $=14.6 \pm 0.8 \mathrm{~kJ} \cdot \mathrm{mol}^{-1}$ and $\Delta S^{\dagger}=-163 \pm 4 \mathrm{~J} \cdot$." $\mathrm{deg}^{-1} \cdot \mathrm{mol}^{-1}[156]$. Although there is some disagreement, $\Delta H^{*}$ for both studies is relatively small while the entropy of activation $\Delta S^{\dagger}$ is large and negative. The low value of $\Delta H^{*}$ derives, we believe, largely from the negative $\Delta H^{0}$ for the reaction: The reorganization enthalpy at the separation distance $r$ is related to the standard enthalpy change of the reaction $\Delta H^{0^{\prime}}$ at that separation distance in the prevailing medium by $[60]^{\sharp}$ :

$\Delta H_{12}^{*}=\frac{\Delta H_{11}^{*}}{2}+\frac{\Delta H_{22}^{*}}{2}+\frac{\Delta H^{0^{\prime}}}{2}$,

8 This value assumes the shortest path for electron transfer to be a linear one. Another possibility is a more tortuous path along a polypeptide backbone.

\# Eqn. 46 follows from Eqn. 35 when $\left|\Delta G^{0 \prime} / \lambda\right|$ is small compared to unity. In the present case $\Delta G^{0}$ is $-17 \mathrm{~kJ} \cdot \mathrm{mol}^{-1}$ and $\lambda$ is $108 \mathrm{~kJ} \cdot \mathrm{mol}^{-1}$. where $\Delta H_{11}^{*}$ and $\Delta H_{22}^{*}$, the reorganization enthalpies of the component exchange reactions, are approximately equal to $\lambda_{11} / 4$ and $\lambda_{22} / 4$, respectively. From the value of $\Delta H^{0}=-49.8 \mathrm{~kJ} \cdot \mathrm{mol}^{-1}$ $[155]^{\#}$ and the values used earlier for the $\lambda$ 's $\left(\Delta H_{11}^{*}=25 \mathrm{~kJ} \cdot \mathrm{mol}^{-1}\right.$ and $\Delta H_{22}^{*}=29 \mathrm{~kJ} \cdot \mathrm{mol}^{-1}$ [96]), one finds $\Delta H_{12}^{*}=2.1 \mathrm{~kJ} \cdot \mathrm{mol}^{-1}$, consistent with the small observed $[155,156]$ value. A similar analysis for the corresponding azurin$\mathrm{Ru}\left(\mathrm{NH}_{3}\right)_{5} \mathrm{His}^{2+}$ molecule has just been published [208]. Finally, in terms of the present model, the entropy of activation $\Delta S^{\dagger}$ for the intramolecular electron transfer can be interpreted using the expression, obtained from Eqns. 36c, 36d and 37b:

$\Delta S_{12}^{\dagger} \cong \frac{\Delta S^{0^{\prime}}}{2}-R \beta d$,

neglecting the $\Delta G^{0^{\prime}} / \lambda$ term. The value of $\Delta S^{0} / 2$ is $-52.3 \mathrm{~J} \cdot \mathrm{deg}^{-1} \cdot \mathrm{mol}^{-1}[155]^{\pi}$ and $-R \beta d$ equals $-100 \mathrm{~J} \cdot \mathrm{deg}^{-1} \cdot \mathrm{mol}^{-1}$ (calculated, as above with $\beta=12 \mathrm{~nm}^{-1}$ and $d=1.0 \mathrm{~nm}$ ). One thus finds $-152.8 \mathrm{~J} \cdot \mathrm{deg}^{-1} \cdot \mathrm{mol}^{-1}$ for the calculated $\Delta S_{12}^{\dagger}$, while the observed value is -163 [156] or -200 $[155] \mathrm{J} \cdot \mathrm{mol}^{-1} \cdot \mathrm{deg}^{-1}$.

It is also useful to examine experimental data on the maximum value of the rate constant observed in the barrierless $\left(-\Delta G^{0^{\prime}} \approx \lambda\right)$ regime for the electron transfer from Cyt $c$ to various reactants and to compare this rate constant with calculated values. For the reaction of ferricytochrome $c$ with the charge-transfer excited state of $\mathrm{Ru}(\mathrm{bpy})_{3}^{2+},\left(\Delta G^{0} \approx-1.1 \mathrm{~V}\right)$ the observed rate constant is $2.5 \cdot 10^{8} \mathrm{M}^{-1} \cdot \mathrm{s}^{-1}$ at $0.1 \mathrm{M}$ ionic strength [209]. For the reaction of ferrocytochrome $c$ with $\mathrm{Ru}(\mathrm{bpy})_{3}^{3+}, \mathrm{Ru}(\mathrm{phen})_{3}^{3+}, \mathrm{Os}(\mathrm{bpy})_{3}^{3+}$ and $\mathrm{Os}(\mathrm{phen})_{3}^{3+}$, all reactions having large negative $\Delta G^{0^{\prime}}$ 's, the rate constants are $1.2,0.65,1.2$ and 0.76 , respectively, in units of $10^{8} \mathrm{M}^{-1} \cdot \mathrm{s}^{-1}$. The maximum value of the 'activation-controlled rate constant', $S K_{\mathrm{A}} \kappa \nu$ (Appendix IB) can be calculated from the parameters estimated earlier to be approx. $8 \cdot 10^{9} \mathrm{M}^{-1} \cdot \mathrm{s}^{-1}$. The diffusion-controlled rate constant ( $k_{\mathrm{D}}$ in Eqn. 53b of Appendix IA) for the reaction of the cytochrome $c$ 's with large +3

\footnotetext{
The difference between $\Delta H^{0}$ and the $\Delta H^{0}$ in Eqn. 46 is negligible in the present system for the remote pathway, as is that between $\Delta S^{0}$ and the $\Delta S^{0 \prime}$ in Eqn. 47.
} 
TABLE VIII

ABSOLUTE RATE CONSTANTS OR ACTIVATION PARAMETERS FOR SEVERAL CYTOCHROME $c$ REACTIONS

\begin{tabular}{|c|c|c|c|c|c|}
\hline Reaction & $\begin{array}{l}\Delta G^{0} \\
\left(\mathrm{~kJ} \cdot \mathrm{mol}^{-1}\right)\end{array}$ & Parameter & Observed & Calculated & Subsection \\
\hline Cyt $c^{I I}+\mathrm{Cyt} c^{\mathrm{III}}$ & $\begin{array}{l}0 \\
0\end{array}$ & $\begin{array}{l}E_{\mathrm{a}}(\mu=1 \mathrm{M})\left(\mathrm{kJ} \cdot \mathrm{mol}^{-1}\right) \\
k(\mu=0.1 \mathrm{M})\left(\mathrm{M}^{-1} \cdot \mathrm{s}^{-1}\right)\end{array}$ & $\begin{array}{l}29 \pm 4 \\
1 \cdot 10^{3}\end{array}$ & $\begin{array}{l}25^{a} \\
2 \cdot 10^{3}\end{array}$ & $\begin{array}{l}\text { VB } \\
\text { VB }\end{array}$ \\
\hline $\mathrm{Cyt} c^{\mathrm{III}}+\mathrm{Ru}\left(\mathrm{NH}_{3}\right)_{5} \mathrm{His}^{2+}$ & -17 & $k\left(\mathrm{M}^{-1} \cdot \mathrm{s}^{-1}\right)$ & $9 \cdot 10^{4}$ & $4 \cdot 10^{6}$ & VC \\
\hline 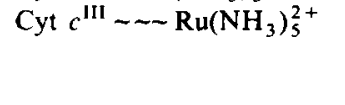 & $\begin{array}{l}-17 \\
-50(\leqslant 4)^{b} \\
-105(-202)^{c}\end{array}$ & $\begin{array}{l}k\left(\mathrm{~s}^{-1}\right) \\
\Delta H^{*}\left(\mathrm{~kJ} \cdot \mathrm{mol}^{-1}\right) \\
\Delta S^{*}\left(\mathrm{~J} \cdot \mathrm{deg}^{-1} \mathrm{~mol}^{-1}\right)\end{array}$ & $\begin{array}{l}30-50 \\
146 \\
-163\end{array}$ & $\begin{array}{l}500 \\
2 \\
-152\end{array}$ & $\begin{array}{l}\mathrm{VC} \\
\mathrm{VC} \\
\mathrm{VC}\end{array}$ \\
\hline $\begin{array}{l}\text { Cyt } c^{\mathrm{II}}+\mathrm{ML}_{3}^{3+\mathrm{d}} \\
\text { Cyt } c^{\mathrm{II}} \sim \sim \mathrm{BChl}_{2}^{+}\end{array}$ & $\begin{array}{l}-59 \text { to }-84 \\
\approx-16\end{array}$ & $\begin{array}{l}k_{\max }\left(\mathrm{M}^{-1} \cdot \mathrm{s}^{-1}\right) \\
k\left(\mathrm{~s}^{-1}\right)\end{array}$ & $\begin{array}{l}\approx 10^{8} \\
4 \cdot 10^{6}\end{array}$ & $\begin{array}{l}3 \cdot 10^{9} \mathrm{e} \\
4 \cdot 10^{7}\end{array}$ & $\begin{array}{l}\text { VC } \\
\text { VD }\end{array}$ \\
\hline
\end{tabular}

a This is the value estimated for $\lambda / 4$.

b Value of $\Delta H^{0}$ in $\mathrm{kJ} \cdot \mathrm{mol}^{-1}$.

c Value of $\Delta S^{0}$ in $\mathrm{J} \cdot \mathrm{deg}^{-1} \cdot \mathrm{mol}^{-1}$.

d Tris polypyridine complexes of osmium(III) and ruthenium(III).

e Estimated diffusion-controlled value. Estimated activation-controlled value is $8 \cdot 10^{9} \mathrm{M}^{-1} \cdot \mathrm{s}^{-1}$.

and +2 metal complexes is about 3.2 and $3.4 \cdot 10^{9}$ $\mathrm{M}^{-1} \cdot \mathrm{s}^{-1}$, respectively, at $0.1 \mathrm{M}$ ionic strength, using Eqn. 25 for the work term. One sees that the maximum experimental value (approx. $10^{8} \mathrm{M}^{-1}$. $\left.\mathbf{s}^{-1}\right)$ is substantially less than the diffusion-controlled value $\left(3 \cdot 10^{9} \mathrm{M}^{-1} \cdot \mathrm{s}^{-1}\right)$. Perhaps it represents an experimental result for the value of $S K_{\mathrm{A}} \kappa \nu$ for these Cyt $c$-metal complex systems (the estimated theoretical value above was approx. $8 \cdot 10^{9}$ $\mathbf{M}^{-1} \cdot \mathrm{s}^{-1}$ ). Some of the rate constants and other kinetic parameters that have been discussed in this section are summarized in Table VIII.

\section{VD. Photosynthetic reaction centers}

A $c$-type cytochrome is the electron donor to the oxidized bacteriochlorophyll dimer in the reaction centers of photosynthetic bacteria $[211,212]$ :

$\mathrm{c}^{2+}+(\mathrm{BChl})_{2}^{+} \rightarrow \mathrm{c}^{3+}+(\mathrm{BChl})_{2}$

We first consider kinetic data on reaction (Eqn. 48) for Chromatium vinosum and then consider the Rhodopseudomonas viridis reaction center complex, for which a crystallographic study has just become available, and for which there are kinetic data.

The rate of oxidation of ferrocytochrome $c$ by $(\mathrm{BChl})_{2}^{+}$in the reaction center of Chromatium vinosum is temperature-independent in the range $100-4 \mathrm{~K}\left(k=3 \cdot 10^{2} \mathrm{~s}^{-1}\right)$; at $300 \mathrm{~K}$ the electron transfer rate constant is about $10^{6} \mathrm{~s}^{-1}$, the pre-exponential factor appears to be $6 \cdot 10^{8} \mathrm{~s}^{-1}$, and the activation energy to be $17.6 \mathrm{~kJ} \cdot \mathrm{mol}^{-1}[19,212]$. $\Delta G^{0^{\prime}}$ for the reaction is $-43.5 \mathrm{~kJ} \cdot \mathrm{mol}^{-1}[19,213]$. From EPR measurements the center-to-center distance of Cyt $c$ and $(\mathrm{BChl})_{2}^{+}$is at least $2.5 \mathrm{~nm}$, corresponding to an edge-to-edge separation of $1.2-1.8 \mathrm{~nm}$ [214]. These two sets of results are examined next.

First we consider the high-temperature pre-exponential factor for the reaction in terms of the above model. This factor may be obtained from Eqn. 36b and hence equated (approximately) with $10^{13} \exp (-\beta d)$, neglecting any $\left|\Delta G^{0^{\prime}} / \lambda\right|$ compared with unity in Eqn. 35 and neglecting $\Delta S^{0^{\circ}} / R$ in Eqn. 36b; substitution of $\beta=12 \mathrm{~nm}^{-1}$ leads to $d=0.8 \mathrm{~nm}$, and so a nearest carbon to nearest carbon distance of $1.1 \mathrm{~nm}$ is calculated using the correction in subsection IVB. The value of $1.1 \mathrm{~nm}$ is close to the lower limit of the range of the separation distances (1.2-1.8 $\mathrm{nm}$ ) implicated by the EPR measurements [214] ", it would be useful to determine $\Delta S^{0}$ for reaction 48 , it having been neglected in the above calculation.

\footnotetext{
I In a quantum mechanical calculation an $H_{\mathrm{AB}}$ in Eqn. 12 was inferred from the experimental pre-exponential factor to be $1090 \mathrm{~kJ} \cdot \mathrm{mol}^{-1}[215,216]$. The calculation in Refs. 215,216 also explored a fitting of the detailed temperature dependence of the rates. In the model used $[215,216]$, both medium (solvent) and intramolecular modes were included.
} 
We turn next to the photosynthetic reaction center of Rhodopseudomonas viridis. The arrangement of the components has recently been determined from an X-ray crystal structure at 0.3 $\mathrm{nm}$ resolution [217]. In addition to protein, the crystallized reaction center complex contains four bacteriochlorophyll- $b$ and two bacteriopheophytin- $b$ molecules, four $c$-type cytochromes, one non-heme iron and one menaquinone. Two of the four BChl's are present as $(\mathrm{BChl})_{2}$ ('special pair') [218-220]), in which their pyrrole rings $I$ are stacked on top of each other about $0.3 \mathrm{~nm}$ apart. The distance between $\mathrm{BChl} \mathrm{Mg}$ atoms in the special pair is approx. $0.7 \mathrm{~nm}$ and the angle between the ring planes is about $15^{\circ}$. To each $\mathrm{BChl}$ of the special pair is associated another $\mathrm{BChl}$, whose $\mathrm{Mg}$ atom is $1.3 \mathrm{~nm}$ from the $\mathrm{Mg}$ atom of the special pair member, and the angle between the porphyrin planes of that member and the $\mathrm{BChl}$ is approx. $70^{\circ}$. Each of these monomeric $\mathrm{BChl}$ molecules is in turn also associated with a BPh molecule: the distance between the centers of their rings is 1.1 $\mathrm{nm}$, and the angle between the planes of their rings is approx. $64^{\circ}$. (This arrangement, in which there is a site between the special pair and the BPh had been predicted earlier so as to explain the effect ${ }^{\S}$ of a small magnetic field on the reaction kinetics for the closely related Rhodopseudomonas sphaeroides [221].) One of the BPh's is also in contact with a side chain of the menaquinone * The latter is, in turn, close to the non-heme iron.

A rectangular distribution of medium modes with a mean energy and width of $200 \mathrm{~cm}^{-1}$ was used, corresponding to $\lambda_{\mathrm{o}}=23.9 \mathrm{~kJ} \cdot \mathrm{mol}^{-1}$. The frequency used for the intramolecular mode was $500 \mathrm{~cm}^{-1}$ with $\lambda_{i}=222 \mathrm{~kJ} \cdot \mathrm{mol}^{-1}$. These parameters provided a good fit of the temperature dependence of the rate constants, except at the transition to temperature independence, which is more abrupt than calculated. However the $\lambda_{i}$ value seems unreasonably high - certainly much higher than $\lambda_{i}$ for the reactions of Cyt $c$ with typical metal complexes.

An alternative explanation has been given [129]; it presumes that the decay constant $\beta$ in Eqn. 37 is quite sensitive to orbital energies, a result inferred from calculations with delta function and square-well potentials. However, as discussed in subsection IIID, more recent calculations [71] show little difference between the optical and thermal matrix elements.

* A second quinone molecule expected for the other BPh molecule was not present and may have been lost during the preparation of the crystal [217].
The four BChl's, two BPh's, the menaquinone and the non-heme iron are believed to be situated within the photosynthetic membrane of the bacterial cell, with the non-heme iron near the inner membrane surface and the special pair near the outer surface. The four cytochrome $c$ molecules are located outside the membrane and are positioned roughly in a straight line in the crystal. The heme-iron of the cytochrome $c$ closest to the special pair is approx. $2.1 \mathrm{~nm}$ from each of the two magnesium centers of the $\mathrm{BChl}$ 's of the $(\mathrm{BChl})_{2}$. The four heme groups (of the cytochromes) can be viewed as forming two pairs, the pair closest to the $\mathrm{BChl}$ is related to the outer heme pair by rotation about an approximately 2-fold rotation axis. Within each pair the angle between the heme planes (i.e., the angle between the planes of the first and second hemes as well as the angle between the planes of the third and fourth hemes) is $78.5^{\circ} \pi$ and the iron-iron distance is approx. 1.4 $\mathrm{nm}$. The angle between the planes of the second and third hemes is $17.4^{\circ}$ and the distance between their iron centers is approx. $1.6 \mathrm{~nm}$.

Flash-induced redox titrations have shown that two different $c$-type cytochromes, designated cytochromes, $c-553$ and $c-558$ to indicate the positions of their $\alpha$-band maxima, are present in the Rps. viridis reaction center [222]. The midpoint potentials of the cytochromes are $-12 \mathrm{mV}$ and +330 $\mathrm{mV}$, respectively [222,223]. The midpoint potential of the BChl-b dimer is $+500 \mathrm{mV}$ at $\mathrm{pH} 8$ [223]. Both types of Cyt $c$ can reduce the (BChl $b)_{2}^{+}$at room temperature but only $c-553$ is capable of reducing it at $77 \mathrm{~K}[211,223]$. The immediate electron donor to the oxidized dimer at room temperature is the Cyt $c-558$ [223] *.

We first consider electron transfer within the linear arrangement of Cyt $c$ molecules. The separation of the iron centers of adjacent cytochromes

\footnotetext{
T We are grateful to Drs. J. Deisenhofer and H. Michel for this information.

* If the approximately linear arrangement of the cytochromes in the crystal of the reaction center also obtains in the bacterial cell, then the high-potential cytochrome (Cyt c-558) may be the only direct electron donor to the $(\mathrm{BChl})_{2}^{+}$; the apparent reduction of $(\mathrm{BChl})_{2}^{+}$by the low-potential cytochrome ( $c-553)$ then results from very rapid reduction of the Cyt $c-558$ by the Cyt $c-553$. This sequence has recently been entertained for Chromatium vinosum (see Ref. 20, p. 46).
} 
in the chain $(1.4-1.6 \mathrm{~nm})$ is similar to the separation of the iron centers found for the optimum docking of two Cyt $c$ molecules $(1.77 \mathrm{~nm}$, see subsection VB). Assuming that the $\lambda / 4$ and $\beta$ parameters for electron transfer in the Cyt $c$ chain are similar to those used for the Cyt $c$ exchange in solution ( $25 \mathrm{~kJ} \cdot \mathrm{mol}^{-1}$ and $12 \mathrm{~nm}^{-1}$, respectively) and using the correction in subsection IVB to obtain $d, k$ is calculated to be about $10^{6}-10^{7} \mathrm{~s}^{-1}$ for electron transfer between two adjacent $\mathrm{Cyt}$ $c-553$ or Cyt $c-558$ molecules and about $10^{7}-10^{8}$ $\mathrm{s}^{-1}$ for downhill electron transfer from Cyt $c-553$ to a neighboring Cyt $c-558$ molecule. These estimates do not allow for the effect of the low dielectric constant of the medium, which will be to lower $\lambda$ and increase $k$, and departure of the heme groups from co-planarity, which will tend to decrease $\kappa \nu$ and decrease $k$. These effects tend to cancel. It is interesting to note that the estimated rate constants for intrachain electron transfer are comparable to the rate constants for electron transfer from the terminal Cyt $c-558$ to the oxidized $\mathrm{BChl}-b$ dimer, a reaction discussed next.

The rate constant for reduction of the oxidized special pair by ferrocytochrome $c-558$ is $4 \cdot 10^{6}$ $\mathrm{s}^{-1}$ at room temperature [224]. The reduction of the special pair is presumably by the closest Cyt $c$ molecule. If the approx. $2.1 \mathrm{~nm}$ separation between the magnesium of the $(\mathrm{BChl})_{2}$ and the closest heme-iron in the crystal is also appropriate to the rate measurements, then the edge-to-edge separation of the chlorophyll and heme rings in the rate measurements is approx. $1.1 \mathrm{~nm}$ and, using the correction in subsection IVB, $d$ is approx. $0.8 \mathrm{~nm}$. The driving force $\left(-\Delta G^{0^{\prime}}\right)$ associated with the rate constant of $4 \cdot 10^{6} \mathrm{~s}^{-1}$ is 170 $\mathrm{mV}$ or $16.3 \mathrm{~kJ} \cdot \mathrm{mol}^{-1}$ and the reaction is expected to have only a low $\Delta G_{r}^{*}$ in Eqn. 31. The value of $\lambda$ for the reaction is presently not known though a rough estimate of about $12 \mathrm{~kJ} \cdot \mathrm{mol}^{-1}$ (based on a barrierless result) is made for $(\mathrm{BChl})_{2}, \mathrm{BChl}$ and $\mathrm{BPh}$ in Appendix II. Using the value for $\lambda / 4$ of 25 $\mathrm{kJ} \cdot \mathrm{mol}^{-1}$ for the Cyt $c-\mathrm{Cyt} c$ exchange reaction given earlier the estimated $\lambda$ for the $\left(\mathrm{BChl}_{2}^{+}-\mathrm{Cyt}\right.$ $c$ reaction is the averaged of these, namely about $56.5 \mathrm{~kJ} \cdot \mathrm{mol}^{-1}$. Using the above driving force, the $\Delta G_{\mathrm{r}}^{*}$ in Eqn. 31 is estimated to be $7.1 \mathrm{~kJ} \cdot \mathrm{mol}^{-1}$. From Eqns. 31-33a and 37b the predicted rate constant is $1 \cdot 10^{13} \mathrm{e}^{-12 \cdot 0.8} \mathrm{e}^{-1.6 / R T}$ or $4 \cdot 10^{7} \mathrm{~s}^{-1}$, using $\beta=12 \mathrm{~nm}^{-1}$ and $\mathrm{d}=0.8 \mathrm{~nm}$. This value may be compared with the experimental value [224] of $4 \cdot 10^{6} \mathrm{~s}^{-1}$ found for reaction 48 in Rps. viridis.

Picosecond flash-photolysis techniques have been used to study the primary electron-transfer reactions in reaction centers and in a variety of model photosynthetic systems [226-229]. An important result of the studies with model systems is that the forward electron transfer from an excited porphyrin monomer or dimer to an electron acceptor (usually a quinone) can be very rapid while the back reaction to reform ground-state reactants, which is even more exothermic, can be relatively slow, depending on the system and solvent used. The forward reactions generally lie in the normal free-energy region, and it has been proposed that the back reactions are slow because they lie in the inverted region.

In reaction centers of Rps. sphaeroides electron donation from the excited singlet state of $(\mathrm{BChl})_{2}$ to BPh occurs in 4 ps (Kirmaier, C., Holten, D. and Parson, W.W., personal communication) * and the driving force for the reaction has been reported to be $0.13 \mathrm{eV}$ though perhaps it may be somewhat larger ". Some spectroscopic evidence (a 'shoulder') has been offered that the reaction involves the formation of $\mathrm{BChl}^{-}$as a distinct

The resulting activation energy is $7.1 \mathrm{~kJ} \cdot \mathrm{mol}^{-1}$, if $\Delta S^{0}$ can be neglected. Its experimental value has not yet been determined apparently, but the experimental value for $R p s$. gelatinosa is $9.6 \mathrm{~kJ} \cdot \mathrm{mol}^{-1}$ [225].

* Current work (Kirmaier, C., Holten, D. and Parson, W.W., personal communication) on this rate constant is consistent with the earlier value [230]. Very recently, evidence for possible formation of $\mathrm{BChl}^{-}$in $1 \mathrm{ps}$ and $\mathrm{BPh}^{-}$in $5 \mathrm{ps}$ has been reported [231].

T The driving force is based on estimated potentials $[228,234,235]$ for reduction of $(\mathrm{BChl})_{2}^{+}$to excited $(\mathrm{BChl})_{2}$ and of $\mathrm{BPh}$ to $\mathrm{BPh}^{-}$. Recently, delayed fluorescence measurements gave a driving force of $0.16 \mathrm{eV}$ [236]. In Rps. viridis the estimated potentials $[223,228,237]$ lead to a driving force of $0.12 \mathrm{eV}$. However, the in vivo measurements for $\mathrm{BPh} / \mathrm{BPh}^{-}$in each case appear to be made with the $\mathrm{Q}$ present. If it were present as $\mathrm{Q}^{-}$rather than as $\mathrm{QH}$, the $\mathrm{Q}^{-}$ would destabilize the $\mathrm{BPh}^{-}$and so make the calculated driving force too small. The dielectric constant $D_{\mathrm{s}}$ of the membrane has been reported to be about 3.8 [238] and has been estimated to be $30 \%$ less (Dutton, P.L., personal communication). Two negative charges a distance $1.5 \mathrm{~nm}$ apart in a medium of $D_{s}=3$ have a repulsive interaction of $0.33 \mathrm{eV}$. 
intermediate [232]. (However, such evidence does not appear to be definitive.) From the crystal structure the closest approach (carbon-to-carbon) of the porphyrin rings in $(\mathrm{BChl})_{2}$ and $\mathrm{BChl}$ appears to be about $0.35-0.4 \mathrm{~nm}$, and about $0.4 \mathrm{~nm}$ for the $\mathrm{BChl}$ to $\mathrm{BPh}$ rings (from the $\mathrm{Mg}$ in the $\mathrm{BChl}$ to the acetyl group in the BPh). Since the reaction is barrierless (negligible activation energy [232]) we shall take $-\Delta G^{0^{\prime}} \cong \lambda$ (Appendix II) ${ }^{\S}$. Then, using $\beta=12 \mathrm{~nm}^{-1}$ and $d=0.1 \mathrm{~nm}$ (after subtracting $0.3 \mathrm{~nm}$ from the $0.4 \mathrm{~nm}$ distance as in subsection IVB) a half-life of the order of $0.2 \mathrm{ps}$ is calculated, as in Appendix II.

The half-life for the electron transfer from $\mathrm{BPh}^{-}$ to quinone in Rps. sphaeroides is $200 \mathrm{ps}$ at room temperature and $100 \mathrm{ps}$, independent of temperature, below $100 \mathrm{~K}$ [231]. Thus, there is a negligible activation energy. The driving force for the reaction at room temperature is, incidentally, approx. $0.61 \mathrm{eV}[228,241]$. The corresponding values in Rps viridis are $4 \cdot 10^{9} \mathrm{~s}^{-1}$ and approx. $0.47 \mathrm{eV}$ at room temperature $[224,223]$. When the calculated $k$ is written as $1 \cdot 10^{13} \exp (-\beta d) \mathrm{s}^{-1}$, with $\beta \approx 12$ $\mathrm{nm}^{-1}$ and $d$ estimated from the crystal structure to be in the vicinity of $1.0 \mathrm{~nm}$ minus the $0.3 \mathrm{~nm}$ correction (subsection IVB), the calculated $k$ is about $5 \cdot 10^{9} \mathrm{~s}^{-1}$, corresponding to a half-life of about 140 ps. The calculated value at $0 \mathrm{~K}$ (Appendix II) is only slightly greater than this.

In other experiments on the rate constant for the $\mathrm{BPh}^{-}+\mathrm{Q}$ reaction, the nature of $\mathrm{Q}$ has been varied, causing the $-\Delta G^{0}$ of the reaction to change by about $0.2 \mathrm{eV}$ [242]. The resulting variations in the rate constant were only a factor of two or so [242] and, perhaps due to other changes caused by

The potentials for the reduction of $(\mathrm{BChl})_{2}^{+}$to excited $(\mathrm{BChl})_{2}$ were calculated from the energy for the ground state to singlet transition $E_{0-0}(0 \rightarrow 0$ levels $)$ and the reduction potential for the ground state $(\mathrm{BChl})_{2}^{+} /(\mathrm{BChl})_{2}$ couple. $E_{0-0}$ equals the energy of the absorption maximum $h \nu_{\max }$ minus one-half the Stokes shift [240]. Values for the Stokes shift $\left(450 \mathrm{~cm}^{-1}\right)$ for Rps. sphaeroides are given in Ref. [234].

$\$$ The self-exchange rate constants $k$ for the $\mathrm{Chl}^{+/ 0}$ and $\mathrm{BPh}^{0 /-}$ pairs are about $2-4 \cdot 10^{6} \mathrm{M}^{-1} \cdot \mathrm{s}^{-1}$ in a somewhat polar solvent (methanol/methylene chloride) [233]. If $k$ is written as $1 \cdot 10^{11} \exp ^{-\lambda / 4 R T} \mathrm{M}^{-1} \cdot \mathrm{s}^{-1}$, then $\lambda / 4$ is estimated to be about $14.6 \mathrm{~kJ} \cdot \mathrm{mol}^{-1}$. In the less polar membrane system, $\lambda / 4$ would be less. The value of $\lambda$ is estimated in Appendix II to be about $25 \mathrm{~kJ} \cdot \mathrm{mol}^{-1}$ or less. the substitution of $\mathrm{Q}$, were somewhat random. From Eqns. 1-3 it can be seen that in the vicinity of the maximum rate, i.e., when $-\Delta G^{0} \approx \lambda$, a change $\delta \Delta G^{0}$, causes the rate constant to change by a factor of $\exp \left[-\left(\delta \Delta G^{0^{\prime}}\right)^{2} / 4 \lambda R T\right]$. With a value of $\lambda$ of about $0.5 \mathrm{eV}$, the reaction is barrierless at $-\Delta G^{0^{\prime}} \approx 0.5 \mathrm{eV}$ and the above rate changes only by a factor of two when $\left|\delta \Delta G^{0^{\prime}}\right|=0.2 \mathrm{eV}$, a result consistent with the data. Again, when a second quinone $Q_{B}$ was present as $Q_{B}^{-}$, the rate constant for the $\mathrm{BPh}^{-}+\mathrm{Q}_{\mathrm{A}}$ electron transfer was smaller by almost a factor of two [243]. The presence of $\mathrm{Q}_{\mathrm{B}}^{-}$is calculated to reduce the driving force $-\Delta G^{0^{\prime}}$ by perhaps several tenths of a volt, due to the coulombic repulsion of the $\mathrm{Q}_{\mathrm{A}}^{-}$and $\mathrm{Q}_{\mathrm{B}}^{-}$ in the low dielectric constant medium ". Such an effect is calculated, as above, to reduce $k$ by a factor of near 2 from its value at $-\Delta G^{0^{\prime}} \approx \lambda$.

In a model porphyrin-quinone study [38] the maximum rate constant for the reduction of the quinone by the excited porphyrin (occurring at $-\Delta G^{0^{\prime}} \approx \lambda$ ) was reported as $2 \cdot 10^{11} \mathrm{~s}^{-1}$. The carbon-carbon distance at closest approach of the reacting partners was $0.6-0.7 \mathrm{~nm}$ [244], giving a $d$ of about $0.4 \mathrm{~nm}$ after using the $0.3 \mathrm{~nm}$ correction in subsection IVB. The calculated maximum rate constant, $1 \cdot 10^{13} \exp (-\beta d)$, is about $8 \cdot 10^{10} \mathrm{~s}^{-1}$, which is close to the reported value of $2 \cdot 10^{11} \mathrm{~s}^{-1}$.

The various results in this section are summarized in Table IX.

$V E$. Reactions of cytochrome $c$ with cytochrome $b_{5}$ and cytochrome $c$ oxidase

In this section we consider the electron-transfer reactions of cytochrome $c$ with cytochrome $b_{5}$ and with cytochrome $c$ oxidase. The first reaction involves electron transfer between cytochrome $c$ and a heme protein of opposite charge and the second involves electron transfer of cytochrome $c$ with its natural partner.

It will be recalled that cytochrome $b_{5}$ differs from cytochrome $c$ in several respects. The fifth and sixth coordination sites of the heme in cy-

\footnotetext{
As pointed out in an earlier footnote, two negative charges a distance $1.5 \mathrm{~nm}$ apart in a medium of estimated dielectric constant of about 3 has a repulsive interaction energy of about $0.33 \mathrm{eV}$.
} 
TABLE IX

ABSOLUTE RATES FOR ELECTRON-TRANSFER REACTIONS IN PHOTOSYNTHETIC REACTION CENTERS

\begin{tabular}{llll}
\hline Reaction $^{\mathrm{a}}$ & $\begin{array}{l}\Delta G^{0 \mathrm{~b}} \\
\left(\mathrm{~kJ} \cdot \mathrm{mol}^{-1}\right)\end{array}$ & $\tau_{\text {obsd }}$ & $\tau_{\text {calcd }}$ \\
\hline $\mathrm{Cyt}^{\mathrm{II}} \sim \mathrm{BChl}_{2}^{+}$ & -16.3 & $0.2 \mu \mathrm{s}$ & $0.02 \mu \mathrm{s}$ \\
${ }^{*}\left(\mathrm{BChl}_{2} \sim \sim \mathrm{BChl}_{2} \sim \sim \mathrm{BPh}\right.$ & $-21(?)$ & $\approx 1-4 \mathrm{ps}^{\mathrm{c}}$ & $>0.2 \mathrm{ps}$ \\
$\mathrm{BPh}^{-} \sim \sim \mathrm{Q}$ & -50 & $100-200 \mathrm{ps}$ & $140 \mathrm{ps}$ \\
$\mathrm{BPh}^{-} \sim \sim \mathrm{Q}_{\mathrm{A}}, \mathrm{Q}_{\mathrm{B}}^{-}$present & $\approx 0.2 \mathrm{eV}$ & increases & increases \\
& & factor of & factor of 2 \\
$\mathrm{BPh}^{-} \sim \sim \mathrm{Q}, \mathrm{Q}$ varied & $\approx 0.2 \mathrm{eV} \mathrm{s}$ & changes & factor of 2 \\
${ }^{*}$ Porphyrin $\sim \mathrm{Q}$ & $-\lambda$ & factor of 2 & $\tau_{\min } \approx 9 \mathrm{ps}$ \\
\hline
\end{tabular}

asterisks indicate electronically excited states.

b The $\Delta G^{0}$ values are approximate.

c Assignment of time constants is uncertain.

d The value of $\tau_{\text {calcd }}$ is larger than this value if smaller $\lambda$ 's are used, i.e., $\tau_{\text {calcd }}$ is about 1 ps when $\lambda=5 \mathrm{~kJ} \cdot \mathrm{mol}^{-1}$ (see also Appendix II).

e Value depends on temperature.

f This is the approximate decrease in $\left|\Delta G^{0}\right|$ due to the presence of $\mathrm{Q}_{\mathrm{B}}^{-}$.

g This is the approximate variation in $\left|\Delta G^{0}\right|$ as $Q$ is varied.

tochrome $b_{5}$ are occupied by $\mathrm{N}$ atoms of histidine imidazole groups and the porphyrin ring is not covalently bonded to the protein (other than through the coordinated histidines). In addition, the net charge of ferricytochrome $b_{5}$, calculated from its amino acid composition, is -9 [245] and there are four negatively-charged carboxyl groups in the heme crevice region. By contrast, the net charge of ferricytochrome $c$ is +8 and the heme is surrounded by four positively charged lysine residues. Therefore, unlike the corresponding exchange reactions, interaction (of the hemes) of cytochrome $c$ and cytochrome $b_{5}$ is attractive. By using computer modeling, Salemme [246,247] has shown that ferricytochrome $c$ and ferrocytochrome $b_{5}$ can form a complex in which the heme groups of the two proteins are nearly coplanar with their edges separated by $0.84 \mathrm{~nm}$ at first contact of the surface amino-acid side chains. Additional support for the formation of this complex has been obtained from protein modification studies $[248,249]$ (although the more recent results implicate interactions between five complementary pairs, rather than just four as originally proposed) and from energy transfer measurements using zinc-substituted cytochrome $c$ [154].

The second-order rate constant for the oxidation of ferrocytochrome $b_{5}$ by ferricytochrome $c$ is
$3 \cdot 10^{8}$ and $3 \cdot 10^{7} \mathrm{M}^{-1} \cdot \mathrm{s}^{-1}$ at 0.02 and $0.10 \mathrm{M}$ phosphate $(\mu=0.04$ and $0.20 \mathrm{M})(\mathrm{pH} \mathrm{7})$, respectively [249]. (The latter value is very similar to that reported earlier [250].) The association involves two oppositely-charged reactants, and so the equilibrium constant for this association is, as expected, very dependent on ionic strength [251]. The experimental binding constant (expressed in concentrations rather than activities) for the reactants is approx. $10^{5} \mathrm{M}^{-1}$ at $\mu=0.01 \mathrm{M}$ and approx. $5 \cdot 10^{7} \mathrm{M}^{-1}$ at $\mu=0$ [251]. The extrapolation to higher ionic strengths is highly uncertain, and we tentatively use a value of approx. $10^{3} \mathrm{M}^{-1}$ for the binding constant at $\mu=0.04 \mathrm{M}$ ?.

Like cytochrome $c$, the structures of oxidized and reduced cytochrome $b_{5}$ are essentially identical [252]. In view of the similarity of the electron transfer distance $d$ and the structures of the heme sites, the value of $S \kappa \nu$ is unlikely to be very

\footnotetext{
T A binding constant calculated from $S=0.01$ and a $K_{\mathrm{A}}$ obtained from Eqns. 25 and 57 is only about $2-3 \mathrm{M}^{-1}$ for these oppositely charged reactants. The contrast between this value and the extrapolated experimental binding constant suggests that for oppositely charged reactants in contact the local electrostatic charges may dominate the interaction and require that the delocalized-charge expression Eqn. 25 be replaced by a more complex one such as that in Refs. 174-177.
} 
different for the cytochrome $c$ exchange reactions and the ferricytochrome $c$-ferrocytochrome $b_{5}$ cross-reaction. Thus, the $10^{5}$-fold difference in the rate constants (at $\mu=0.1 \mathrm{M}$ ) for these two reactions may reflect several factors, including a difference in driving force $\left(-\Delta G^{0}=0.26 \mathrm{eV}\right.$ $[160,253])$, which contributes a factor $\exp \left(-\Delta G^{0} / 2 R T\right)$ or 150 , and a difference in the stability constants of the precursor complexes (a factor of $10^{3}$ if the experimental $K_{\mathrm{A}}$ for $\mathrm{Cyt}$ $c$-Cyt $b_{5}$ is used).

The ferricytochrome $c$-ferrocytochrome $b_{5}$ bimolecular rate constant and binding constant may be used (Appendix IB) to obtain a first-order rate constant for a complexed pair, namely $3 \cdot 10^{8} / 10^{3}$ or $3 \cdot 10^{5} \mathrm{~s}^{-1}$ at $\mu=0.04 \mathrm{M}$ and $\mathrm{pH} 7$, or if an $S=0.01$ is also to be introduced, $3 \cdot 10^{7} \mathrm{~s}^{-1}$. This value may be compared with that calculated from Eqns. 31-33 with $\left|\Delta G^{0^{\prime}} / \lambda\right| \ll 1$ : the rate constant calculated using (as above) $\lambda / 4 \approx 25 \mathrm{~kJ} \cdot \mathrm{mol}^{-1}$, $\beta=12 \mathrm{~nm}^{-1}, d \approx 0.54 \mathrm{~nm}$ and $-\Delta G^{0}=0.30 \mathrm{eV}$ at $\mu=0.04 \mathrm{M}(0.02 \mathrm{M}$ phosphate buffer $)[160,253]$ equals $10^{13} \mathrm{e}^{-12 \cdot 0.54} \mathrm{e}^{-10} \mathrm{e}^{5.8}$ or $2 \cdot 10^{8} \mathrm{~s}^{-1}$, which should be compared with the value of $3 \cdot 10^{5} \mathrm{~s}^{-1}$ or $3 \cdot 10^{7} \mathrm{~s}^{-1}$ obtained above. There are, however, some puzzling features ${ }^{\S}$.

Next we consider the reaction of cytochrome $c$ with its physiological partner, cytochrome $c$ oxidase. The functional unit of mammalian cytochrome oxidase, it will be recalled, contains two heme-A residues, namely cytochromes $a$ and $a_{3}$, differing only in their axial ligands, and two copper

\footnotetext{
$\$$ Pulse radiolysis experiments on the Cyt $c-$ Cyt $b_{5}$ complex itself show a low $\left(1.4 \cdot 10^{3} \mathrm{~s}^{-1}\right)$ value for $k$ (McLendon, G.L. and Miller, J.R., unpublished results). Also puzzling is the value of $5 \cdot 10^{3} \mathrm{~s}^{-1}$ reported [154] for the $k$ for quenching (presumably by electron transfer) of a triplet in a zinc-substituted Cyt $c$ with ferricyt $b_{5}$. The driving force of this reaction is about $1 \mathrm{eV}$ [255], $d$ is believed to be small (approx. $0.5-0.6 \mathrm{~nm}$ ) and yet $k$ is relatively small. In a plot of $\ln k$ vs $\Delta G^{0}$, for related systems the maximum $k$ was $5 \cdot 10^{5} \mathrm{~s}^{-1}$ which is also relatively low for the small value of $d$ inferred above from computer modeling studies. Possible interpretations include a prior conformational change or a wrong docking of the two proteins. In this connection it is of interest to note that the oxidation of ferrocytochrome $b_{5}$ by metal complexes and the competitive inhibition of the reaction by redox-inactive complexes are consistent with electron transfer through the exposed heme edge and an effective charge of -4 at the oxidant binding site [245].
}

atoms [256], one of which, termed 'copper A', is magnetically isolated (although the existence of a weak magnetic dipolar interaction between the $\mathrm{Cu}_{\mathrm{A}}$ and cytochrome $a$ has been recently proposed [257]). The $\mathrm{Cu}_{\mathrm{A}}$ is detectable by EPR in the cupric state. The first step in the anaerobic oxidation of ferrocytochrome $c$ by cytochrome oxidase is the very rapid oxidation of ferrocytochrome $c$ by cytochrome $a$ with a rate constant of (8-9) $\cdot 10^{6}$ $\mathrm{M}^{-1} \cdot \mathrm{s}^{-1}$ at $\mathrm{pH} 7.4$ and $25^{\circ} \mathrm{C}$; the estimates of the equilibrium constant for this reaction range from 1 to $10^{2}[258-260]$. For a binding constant of approx. $10^{5} \mathrm{M}^{-1}$ [261], the equivalent first-order rate constant for this step is (8-9) $10^{6} / 10^{5} \mathrm{~s}^{-1}$; i.e., approx. $10^{2} \mathrm{~s}^{-1}$ (Appendix IB). The reaction of ferrocytochrome $c$ with cytochrome $a$ is followed by a slower step $\left(k \approx 40-100 \mathrm{~s}^{-1}\right)$, presumably intramolecular electron transfer from the reduced cytochrome $a$ to copper A, for which a separation distance of 1.3-2.6 $\mathrm{nm}$ has been estimated [257]:

$$
\begin{aligned}
& \mathrm{c}^{2+}+[\mathrm{a}]^{3+}\left[\mathrm{Cu}_{\mathrm{A}}\right]^{2+} \rightleftharpoons \mathrm{c}^{3+}+[\mathrm{a}]^{2+}\left[\mathrm{Cu}_{\mathrm{A}}\right]^{2+} \\
& {[\mathrm{a}]^{2+}\left[\mathrm{Cu}_{\mathrm{A}}\right]^{2+} \rightarrow[\mathrm{a}]^{3+}\left[\mathrm{Cu}_{\mathrm{A}}\right]^{+} .}
\end{aligned}
$$

The very rapid oxidation of ferrocytochrome $c$ by cytochrome $a$ is noteworthy in the light of the small driving force for the reaction $(<12 \mathrm{~kJ}$. $\left.\mathrm{mol}^{-1}[258-260]\right)$ and the large distance between the heme groups, which has been estimated to be approx. $2.5 \mathrm{~nm}$ [262]. If the latter distance permits a heme-to-heme edge separation of $1.2-1.5 \mathrm{~nm}$, then the above rate constants can be rationalized using the typical parameters derived earlier. For example, for modest driving forces (approx. 10.5 $\mathrm{kJ} \cdot \mathrm{mol}^{-1}$ ) and small $\lambda / 4$ parameters (approx. 25 $\mathrm{kJ} \cdot \mathrm{mol}^{-1}$ ), a rate constant of the order of $50 \mathrm{~s}^{-1}$ is consistent with an electron transfer distance $d$ of approx. $1.5 \mathrm{~nm}$ if $\beta \approx 12 \mathrm{~nm}^{-1}$.

\section{$V F$. Reactions of blue copper proteins}

We consider next the reaction of cytochrome $c$ with copper proteins and also other protein-protein reactions. It will be recalled that many copper-containing proteins contain a 'type 1' or 'blue' copper center which imparts an intense blue color to these substances in their copper(II) forms. Such copper centers are present in the electron 
carriers plastocyanin, azurin and stellacyanin, which each contain one copper atom per molecule [263]. X-ray structural work has shown that the copper sites in poplar plastocyanin and in Pseudomonas aeruginosa azurin are very similar $[264,265]$. In each protein the blue copper is bound to two nitrogen and two sulfur atoms: the copper atom is coordinated by two histidine imidazolate groups, a cysteine thiolate, and a methionine thioether group. Although its structure has not been determined, stellacyanin must have at least one copper ligand different from that in plastocyanin and azurin, since the protein does not contain methionine. It has been suggested that in stellacyanin the site normally occupied by methionine is filled by a disulfide group [266].

After cytochrome $c$, plastocyanin is probably the best characterized metalloprotein, at least so far as electron-transfer properties are concerned. NMR studies have implicated two sites for the reactions of plastocyanin with metal complexes [267]. The first site is an uncharged region situated near the copper. This site may provide an electron transfer pathway via the exposed imidazole ring of His- 87 and is believed to be the site used by negatively charged oxidants such as $\mathrm{Fe}(\mathrm{CN})_{6}^{3-}$ [166]. The second site is a negatively charged region near Tyr-83 which is about $1.2 \mathrm{~nm}$ from the copper [267]. Electron transfer to and from this region may occur via the $\pi$-systems of aromatic amino acids and may be the site used by positively-charged oxidants such as Co(phen $)_{3}^{3+}$ [164,197,268-272].

Reactions of the blue copper proteins with a variety of metal complexes have been studied [164,190,197,268-274]. Some representative examples together with the rate constants calculated from Eqn. 42 are presented in Table X. The measured self-exchange rate constants for the proteins are: azurin, $2 \cdot 10^{6} \mathrm{M}^{-1} \cdot \mathrm{s}^{-1}$ at $50^{\circ} \mathrm{C}$ [276], plastocyanin, up to $2 \cdot 10^{4} \mathrm{M}^{-1} \cdot \mathrm{s}^{-1}$ at $50^{\circ} \mathrm{C}$ [277]; stellacyanin, $1.2 \cdot 10^{5} \mathrm{M}^{-1} \cdot \mathrm{s}^{-1}$ at $20^{\circ} \mathrm{C}$ [278]. In order to calculate the $k_{12}$ values, the azurin and plastocyanin self-exchange rates were corrected to $25^{\circ} \mathrm{C}$ on the assumption that the activation energies for these exchanges are comparable to that for cytochrome $c$. The self-exchange rates for azurin and plastocyanin at $25^{\circ} \mathrm{C}$ are then calculated to be $10^{5} \mathrm{M}^{-1} \cdot \mathrm{s}^{-1}$ and up to $10^{3} \mathrm{M}^{-1} \cdot \mathrm{s}^{-1}$, respectively. We have used the upper limit for the plastocyanin exchange rate constant in calculating the $k_{12}$ values in Table $\mathrm{X}$. Comparison of the observed and calculated $k_{12}$ values shows that plastocyanin and stellacyanin are better behaved than azurin, at least as regards their cross-reactions $[272,273,279]$ "

In the case of azurin, the calculated values of the rates of the cross-reactions with cationic metal complexes are at least comparable with those predicted from the cross-relation. However, anomalously low values for the rates are obtained when the reaction is with anionic metal complexes (Table $\mathrm{X}$ ). This anomaly is also present, but to a lesser extent, for plastocyanin and in a milder way for cytochrome $c$ (Table VII). Although only a limited amount of data is available, it does appear that these reactions with anionic metal complexes are anomalous. Perhaps there is specific binding of the anions. Anions tend, for example, to be more readily bound at interfaces than do cations of comparable size, a known result in electrochemistry, and there is expected to be an interplay between binding and electrostatics. There may even be multiple binding sites. Precursor complexes of ferrocyanide with azurin [280] and complexities for the cytochrome $c-\mathrm{Fe}(\mathrm{CN})_{6}^{3-/ 4-}$ reaction [195,196] have been described. Different electron-transfer distances may also be involved [281].

It has proved possible to exploit binding at the Tyr-83 site of plastocyanin to obtain information about the distance dependence of electron transfer from this site to the copper center. In a recent study $\mathrm{Cr}$ (phen) ${ }_{3}^{3+}$ bound near the Tyr- 83 of reduced plastocyanin was excited using a laser flash-photolysis technique. The $\mathrm{Cr}(\mathrm{phen})_{3}^{3+}$ excited state produced is a very powerful electron acceptor and was found to oxidize the reduced plastocyanin with a concentration-independent rate constant of $3 \cdot 10^{6} \mathrm{~s}^{-1}$. If it is assumed that the electron transfer is barrierless because of the high driving force of the reaction, then a value of

\footnotetext{
As noted earlier in connection with the cytochrome $c$ studies, an incorrect self-exchange rate for the $\mathrm{Ru}\left(\mathrm{NH}_{3}\right)_{5} \mathrm{py}^{3+/ 2+}$ couple has been used in certain other cross-relation applications. This has resulted in some erroneous conclusions regarding the validity of the cross-relation.
} 
TABLE X

COMPARISON OF OBSERVED AND CALCULATED RATE CONSTANTS FOR THE REACTIONS OF BLUE COPPER PROTEINS WITH METAL COMPLEXES

Reactions at $25^{\circ} \mathrm{C}, \mathrm{pH} 7$ and $0.5 \mathrm{M}$ ionic strength unless otherwise noted. The rate constants were calculated using Eqn. 42 and the following exchange rates and redox potentials [272] for the copper proteins: azurin, $1 \cdot 10^{5} \mathrm{M}^{-1} \cdot \mathrm{s}^{-1}, 0.31 \mathrm{~V}$; plastocyanin, $1 \cdot 10^{3}$ $\mathrm{M}^{-1} \cdot \mathrm{s}^{-1}, 0.36 \mathrm{~V}$; stellacyanin, $1.2 \cdot 10^{5} \mathrm{M}^{-1} \cdot \mathrm{s}^{-1}, 0.19 \mathrm{~V}$. Net charges on the proteins (oxidized/reduced) are: azurin $(-1 /-2)$, plastocyanin $(-9 /-10)$, stellacyanin $(0 / 0)[194,273]$. The zero net charge on stellacyanin is based on the ionic-strength independence of its reaction with $\mathrm{Co}(\mathrm{phen})_{3}^{3+}[164]$. For additional data, see Table VII.

\begin{tabular}{llllll}
\hline Protein & Reagent & $K_{12}$ & $\begin{array}{l}k_{12, \text { obsd }} \\
\left(\mathrm{M}^{-1} \cdot \mathrm{s}^{-1}\right)\end{array}$ & $\begin{array}{l}k_{11, \text { calcd }} \\
\left(\mathrm{M}^{-1} \cdot \mathrm{s}^{-1}\right)\end{array}$ & Ref. \\
\hline $\begin{array}{lllll}\text { Azurin } \\
\text { (R. aeruginosa })\end{array}$ & $\mathrm{Co}(\mathrm{phen})_{3}^{3+}$ & 7.0 & $4.4 \cdot 10^{3}$ & $5.6 \cdot 10^{3}$ & 194 \\
& $\mathrm{Ru}\left(\mathrm{NH}_{3}\right)_{5} \mathrm{py}^{3+}$ & 1.5 & $2.0 \cdot 10^{3 \mathrm{a}}$ & $1.3 \cdot 10^{5}$ & 190 \\
& $\mathrm{Fe}(\mathrm{EDTA})^{2-}$ & $1.6 \cdot 10^{3}$ & $1.3 \cdot 10^{3}$ & $2.2 \cdot 10^{6}$ & 270 \\
Plastocyanin & $\mathrm{Co}(\mathrm{ox})_{3}^{3-}$ & $2.5 \cdot 10^{4}$ & $2.9 \cdot 10^{-2}$ & $2.3 \cdot 10^{1}$ & 194 \\
(spinach) & $\mathrm{Co}(\mathrm{phen})_{3}^{3+}$ & 1.0 & $1.0 \cdot 10^{3}$ & $2.1 \cdot 10^{2}$ & 194 \\
& ${\mathrm{Ru}\left(\mathrm{NH}_{3}\right)_{5} \mathrm{py}^{3+}}^{\mathrm{Fe}(\mathrm{EDTA})^{2-}}$ & 0.21 & $7.1 \cdot 10^{3 \mathrm{a}}$ & $4.8 \cdot 10^{3}$ & 190 \\
& $\mathrm{Co}(\mathrm{ox})_{3}^{3-}$ & $1.1 \cdot 10^{4}$ & $8.2 \cdot 10$ & $5.8 \cdot 10^{5}$ & 270 \\
Stellacyanin & $3.5 \cdot 10^{3}$ & $2.4 \cdot 10^{-1}$ & $9.1 \cdot 10^{-1}$ & 194 \\
$($ R. vernicifera $)$ & $\mathrm{Co}(\mathrm{phen})_{3}^{3+}$ & $7.5 \cdot 10^{2}$ & $1.8 \cdot 10^{5}$ & $6.3 \cdot 10^{4}$ & 194 \\
& $\mathrm{Ru}\left(\mathrm{NH}_{3}\right)_{5} \mathrm{py}^{3+}$ & $1.6 \cdot 10^{2}$ & $1.9 \cdot 10^{5 \mathrm{a}}$ & $1.5 \cdot 10^{6}$ & 190 \\
& $\mathrm{Fe}(\mathrm{EDTA})^{2-}$ & $1.5 \cdot 10^{1}$ & $4.3 \cdot 10^{5}$ & $2.3 \cdot 10^{5}$ & 270 \\
& $\mathrm{Co}(\mathrm{ox})_{3}^{3-}$ & $2.6 \cdot 10^{6}$ & $7.3 \cdot 10^{2}$ & $2.7 \cdot 10^{2}$ & 194 \\
\hline
\end{tabular}

a Ionic strength, $0.1 \mathrm{M}$.

b Ionic strength, $0.2 \mathrm{M}$.

$\beta \approx 12 \mathrm{~nm}^{-1}$ and an intramolecular electron-transfer distance $d$ of $1.0 \mathrm{~nm}$ yields a rate of $6 \cdot 10^{6} \mathrm{~s}^{-1}$ [282].

Bimolecular reaction rate constants of plastocyanin $\left(\mathrm{Cu}^{\mathrm{l}}\right.$ and $\left.\mathrm{Cu}^{\mathrm{II}}\right)$ with various metal complexes have been measured for reactions having very negative $\Delta G^{0}$ 's, and it is interesting to compare the results with those of cytochrome $c$. The reaction of plastocyanin $\mathrm{Cu}^{\mathrm{I}}$ with the excited state of $\mathrm{Ru}(\mathrm{bpy})_{3}^{2+}$, of $\mathrm{Cu}^{\mathrm{II}}$ with $\mathrm{Cr}$ (phen $)_{3}^{2+}$, of $\mathrm{Cu}^{\mathrm{I}}$ with the excited state of $\mathrm{Cr}(\mathrm{phen})_{3}^{3+}$ and of $\mathrm{Cu}^{\mathrm{II}}$ with excited $\mathrm{Ru}(\mathrm{bpy})_{3}^{2+}$, for which $-\Delta G^{0^{\prime}}$ is 0.48 , $0.64,1.06$ and $1.20 \mathrm{~V}$, respectively, have rate constants of $1.9,2.3,3.5$ and 4.2 , in units of $10^{9}$ $\mathbf{M}^{-1} \cdot \mathrm{s}^{-1}$, respectively [281]. The diffusion-controlled rate constants $k_{\mathrm{D}}$ calculated (for spherically symmetric reactants) using Eqns. 25 and 53b are all about $6 \cdot 10^{9} \mathrm{M}^{-1} \cdot \mathrm{s}^{-1}$. The above values are perhaps somewhat less than this, but the difference is small, and one possible explanation is mentioned briefly in Appendix IB. Another possibility is that the calculation of a diffusion-con- trolled rate constant corrected for electrostatic effects is approximate.

We next consider the reactions of the blue copper proteins with other proteins as well as some other protein-protein reactions. Observed and calculated rate constants for these systems are compared in Table XI. Except for the reaction of plastocyanin with cytochrome $c$, in which the reactants are of very different charge types, the electrostatic work term corrections (based on total protein charges) are small and have not been included. It can be seen from Table XI that good agreement between observed and calculated rate constants is obtained. It is of interest that the rate constants for the reactions of azurin with other proteins appear to be in better agreement with the cross-relation than are the rate constants for its reactions with small metal complexes.

In concluding this section, it is useful to compare the self-exchange rate constant found for $\mathrm{Cyt}$ $c\left(1.2 \cdot 10^{3} \mathrm{M}^{-1} \cdot \mathrm{s}^{-1}\right)$ at $25^{\circ} \mathrm{C}$ with that found for azurin $\left(2 \cdot 10^{6} \mathrm{M}^{-1} \cdot \mathrm{s}^{-1}\right.$ at $50^{\circ} \mathrm{C}$ [276]). The 
TABLE XI

\section{COMPARISON OF OBSERVED AND CALCULATED RATE CONSTANTS FOR PROTEIN_PROTEIN REACTIONS}

Unless otherwise indicated, the self-exchange rate constants and reduction potentials are from Tables VII and VIII and the calculated rate constants do not contain the $W_{12}$ correction. The references in the last column should be consulted for the experimental rate constants.

\begin{tabular}{lllll}
\hline Oxidant & Reductant & $\begin{array}{l}k_{12 \text {,obsd }} \\
\left(\mathrm{M}^{-1} \cdot \mathrm{s}^{-1}\right)\end{array}$ & $\begin{array}{l}k_{12, \text { calcd }} \\
\left(\mathrm{M}^{-1} \cdot \mathrm{s}^{-1}\right)\end{array}$ & Ref. \\
\hline Cyt $c$ & Azurin & $1.6 \cdot 10^{3}$ & $3 \cdot 10^{4}$ & 284 \\
Plastocyanin & Cyt $c$ & $1.0 \cdot 10^{6}$ & $5 \cdot 10^{5} \mathrm{a}$ & 284 \\
Stellacyanin & Cyt $c$ & $3.5 \cdot 10^{2}$ & $4 \cdot 10^{3}$ & 283 \\
Azurin & Cyt $c-551$ & $6.0 \cdot 10^{6}$ & $8 \cdot 10^{5 \mathrm{~b}}$ & 284 \\
Plastocyanin & Cyt $c-551$ & $7.5 \cdot 10^{5}$ & $5 \cdot 10^{5 \mathrm{~b}}$ & 284 \\
Cyt $c$ & Cyt $c-551$ & $6.7 \cdot 10^{4}$ & $1 \cdot 10^{5 \mathrm{~b}}$ & 284
\end{tabular}

a The $W_{12}$ correction is considerably larger for this reaction than for the other systems considered, and was included in the calculation of $k_{12}$.

${ }^{b}$ Calculated using $1.2 \cdot 10^{7} \mathrm{M}^{-1} \cdot \mathrm{s}^{-1}$ for the self-exchange rate constant [206] and $+0.28 \mathrm{~V}$ for the reduction potential of the cytochrome $c-551$ couple [275]. The net charge on the protein is $-2 /-3$ [284].

activation energy of $54 \mathrm{~kJ} \cdot \mathrm{mol}^{-1}$ for the Cyt $c$ self-exchange [193] yields a $k$ of about $2 \cdot 10^{4}$ $\mathrm{M}^{-1} \cdot \mathrm{s}^{-1}$ at $50^{\circ} \mathrm{C}$. Oxidized and reduced azurin carry small net negative charges (Table $\mathrm{X}$ ) and hence have negligible coulombic repulsion, while ferro- and ferricytochrome $c$ have substantial positive charges (Table VII). This difference in coulombic repulsions contributes a factor of about 5 to the ratio of the rate constants. Again, the values of the electron-transfer distance $d$ differs for the two reactions. In the crystal of azurin from Alcaligenes denitrificans pairs of azurin molecules are related by an approximate 2 -fold axis, with their hydrophobic patches in contact and with a distance of $0.7 \mathrm{~nm}$ between the edges of the two histidine imidazole rings [285]. This distance is less than the $0.94 \mathrm{~nm}$ estimated earlier for the edge-toedge distance of the two histidines in the Cyt $c$ case. This difference is estimated to contribute a factor of $\exp (12 \cdot 0.24)$ or about 20 to the value of $k$. These three factors (temperature, coulombic repulsion and distance) account for the difference in self-exchange rates of Cyt $c$ and of azurin, if the values of $\lambda / 4$ for both are comparable. Interestingly, the geometry of the ligands in azurin is that of a distorted tetrahedron, intermediate between the tetrahedron common for 4-coordinate $\mathrm{Cu}^{\mathrm{I}}$ complexes and the square planar or tetrahedron common for 4-coordinate $\mathrm{Cu}^{\mathrm{II}}$, thereby reducing the reorganization term $(\lambda / 4)$ needed for electron transfer $[286,266]$.

\section{VG. Cytochrome c peroxidase}

We conclude this review with an example which illustrates the kinetic complexity which can arise in an oxidation-reduction scheme and for which an effort was made to extract a rate constant for an electron-transfer step. We use as an example the cytochrome $c$ peroxidase system, which catalyzes the oxidation of ferrocytochrome $c$ by hydrogen peroxide and alkyl hydroperoxides. The reaction of yeast cytochrome $c$ peroxidase $(\mathrm{CcP})$ with cytochrome $c$ [287-289] and with metal complexes involves several intermediates [290-292]. Scheme I summarizes some [291,293] (but not all [293]) of the features that have been proposed for the reaction of cytochrome $c$ with metal complexes. The first step is the formation of a CcP-peroxide complex $\mathrm{CcP}-\mathrm{OOH}$ which then undergoes a bimolecular reaction with the added reductant to form $\mathrm{RFe}^{\mathrm{IV}}$ or an intramolecular proton-catalyzed redox reaction to form $\mathrm{CcPI}$. The latter is described as an $\mathrm{Fe}^{\mathrm{IV}}$ (ferryl) species possessing an oxidized protein residue (methionine-172 or tryptophan-51) [292-294] ". There is some disagreement concerning the formulation of the subsequent steps indicated by the solid [291] vs. dashed [293] lines in 


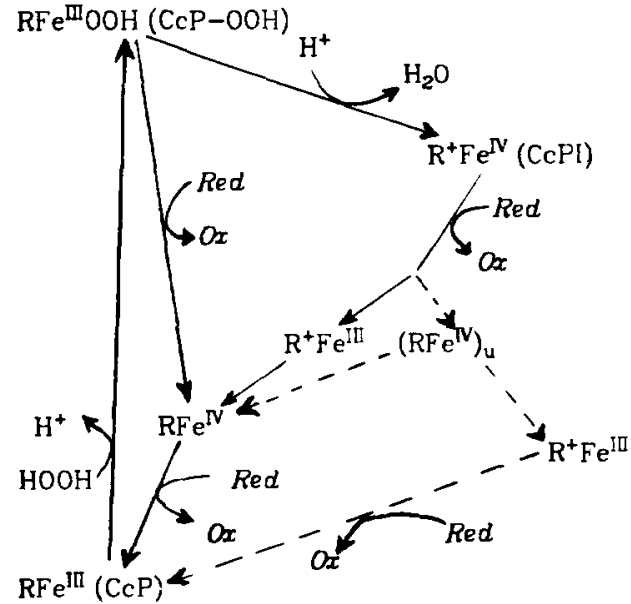

Scheme I.

the scheme, where $\left(\mathrm{RFe}^{\mathrm{IV}}\right)_{\mathrm{u}}$ denotes a species which is not in its most stable conformation.

The kinetics of the reactions of yeast cytochrome $c$ peroxidase with metal complexes has, so far, only been interpreted in terms of the mechanism indicated by the solid lines [291]. In this interpretation the rate law permits the assignment of a rate constant for the reduction of $\mathrm{R}^{+} \mathrm{Fe}^{\mathrm{IV}}$ to $\mathrm{R}^{+} \mathrm{Fe}^{\mathrm{III}}$ and/or for the reduction of $\mathrm{RFe}^{\mathrm{IV}}$ to $\mathrm{RFe}^{\mathrm{III}}$ (the kinetic data do not allow a unique assignment). Both reactions involve the reduction of the $\mathrm{Fe}^{\mathrm{IV}}$ peroxidase to the $\mathrm{Fe}^{\mathrm{III}}$ state and can be represented by $\mathrm{Fe}^{\mathrm{IV}} \mathrm{O}+e^{-}=\mathrm{Fe}^{\mathrm{III}} \mathrm{O}$. On the basis of the assumption that the stable form of the $\mathrm{Fe}^{\mathrm{III}}$ product is $\mathrm{Fe}^{\mathrm{III}} \mathrm{OH}$ (i.e., that the $\mathrm{Fe}^{\mathrm{III}}$ species contains a coordinated hydroxide ion), the $E^{0}$ for Eqn. 50 has been estimated to be $+0.7 \mathrm{~V}$ [291]. Application of the cross-relation without any work corrections then yields a rate constant of approx. $10^{2} \mathrm{M}^{-1} \cdot \mathrm{s}^{-1}$ for the exchange between the $\mathrm{Fe}^{\mathrm{IV}}$ and $\mathrm{Fe}^{\mathrm{III}}$ peroxidase. This value is higher by a factor of $1 \cdot 10^{6}$ than another estimate [290] in

\footnotetext{
IIt has recently been proposed (Ref. 295 and references cited therein) that the formation of CcPI from the resting state of the enzyme $\mathrm{CcP}\left(\mathrm{PFe}^{\mathrm{II}} \mathrm{R}\right)$ proceeds via an iron(IV) porphyrin cation-radical intermediate $\left(P^{+} \mathrm{Fe}^{\mathrm{IV}} \mathrm{R}\right)$ and that the imidazole coordinated to the iron center then mediates the oxidation of the nearby methionine-172 by the porphyrin radical to form $\mathrm{CcPI}\left(\mathrm{PFe}^{\mathrm{IV}} \mathrm{R}^{+}\right)$.
}

which proton-coupled changes were not considered $\$$. As discussed above, electron transfer through the exposed heme edge is implicated for the cytochrome $c$ system, and it is likely that a similar mechanism obtains for the peroxidase exchange as well [291].

The bimolecular rate constant for the reaction of ferrocytochrome $c$ with the peroxidase is approx. $5 \cdot 10^{8} \mathrm{M}^{-1} \cdot \mathrm{s}^{-1}$ at $\mathrm{pH} 6.0$ [287]. This very rapid rate, which is $10^{3}-10^{4}$-times more rapid than the reaction of the peroxidase with metal complexes of comparable redox potential and exchange rates, has been ascribed [291] to the stability of the precursor complex formed between cytochrome $c$ and the (negatively charged) peroxidase (binding constant, approx. $10^{5} \mathrm{M}^{-1}$ [45]). The X-ray structure of yeast cytochrome $c$ peroxidase has been determined [296] and computermodeling $[297,298]$ of the complex formed between cytochrome $c$ and the peroxidase implicates a structure in which the two heme groups are almost parallel with a perpendicular separation between their planes of $0.68 \mathrm{~nm}$ and a heme edge-to-edge separation of $1.65 \mathrm{~nm}$. Although coupling of the two heme groups via aromatic (specifically histidine-181 [299]) and conjugated groups has been invoked as an additional rate-enhancing factor [297,298], no 'special' electronic interaction of the reactants may be necessary to account for a first-order rate constant of approx. $10^{3}-10^{4} \mathrm{~s}^{-1}$. Thus $\Delta G^{*}$ is small ${ }^{\pi}$ and, for $\beta=12 \mathrm{~nm}^{-1}$ and $d=1.35 \mathrm{~nm}$ (after using the $0.3 \mathrm{~nm}$ correction in subsection IVB) the value of $1 \cdot 10^{13} \exp (-\beta d)$ equals $1 \cdot 10^{6} \mathrm{~s}^{-1}$.

\$The estimate of the self-exchange $k$ in Ref. 290 is based on a fitted redox potential $E^{0 \prime}=1.1 \mathrm{~V}$ for the CCPII $/ \mathrm{CPP}$ couple at $\mathrm{pH}=5.3$ and $\mu=0.1 \mathrm{M}$, where $\mathrm{CcPII}$ is the one-electron reduction product of $\mathrm{CCPI}$ : $E^{0 \prime}$ was deduced from applications of the cross-relation and based on deviations of the $f$ factor there from unity, a very uncertain procedure. The $E^{0}$, in [291] corrects the estimated redox potential for assumed $\mathrm{pH}$ effects and suffers from a similar uncertainty. Different assumptions about the nature of the proton transfers that are coupled to the electron transfer will lead to a different exchange rate.

1 The $\Delta G^{0}$, for the reaction has been estimated to be very favorable $[290,291]$ and $\lambda / 4$ for a heme-heme system is expected to be small. 


\section{Conclusions}

The theory of electron-transfer reactions has been particularly useful for correlating a wide body of experimental data on reactions of metal complexes in solution, particularly via the cross-relation. It has also been useful in understanding the absolute values of the reaction rates, and indeed gives reasonable agreement with the experimental data (Table I). It has been used for predictions, including that of the recently observed inverted effect. One large area of study, only briefly mentioned in this review, but which has played a significant role in the interplay of theory and experiment, has been that of electron transfers at electrodes. Here theory has been useful in predicting correlations between homogeneous and electrochemical electron transfers.

In the application to biological systems new features arise. Frequently, the reactants may be fixed some distance apart, and the attenuation of the coupling of their electronic orbitals with separation distance then becomes an additional significant factor; not infrequently the (edge-to-edge) separation distance of the redox centers is only approximately known. This additional factor is more readily studied when the reaction is in the 'barrierless' regime, i.e., in the regime where the driving force $-\Delta G^{0^{\prime}}$ has overcome the intrinsic reorganizational barrier $\lambda$.

There are several additional features which arise in the application of the theory to systems of biological interest for which increased information would be very useful. They include distances and orientations of reactants, and the reorganizational response of the protein to changes in charges of the redox centers. Nevertheless, the correlation between predicted and calculated rates for reactions involving redox proteins can be very good, as illustrated for the reactions of cytochrome $c$ with various inorganic reagents (Table VII). In the case of intramolecular electron transfers (subsection VC), the current results on biological systems are consistent with an electron-transfer rate that falls off exponentially with distance, with an exponent near those found for nonbiological systems (Table IV).

In applying the equations to the biological systems we have, for concreteness, estimated some of the numerical values of the rate-determining parameters even when they were not known independently. These estimates, made to illustrate the magnitudes of the parameters, are of course to be regarded as tentative at this time. Nevertheless, it is encouraging that the values used lead to a consistent treatment of the reactions of the metalloproteins (mainly cytochrome $c$ ) with a variety of substrates and of reactions in the photosynthetic system.

\section{Appendix I. Expressions for activation- and diffu-} sion-controlled reaction rates

\section{IA. No steric effects}

The physical events involved in a bimolecular collision in solution between two molecules $A$ and $B$ involve a random diffusion of the molecules toward and away from each other, becoming in some instances a close-contact encounter complex A $\cdots$ B and then diffusing apart. We may write this process as

$$
\mathrm{A}+\mathrm{B} \underset{k_{-\mathrm{D}}}{\stackrel{k_{\mathrm{D}}}{\rightleftharpoons}} \mathrm{A} \cdots \mathrm{B}
$$

The electron transfer can occur at any stage of this process, with shorter distances weighted more favorably because of the nonadiabatic $\exp (-\beta r)$ factor. In the absence of reaction, the rate constant for diffusion $k_{\mathrm{D}}$ multiplied by the average number of repeated encounters $\gamma$ will equal the mean collision frequency $Z$ of the pair in solution. Since $k_{\mathrm{D}}$ for small uncharged species with no specific interactions is of the order of $1 / 10$ to $1 / 100$ the value of $Z, \gamma$ is of the order of 10 to 100 for such systems.

If during the encounters, electron transfer occurs with a first-order rate constant $k_{1}$, and if the reverse electron transfer rate is negligible relative to the rate of separation of $\mathrm{A}^{+}$and $\mathrm{B}^{-}$,

$\mathrm{A} \cdot \mathrm{B} \stackrel{k_{1}}{\rightleftharpoons} \mathrm{A}^{+} \mathrm{B}^{-} \rightarrow \mathrm{A}^{+}+\mathrm{B}^{-}$,

the overall rate constant for electron transfer is given by Eqn. 53 in a steady state approximation 
for the concentration of $\mathrm{A} \cdots \mathrm{B}[300,301]$, for the case of spherical reactants:

$\frac{1}{k_{\mathrm{obsd}}}=\frac{1}{k_{\mathrm{D}}}+\frac{1}{K_{\mathrm{A}} k_{1}}$,

where $K_{\mathrm{A}}$, which equals $k_{\mathrm{D}} / k_{-\mathrm{D}}$, is the equilibrium constant for formation of $\mathrm{A} \cdots \mathrm{B}$ in Eqn. 51. The force field used to calculate $K_{\mathrm{A}}$ and also $k_{\mathrm{D}}$ (both depend on long-range electrostatic forces) is taken to be spherically symmetric. The value of $k_{\mathrm{D}}$ is given by [302]:

$k_{\mathrm{D}}=4 \pi D / \int_{r=\sigma_{12}}^{\infty} \exp \left(w^{\mathrm{r}} / R T\right) r^{-2} \mathrm{~d} r$

where $D$ is the sum of the diffusion constants of the two reactants and the work term $w^{\mathrm{r}}$ is a function of $r$ and, in the presence of an ion atmosphere, is assumed to be given by its equilibrium value, e.g., by Eqn. 25 in a Debye-Hückel approximation. (If the ionic atmosphere were not quite keeping pace with these diffusing reactants, a different effective $w^{\mathbf{r}}$ would be needed.)

The rate constant $k$ appearing in Eqn. 1 is the same as the $K_{\mathrm{A}} k_{1}$ in Eqn. 53a. In other words, when the reaction is partially diffusion-controlled, Eqn. 53a is used with $K_{\mathrm{A}} k_{1}$ given by Eqn. 1, and hence by

$K_{\mathrm{A}} k_{1}=\kappa A \sigma^{2} \exp \left(-\Delta G^{*} / R T\right)$.

Several models for $A \sigma^{2}$ are available. For example, since $K_{\mathrm{A}} k_{1}$ is equal to the rate constant when diffusion control is absent, one sees that it is equivalent to some mean (equilibrium) collision frequency in solution multiplied by the reaction efficiency. The effective collision frequency in solution is the value $Z_{\text {soln }}$ in the absence of the work term $w^{\mathrm{r}}$, multiplied by an exponential factor $\exp \left(-w^{\mathrm{r}} / R T\right)$, which influences the probability of close encounters. The reaction efficiency is $\kappa$ $\exp \left(-\Delta G_{r}^{*} / R T\right)$, where $\Delta G_{r}^{*}$ is the free energy barrier to reaction between fixed sites at the separation distance $r$ and is given by Eqn. 32 . Thereby:

$$
\begin{gathered}
\kappa A \sigma^{2} \exp \left(-\Delta G^{*} / R T\right)=\kappa Z_{\text {soln }} \\
\quad \times \exp \left[-\left(w^{\mathrm{r}}+\Delta G_{r}^{*}\right) / R T\right]
\end{gathered}
$$

If $Z_{\text {soln }}$ has its gas phase value $(8 \pi R T / m)^{1 / 2} \sigma^{2}$, where $m$ is the reduced mass of the collision partners $m_{\mathrm{A}} m_{\mathrm{B}} /\left(m_{\mathrm{A}}+m_{\mathrm{B}}\right)$ and $\sigma$ is the separation distance $r$ in the encounter complex $\mathbf{A} \cdots \mathbf{B}$, then, since $\Delta G^{*}$ equals $\left(w^{\mathrm{r}}+\Delta G_{r}^{*}\right)$,

$A=(8 \pi R T / m)^{1 / 2}$

Another model $[18,21,303]$ is to take the equilibrium constant $K_{\mathrm{A}}$ as the effective volume occupied by the separation coordinate over which reaction occurs, $4 \pi r^{2} \delta r$ (the $r$ 's over which electron transfer can still occur significantly lie between $r$ and $r+\delta r) ~ "$, multiplied by the additional free energy factor $\exp \left(-w^{\mathrm{r}} / R T\right)$ describing the additional probability of finding the pair in the reaction zone:

$K_{\mathrm{A}}=4 \pi \sigma^{2} \delta \sigma \exp \left(-w^{\mathrm{r}} / R T\right)$,

where $r$ has been set equal to $\sigma$.

The first-order rate constant $k_{1}$ in reaction 52 is taken to be an effective frequency $\nu$ for motion along a 'reaction coordinate' (vibrational mainly, but also with solvational contributions) multiplied by the reaction efficiency, mentioned earlier $\kappa$ $\exp \left(-\Delta G_{r}^{*} / R T\right)$

$k_{1}=\kappa \nu \exp \left(-\Delta G_{r}^{*} / R T\right)$.

We then have from Eqns. 54, 57 and 58 and the relation between $\Delta G^{*}$ and $\Delta G_{r}^{*}$ that

$A=4 \pi \nu \delta \sigma$

Eqns. 56 and 59 yield a value for $A$ within a factor of 10 or so of each other, depending, of course, on the size of $\delta \sigma$ which is typically of the order of $1 / \beta$ or $0.08 \mathrm{~nm}[21,304]$. This is the value used in the calculations in the present paper.

In the case of an adiabatic reaction $\kappa$ is unity and the frequency $\nu$ in Eqn. 58 is given as a

\footnotetext{
This $\delta r$ also appears in the theory described in Refs. 3 and 58 , via a term $\rho$ there which is estimated to be of the order of unity and hence omitted from the present Eqn. $1 ; \rho$ is the ratio of this $\delta r$ to the width of the 'equivalent equilibrium distribution' along the reaction coordinate (defined in Refs. 2 and 3).
} 
weighted sum of nuclear frequencies $\nu_{i}$, namely:

$\kappa \nu=\nu=\left(\frac{\Sigma_{j} \lambda_{j} \nu_{j}^{2}}{\Sigma_{j} \lambda_{j}}\right)^{1 / 2} \quad$ (adiabatic case)

when there are several vibrational motions contributing to $\nu$ and to $\lambda\left(\lambda=\Sigma_{j} \lambda_{j}\right)$ [305]. In the case of a nonadiabatic reaction $(\kappa \ll 1)$ the product $\kappa \nu$ is given instead by Eqn. 32, and is seen to be independent of the frequencies $\nu_{j}$ of the nuclear motion!

An estimate of the value of $\kappa \nu$ at contact of the reactants can be made from values of $H_{\mathrm{AB}}$ in the literature. The estimate of $H_{\mathrm{AB}}$ in Ref. 63 for edge-to-edge contact of the hemes and $\lambda \cong 1 \mathrm{~V}$ yields $\kappa \nu \approx 3 \cdot 10^{12} \mathrm{~s}^{-1}$, which is close to the value of $10^{13} \mathrm{~s}^{-1}$ assumed in Section V. An estimate of $H_{\mathrm{AB}}$ in Ref. 306 for a face-to-face contact of hemes gives a value for $\kappa \nu$ even larger than $1 \cdot 10^{13}$ $\mathrm{s}^{-1}$. Since $\kappa \nu$ shouldn't exceed the value for an adiabatic reaction, and the latter equals a typical vibration frequency approx. $10^{13} \mathrm{~s}^{-1}$, one concludes that $\kappa \approx 1$ for the latter contact.

We next give the equation for ionic strength effects obtained from Eqns. 1-3 by including the effects on the work terms (Eqn. 25) and on the activity coefficients affecting the $\Delta G^{0^{\prime}}$ in the prevailing medium. Using the expression given in the footnote to Eqn. 38 for small $\left|\Delta G^{0^{\prime}} / \lambda\right|$, and using the Debye-Hückel expression for the activity coefficients, we obtain Eqn. 61 for the case of reaction 7. In this reaction $z_{1}$ increases by unity and $z_{2}$ decreases by unity in a one-electron transfer:

$$
\begin{aligned}
\ln k= & \ln \bar{k}+\left[\left(z_{1}+\frac{1}{2}\right)\left(z_{2}-\frac{1}{2}\right)-\frac{1}{4}\right] C \\
& +\frac{N \mathrm{e}^{2} B \sqrt{\mu}}{D_{\mathrm{s}} R T}\left(\frac{\left(z_{1}+\frac{1}{2}\right)}{1+B \sigma_{1} \sqrt{\mu}}-\frac{\left(z_{2}-\frac{1}{2}\right)}{1+B \sigma_{2} \sqrt{\mu}}\right)
\end{aligned}
$$

where $C$ is the factor which multiplies $-z_{1} z_{2}$ in Eqn. 38. In a self-exchange reaction $z_{1}+1$ equals $z_{2}$, and $\sigma_{1}$ equals $\sigma_{2}$, so that the last term in Eqn. 61 then vanishes. For any reaction Eqn. 61 reduces to Eqn. 41 at sufficiently low $\mu$. (To obtain the latter one notes that in Eqn. $25 \sigma_{12}=\frac{1}{2}\left(\sigma_{1}+\sigma_{2}\right)$.)

\section{IB. Steric effects included}

A steric factor $S$, not present in the above equations, was introduced in subsection VB to take into account the fact that only some limited range of orientations of $\mathrm{A}$ and $\mathrm{B}$ at a given $r$ may be appropriate for electron transfer in protein systems. We denote by $A-B$ and $A B$ pair in such a properly oriented configuration; $A-B$ is formed from the encounter complex $A \cdots B$ by suitable reorientation of $\mathrm{A}$ and/or $\mathrm{B}$ :

$$
\mathrm{A} \cdot \mathrm{B} \rightleftharpoons \mathrm{A}-\mathrm{B} \rightarrow \mathrm{A}^{+}+\mathrm{B}^{-}
$$

For the present review it is convenient to separate approximately two contributions to the equilibrium constant (denoted by $K_{e}$ ) for the formation of A-B from A and B. This constant $K_{e}$ differs from that for forming $A \cdots B$ from $A$ and $B$ in two respects. (1) The work term appearing in $K_{\mathrm{A}}$ (Eqn. 57) is now for a fixed orientation of $\mathrm{A}$ and $B$ and is based on the actual charge distribution in the transition state rather than on total charges; (2) There is a steric factor $S$ to correct for the fact that the $4 \pi$ in $K_{\mathrm{A}}$ assumes. spherical symmetry, i.e., it is obtained by integrating uniformly over the angular coordinates. This factoring of the equilibrium constant $K_{\mathrm{e}}$ into $S$ and $K_{\mathrm{A}}$ is, as already noted, approximate ".

A first-order rate constant $k_{1}$ for a properly docked pair (active sites in contact) can be defined as in Eqn. 58, where $\Delta G_{r}^{*}$ is given by Eqn. 32 . However, now the $w^{\mathrm{r}}$ and $w^{\mathrm{p}}$ appearing in $\Delta G^{0}$ there refer to work terms for forming $\mathrm{A}-\mathrm{B}$ and $\mathrm{A}^{+}-\mathrm{B}^{-}$in the properly docked configuration (the same for both). The (activation-controlled) bi-

\footnotetext{
Thile the factoring of $K_{\mathrm{e}}$ can be avoided by using statistical mechanics, the resulting expression would be more complicated. The $K_{\mathrm{e}}$ that is used above ( $S K_{\mathrm{A}}$ ) actually has three contributions to the formation of $\mathrm{A}-\mathrm{B}$ from $\mathrm{A}$ and $\mathrm{B}$, a geometrical one in $K_{\mathrm{A}}$ (the $4 \pi \sigma^{2} \delta \sigma$ ), a nongeometrical one $\left[\exp \left(-w^{\mathrm{r}} / R T\right)\right]$ and a reorienting-into-a-docking one $S$. In the more rigorous statistical mechanical expression all contribute to a single free-energy expression $W^{\mathrm{r}}, K_{\mathrm{e}}=$ $\exp \left(-W^{\mathrm{r}} / R T\right)$, though in a complicated, entangled way. The quantity $W^{\mathrm{r}}$ is a work term which includes the geometrical localization of A and B into a small range of $r$ 's, the localization of their relative angular coordinates into an interval, and the electrostatic and other nonelectrostatic contributions. In contrast, the $w^{\mathbf{r}}$ in Eqn. 57 is only one of these.
} 
molecular rate constant can be written as $S K_{\mathrm{A}} k_{1}$ with $K_{\mathrm{A}}$ given by Eqn. 57, where $w^{\mathrm{r}}$ is now for the docked configuration. Hence,

$k=S K_{\mathrm{A}} \kappa \nu \exp \left(-\Delta G_{r}^{*} / R T\right)$.

Eqns. 43 and 44 immediately follow from Eqns. 63 and 32. Further, the maximum value of the activation-controlled $k$ is obtained by setting $\Delta G_{r}^{*}$ equal to zero in Eqn. 63, and yields the value given in subsection $\mathrm{VC}, S K_{\mathrm{A}} \kappa \nu$.

An experimental value for $k_{1}$ can be obtained from the bimolecular rate constant $k$ and the equilibrium constant $K_{\mathrm{e}}$ (measured kinetically or spectroscopically) for formation of $A-B$ from $A$ and $\mathrm{B}$, if the $1: 1$ complex principally exists as $\mathrm{A}-\mathrm{B}$ rather than $\mathrm{A} \cdots \mathrm{B}$, namely $k_{1}=k / K_{\mathrm{e}}$. If $K_{\mathrm{e}}$ is not known, its calculated value $S K_{\mathrm{A}}$ may be used instead, and we have $k_{1}=k / S K_{\mathrm{A}}$. Both results are used in subsection VC.

Since the work terms in Eqn. 63, namely, the $w^{\mathrm{r}}$ and $w^{\mathrm{p}}$ in $K_{\mathrm{A}}$ and $\Delta G_{r}^{*}$, are appropriate to the docked configuration, they require a detailed knowledge of the charge distribution, particularly in the vicinity of the active sites of the proteins. Simpler calculations, discussed in subsection IVD, are typically adopted and one of them is used here, for convenience, in the form of Eqn. 25. It should be noted, however, that the binding constants for natural partners, which generally have opposite charges, tend to be considerably higher than the values calculated from Eqns. 25 and 57, presumably because of the breakdown of the Debye-Hückel type theory for such cases, a result anticipated by Bronsted in his theory of specific ionic interaction [176] (see subsection VE). In the cases discussed in this article, the actual binding constants were used for these cases.

When the rate constant $k$ given by. Eqn. 63 becomes sufficiently high the reaction may, as in the spherically symmetric case (Appendix IA), become diffusion-controlled. Whereas the problem in the latter case involved the solution of a differential equation for diffusion and reaction, leading in the steady state to Eqn. 53, the differential equation to be solved now also involves a rotationaldiffusive process in the reactants. For simplicity, we shall continue to assume Eqn. 53, with $K_{\mathrm{A}} k_{1}$ now given by the $k$ in Eqn. 63. If rotational diffusion were sufficiently slow, however, the maximum rate might now be below $k_{\mathrm{D}}$, even if $k_{\mathrm{D}} \gg$ $K_{\mathrm{A}} k_{1}$. However, the rate constants for the plastocyanin reactions show that for these systems the rate constant is close to the translational diffusion-controlled rate constant $k_{\mathrm{D}}$.

We consider, for completeness, rate saturation effects which sometimes occur. We examine first reactions 51 and 52. In Eqn. 53 it was tacitly assumed that $(\mathrm{A} \cdot \mathrm{B}) \ll(\mathrm{A})$. Under these conditions a steady-state assumption for $(A \cdots B)$ is appropriate. More generally the reaction is biphasic in time with a fast time constant being associated with the establishment of a near steady-state for $(\mathrm{A} \cdots \mathrm{B})$ and with the slow time constant being given by

$\frac{1}{k_{\text {obsd }}}=\frac{1}{K_{\mathrm{A}} k_{1}(\mathrm{~B})}+\frac{1}{k_{\mathrm{D}}(\mathrm{B})}+\frac{1}{k_{1}}$,

where $k_{\text {obsd }}$ is measured in $\mathrm{s}^{-1}$. When (B) becomes very small the $1 / k_{1}$ can be neglected and Eqn. 64 reduces to Eqn. 53. On the other hand, when (B) becomes large, $k_{\text {obsd }}$, measured in $\mathrm{s}^{-1}$, becomes $k_{1}$ and, thereby, independent of (B). Rate saturation has occurred.

Rate saturation effects may also occur in a reaction mechanism involving the formation of a dead-end complex. The slow step can now even be the dissociation of the dead-end complex rather than the electron transfer. Some discussion of these effects is given in Refs. 197 and 281, and is omitted here in the interest of brevity.

\section{IC. Dependence of $H_{A B}$ on nuclear configuration}

In the treatment in Section II, it was assumed that the thermal electron-transfer matrix element $H_{\mathrm{AB}}$ was independent of nuclear configuration (the Condon approximation). Actually, there may be some dependence, e.g., Ref. 307. The detailed analysis of this effect depends, in part, on whether the nuclear motion is largely classical (minor nuclear tunneling) or highly quantum mechanical (extensive nuclear tunneling).

For the present purpose a very simple treatment is adopted in which the nuclear motion is treated classically, so that the electron transfer occurs at 
the intersection of the curves in Fig. 1". It can then be shown ${ }^{\S}$ that for a system at the intersection the potential energy of the reductant minus its potential energy after loss of an electron, equals $\left(-\lambda_{1} \Delta G^{0^{\prime}} / \lambda\right)$, where $\lambda_{1}$ is the contribution of the reductant (reactant 1$)$ to $\lambda\left(\lambda=\lambda_{1}+\lambda_{2}\right)$, and that the corresponding value is $\lambda_{1}$ when the reactant is in its equilibrium configuration. Thus, if for reactant 1 in its equilibrium configuration the vertical ionization potential for donation of an electron to an adjacent bridging unit is $V_{0}^{\mathrm{e}}$, the corresponding value $V_{0}$, when the system is at the intersection is

$V_{0}=V_{0}^{e}-\frac{\lambda_{1} \Delta G^{0^{\prime}}}{\lambda}-\lambda_{1}$

Hence, also, $V_{0}$ equals $V_{0}^{e}$ when $-\Delta G^{0 \prime}=\lambda$. If $\Delta G^{0^{\prime}}$ is varied at fixed $\lambda_{1}$ and $\lambda_{2}$ by varying only the oxidant, $V_{0}$ changes only because the energy of the intersection in Fig. 1 changes. The magnitude

In a nuclear tunneling situation, a broader interval of coordinates is involved for tunneling from any initial quantum state of the reactants.

$\S$ The argument used is based, for the present purpose, on a harmonic oscillator analysis and, to simplify the discussion, allots a solvent contribution outside the coordination shell to each ion, so that $\lambda=\lambda_{1}+\lambda_{2}$. (A more detailed treatment of the solvent would not pose any difficulty and would be based on arguments related to those used in Ref. 12.) The potential energy in coordinate $q$ of the reactant, specifically the reductant, is $f\left(q-q^{\mathrm{r}}\right)^{2} / 2$, where $q^{\mathrm{r}}$ is the equilibrium value of $q$ and $f$ is a force constant, assumed here to be the same in the ionized and ground states. If the electron is suddenly lost, this potential energy of this reactant is now $f\left(q-q^{\mathrm{P}}\right)^{2} / 2$, where $q^{p}$ is the new equilibrium value of $q$ after the electron is lost. Setting $q=q^{r}$, the vertical ionization energy $V_{0}$ for an ion in its equilibrium nuclear configuration is seen to contain a term $f\left(q^{\mathrm{r}}-q^{\mathrm{p}}\right)^{2} / 2$, making it less than it would otherwise be. Summed over all $q^{\text {'s }}$ of the reductant this term $\Sigma f\left(q^{r}-\right.$ $\left.q^{p}\right)^{2} / 2$ is denoted by $\lambda_{1}$. We next make a similar analysis when the reductant is in a configuration at the intersection in Fig. 1. A treatment in Ref. 308 is used. In particular, at the intersection we note that a coordinate $q$ equals $q^{*}$, namely $(m+1) q^{r}-m q^{p}$ [308]. The contribution to the vertical ionization potential is now $\Sigma f\left(q^{*}-q^{\mathrm{p}}\right)^{2} / 2-\Sigma f\left(q^{*}\right.$ $\left.-q^{\mathrm{r}}\right)^{2} / 2$, which equals $(2 m+1) \Sigma f\left(q^{\mathrm{r}}-q^{\mathrm{P}}\right)^{2} / 2$, i.e., $(2 m+$ 1) $\lambda_{1}$. But $2 m+1$ equals $-\Delta G^{0 \prime} / \lambda$ [308], and so one obtains the result cited in the text. of the thermal matrix element $\left|H_{\mathrm{AB}}\right|$ is given by

$\left|H_{\mathrm{AB}}\right|=B\left(\frac{B}{V_{0}}\right)^{N}$

where $V_{0}$ is given by Eqn. $65, N$ is the number of intervening sites (identical bridging units) between the two reactants and $B$ is the exchange integral between adjacent sites [307,309-312] I. ( $B$ was taken here for simplicity to be the same for all adjacent pairs.) Thereby, the $\beta$ in Eqn. 37 is given by:

$\beta=2 l^{-1} \ln \frac{V_{0}}{B}$

where $l$ is the length of a bridging unit.

If, instead of varying the oxidant, the reductant were varied at fixed $\lambda_{1}, \lambda_{2}$ and $\Delta S^{0^{\prime}}$, and if $V_{0}^{\text {e }}$ again denotes the value of $V_{0}$ for the case where $\Delta G^{0^{\prime}}=-\lambda$, the value of $V_{0}$ at any other $\Delta G^{0^{\prime}}$ would now be given by Eqn. 68 . A change in $\Delta G^{0^{\prime}}$ now changes $V_{0}$ not only by the nuclear configuration term but also by changing the electronic energy level of the reductant itself.

$V_{0}=V_{0}^{\mathrm{e}}-\frac{\lambda_{1} \Delta G^{0^{\prime}}}{\lambda}-\lambda_{1}-\left(\Delta G^{0^{\prime}}+\lambda\right)$

We next apply Eqns. 65 and 67 to experiments in which $-\Delta G^{0^{\prime}}$ is varied from 0 to $\lambda$ in the normal region and from $\lambda$ to $2 \lambda$ in the abnormal region by varying the oxidant (as was the case in the experiments in Ref. 36.) For concreteness, we treat a solvent-mediated electron transfer and take $2 \lambda_{1}=\lambda=1 \mathrm{eV}$ and $V_{0}^{\mathrm{e}}=5 \mathrm{eV}$, the value for a vertical low energy ion-to-solvent charge-transfer transition. The change in $\beta$ when $\left|\Delta G^{0^{\prime}}\right|$ is changed by $\lambda$ is estimated from Eqn. 67 to be about 0.4 $\mathrm{nm}^{-1}$. If the separation distance $d$ is $1.0 \mathrm{~nm}$, then $\beta d$ changes by about 0.4 when $\left|\Delta G^{0^{\prime}}\right|$ changes by $\lambda$, while the classical free-energy barrier in the

\footnotetext{
The superexchange mechanism via an atom was considered earlier in Ref. 313. The expression for the typical case that there are several orbitals in a bridge involves a multiple sum over these orbitals for each bridge, and each $B / V_{0}$ depends on the orbital. See, for example, the footnote in subsection IVA and Eqn. 25 of Ref. 73, and the extension of the latter to more than two bridges [73].
} 
expression $(\lambda / 4 R T)\left(1+\Delta G^{0^{\prime}} / \lambda\right)^{2}$ at room temperature equals $9.6,0$ and 9.6 when $-\Delta G^{0^{\prime}}=0, \lambda$ and $2 \lambda$, respectively. The effect of variation of $\beta$ in the normal region or in the abnormal region, while not negligible, is seen to be minor in comparison with the variation in $(\lambda / 4 R T)(1+$ $\left.\Delta G^{0^{\prime}} / \lambda\right)^{2 \S}$. To the extent that nuclear tunneling is important, the effective sampling of extremes of potential energy and the thermal barrier would both be less.

\section{ID. Miscellaneous}

Eqn. 53, or its equivalent, was originally derived by solving the diffusion equation subject to the boundary condition that reaction occurs with a certain efficiency at the boundary $r=\sigma$. Recently, this diffusion equation, or really a reaction-diffusion equation, was solved numerically for the case that reaction can occur at any $r$ with a reaction probability which varies as $\exp (-\beta r)$ [55]. The results showed that within this 'continuum' framework for diffusion, Eqn. 53 provides a good approximation for the conditions studied, e.g., as in Table V of Ref. 55.

Not considered in the present review is the effect of the dynamics of solvent dielectric relaxation on electron-transfer rates, experimental data on this subject being almost absent. A recent experimental study on an intramolecular electron transfer in the barrierless regime has shown agreement between the rate constant of the electron transfer and the reciprocal of a ('constant-charge' corrected) longest measured dielectric relaxation time [314]. Several recent theoretical studies on solvent dynamics in charge-transfer reactions include references (Refs. 315 and 316, and references cited therein; also Sumi, H. and Marcus, R.A., unpublished data).

We conclude this section with some comments on the slope $\alpha$ of plots of $-R T \ln k$ vs $\Delta G^{0^{\prime}}$ at $\Delta G^{0^{\prime}}=0$, or, in the electrochemical case, versus the activation overpotential $\eta$ at $\eta=0$. It follows

$\$$ Use of the square-barrier model for $\beta$ mentioned in subsection IVC would be more artificial and yields a $\beta$ varying as $V_{0}^{1 / 2}$ instead of as $\ln V_{0}$. Also, hole transfer might occur, but for brevity of presentation only electron transfer is considered. from the analysis given in Eqns. A-14, 67 and A-11 of Ref. 3 that this $\alpha$ at $\Delta G^{0^{\prime}}=0$ or $\eta=0$ is given in the classical treatment by

$\alpha=\frac{1}{2}+\frac{\lambda_{\mathrm{i}}}{4 \lambda} \sum_{s} \frac{f_{s}^{\mathrm{r}}-f^{\mathrm{r}} p_{s}}{f_{s}^{\mathrm{r}}+f_{s}^{\mathrm{P}}} \frac{\lambda_{s i}}{\lambda}$,

where $\lambda_{i}$ is the vibrational ('inner-coordination shell') contribution to $\lambda$ (only the force constants $f$ in the inner-coordination shell were assumed to change) and where $\lambda_{s i}$ is the contribution of coordinate $q_{s}$ to $\lambda$.

We apply Eqn. 69 next to consider one model that has recently been assumed [43] for the solvation of singly charged organic ions. In this model the organic ion-solvent interaction is represented by a harmonic term with a force constant (assumed to be the same for cations as for anions) which exceeds considerably the force constant for the interaction of the solvent with an uncharged organic molecule [43]. Such a model clearly does not obey the quadratic free-energy relation used in obtaining Eqns. 1-3 and would predict from Eqn. 69 that the slope of a $-R T \ln k$ vs. $\Delta G^{0^{\prime}}$ plot at $\Delta G^{0^{\prime}}=0$ would be quite different from 0.5 for reactions between uncharged molecules to form ions, such as $A+B \rightarrow A^{+}+B^{-}$or for the reverse reaction. (In reactions such as $\mathrm{A}^{+}+\mathrm{B} \rightarrow \mathrm{A}+\mathrm{B}^{+}$ or $A+B^{-} \rightarrow A^{-}+B$, there is a compensating effect in Eqn. 69, so even quite different force constants $f_{s}^{\mathrm{r}}$ and $f_{s}^{\mathrm{P}}$ can lead [3] to a slope near 0.5.) Similar remarks apply to electrochemical oxidations and reductions at $\eta=0$.

The above result, namely of deviations from 0.5 arising from the model in Ref. 43 can be shown by adapting Eqn. 69 so as to include that model. There were only the ion-solvent coordinates in Ref. 43, and we will write $\lambda=\lambda_{i}$. Using the model the last term in Eqn. 69 then becomes $\frac{1}{4}\left(f^{\mathrm{r}}-\right.$ $\left.f^{\mathrm{p}}\right) /\left(f^{\mathrm{r}}+f^{\mathrm{p}}\right)$. The value of $f^{\mathrm{r}} /\left(f^{\mathrm{p}}-f^{\mathrm{r}}\right)$ assumed in Ref. 43 for a reaction such as $A^{*}+B \rightarrow$ $\mathrm{A}^{-}+\mathrm{B}^{+}$was between 0.1 and $0.3 \%$. It yields an $\alpha$,

\footnotetext{
" However, the treatment in Refs. 318 and 43 differed from the present one. It was classical for the ion-solvent coordinate and quantum for the other modes in Ref. 318 while in Ref. 43 it was 'semiclassical' in the sense $[63,64]$ discussed in subsection IIB for the ion-solvent coordinate with a high $(800$ $\mathrm{cm}^{-1}$ ) assumed frequency for that coordinate and with no other modes.
} 
based on Eqn. 69, of between 0.66 and 0.71 for such a homogeneous reaction or, in an electrochemical reaction, for the formation of an ion from an uncharged molecule. (It yields a value of $\alpha$ between 0.34 and 0.29 for the reverse reaction.) These $\alpha$ 's differ considerably from the experimental $\alpha$ 's (approx. 0.5 ) given in Table III at $\Delta G^{0^{\prime}}=0$ for the organic compounds and differ from the typical values of approx. 0.5 at $\eta=0$ for the electrochemical reduction or oxidation of organic molecules [319-325]

The solvent model proposed in Ref. 43 was introduced to explain why the inverted effect was not found [326] for the intermolecular electron transfer $\mathrm{A}^{*}+\mathrm{B} \rightarrow \mathrm{A}^{ \pm}+\mathrm{B}^{\mp}$. Recently, however, the inverted effect was found for the intramolecular $\mathrm{A}^{*}-\mathrm{B} \rightarrow \mathrm{A}^{+}-\mathrm{B}^{-}$reaction [38], complementing the recent intramolecular and frozen-solvent observations of the inverted effect $[36,37]$ for reactions of the type $\mathrm{A}^{-}-\mathrm{B} \rightarrow \mathrm{A}-\mathrm{B}^{-}$. Moreover, the bridges thus introduced are not expected to have. any significant effect on the previously postulated $[43,318]$ specific ion-solvent forces. All of the results suggest that the explanation for the lack of the inverted effect in Ref. 326 lies in some direction other than specific ion-solvent forces. For example, the quenching of the fluorescence of $A^{*}$ may arise not only from an electron transfer, but also (particularly if the electron transfer becomes slow) by competitive pathways, such as exciplex formation followed by a radiationless transition (e.g., Ref. 55 or references cited in Ref. 318). A variety of other possibilities exist [55]. However, a further analysis of the incidence and consequences of specific ion-solvent interactions would be desirable, as well as further pursuit of intramolecular examples of reactions such as $A^{*}-B \rightarrow A^{+}-B^{-}$.

\section{Appendix II. Franck-Condon factors and absolute rates}

The calculation of Franck-Condon factors has been discussed by many authors, both for radia-

\footnotetext{
$\$$ Numerous electrochemical results on metal complexes are also available and typically have slopes of about 0.5 at $\eta=0$ $[132,133]$. The theoretical value for these metal complexes given by Eqn. 69 differs slightly (approx. 0.04) from 0.5 when typical differences of vibrational force constants in the
}

tionless transitions and for electron transfers. Of interest in the present case are the calculations for the case where many coordinates are present (the environment). For a reaction proceeding from the lowest state of the reactant(s) to yield quantum states of a set of $N$ degenerate vibrational modes of the product(s), each having the same frequency $\nu$, the rate constant is found from Eqn. 13 to be given by ${ }^{\pi}$ :

$k=\frac{2 \pi}{\hbar} H_{\mathrm{AB}}^{2} \sum_{v=0}^{\infty} \frac{\mathrm{e}^{-S} S^{v}}{\Gamma(v+1)} \delta\left(\Delta E^{0^{\prime}}+v h \nu\right)$.

In Eqn. $70, \nu$ is assumed to be the same for the products as for the reactants. The $\lambda$ appearing in $S(S=\lambda / h \nu)$ is $N$-times the value of $\lambda$ for an individual vibrational mode; $\delta$ is the Dirac delta function; $v$ is the principal quantum number for the degenerate states of the $N$ vibrationally-excited modes. Eqn. 70 is intended to be an approximation to the actual situation where the frequencies of the vibrational modes are not equal. In the latter case, there are many largely nondegenerate levels thermally occupied rather than only a relatively small number of highly degenerate levels. The sum in Eqn. 70 can then be replaced by an integral over $v$, and, on making use of the delta function, Eqn. 71 is then obtained:

$k=\frac{2 \pi}{\hbar} H_{\mathrm{AB}}^{2} \frac{\mathrm{e}^{-S} S^{v}}{h \nu \Gamma(v+1)}$,

where $\nu$ is a mean frequency (defined for example in Ref. 327); $v$ now denotes $-\Delta E^{0^{\prime}} / h \nu$ and need no longer be an integer. Since any change in $\nu$ is

oxidized and reduced forms of the reactant are taken into account [3].

I The Franck-Condon factors which appear in Eqn. 13 are obtained, for example, in Ref. 327, Eqn. 9.16. This reference also contains the idea of using a mean frequency when there are a number of contributing vibrational modes. One derivation of the equations for the case of one coordinate is given in Ref. 328 for a spectral band shape. The Franck-Condon factor in Eqn. 70 is obtained by setting $h \nu=0$ and $h \nu_{\mathrm{ab}}=$ $\Delta E^{0 \prime}$ in Eqn. 4-115 of Ref. 328. Eqn. 70 is related to Eqn. 19, or really to a predecessor of that equation, namely, Eqn. 5 of Ref. 27. When the latter is used and the limit $\lambda_{0} \rightarrow 0$ is introduced, one obtains Eqn. 70. (One uses the fact that when $a \rightarrow 0,(1 / a \sqrt{\pi}) \exp \left(-x^{2} / a^{2}\right)$ becomes $\delta(x)$.) However, Eqn. 70 can be derived more directly, as in Ref. 328 . 
neglected, as is any other contribution to $\Delta S^{0^{\prime}}$, this $v$ also equals $-\Delta G^{0^{\prime}} / h \nu$.

When higher vibrational levels of the reactants are also involved, e.g., at finite temperatures, the expression for $k$ in Eqn. 13 contains Boltzmannweighted Franck-Condon factors and can be written as ${ }^{\S}$ :

$k=\frac{2 \pi}{\hbar h \nu} H_{A B}^{2} \mathrm{e}^{v y-S \operatorname{coth} y} I_{v}(S \operatorname{cosech} y)$

where $y=h \nu / 2 R T, I_{v}$ is a modified Bessel function, and the remaining symbols have their previous meaning.

We consider next some properties of these equations. At sufficiently high temperatures Eqn. 72 reduces to Eqn. $14^{\dagger}$, while at sufficiently low temperatures it reduces to Eqn. 71. Further, the $k$ given by Eqn. 71 has a maximum as a function of $v$ (i.e., of $-\Delta G^{0^{\prime}} / h \nu$ ) which occurs when $v \approx S$ (cf. Ref. 51). This result is seen by introducing Stirling's formula for $\Gamma(v+1)$, namely $\sqrt{2 \pi v}(v / \mathrm{e})^{v}$, maximizing the expression in Eqn. 71 , and neglecting the variation in $\sqrt{v}$ relative to that in $(v / \mathrm{e})^{v}$. Thus, the maximum occurs at $\lambda \approx$ $-\Delta G^{0^{\circ}}$, just as it did for the classical expression, Eqn. 14. Further, it was seen earlier from Eqn. 35 that the activation energy for the classical expression is zero at $\Delta G^{0^{\prime}}=-\lambda$. This same choice for $\lambda$ also leads to a similarly low activation energy when Eqn. 71 is used for the value of $k$ at $0 \mathrm{~K}$ and Eqn. 14 for the values at high temperatures, or hence when Eqn. 72 is used over the entire temperature range.

We next apply Eqns. $71-72$ and 14 to the kinetic data given in subsection IVD for the photosynthetic barrierless regime. In that regime, as already noted, $-\Delta G^{0^{\prime}} \approx \lambda$. It will be assumed, for the present purposes, that there is an averaged vibrational frequency $\nu$ for the contributing modes of about $200 \mathrm{~cm}^{-1}$ (i.e., $h \nu$ is $2.5 \mathrm{~kJ} \cdot \mathrm{mol}^{-1}$ ). (Larger quantum-mechanical corrections occur if $\nu$ is larger than the frequency used here [131].) If the driving force $-\Delta G^{0^{\prime}}$ in the first step (or two steps)

$\S$ Cf. previous footnote.

† At high temperatures $S$ coth $y$ and $S$ cosech $y$ both become $2 \lambda R T /(h \nu)^{2}$. Use of the high temperature form [328] of $I_{v}(x)$, namely $(2 \pi x)^{-1 / 2} \exp \left(x-v^{2} / 2 x\right)$, where $x=$ $2 \lambda R T /(h \nu)^{2}$, then yields Eqn. 14 from Eqn. 72. for electronically excited $(\mathrm{BChl})_{2} \rightarrow \mathrm{BChl} \rightarrow \mathrm{BPh}$ is about $0.25 \mathrm{eV}$ total, then the value per step is about $0.13 \mathrm{eV}$ or approx. $12.5 \mathrm{~kJ} \cdot \mathrm{mol}^{-1}$, and so, within the framework of the above analysis, a typical value of $\lambda$ for these reactants is expected to be in the neighborhood of $12.5 \mathrm{~kJ} \cdot \mathrm{mol}^{-1}$ if the reaction is to be barrierless. We consider several value of $\lambda$ below in the range $5-38 \mathrm{~kJ} \cdot \mathrm{mol}^{-1}$, together with the above values for $h \nu$ and $-\Delta G^{0}$.

We first compare at $25^{\circ} \mathrm{C}$ the classical Eqn. 14 with the quantum Eqn. 72 by comparing the factor multiplying $2 \pi H_{\mathrm{AB}}^{2} / h h \nu$ in those equations. (In Eqn. 14 this factor is $h \nu / \sqrt{4 \pi \lambda R T}$-times the exponential.) For the above factor such a comparison at $25^{\circ} \mathrm{C}$ yields, respectively, 0.063 and 0.063 $\left(\lambda=5.0 \mathrm{~kJ} \cdot \mathrm{mol}^{-1}\right), 0.13$ and $0.12(\lambda=8.4 \mathrm{~kJ}$. $\left.\mathrm{mol}^{-1}\right), 0.10$ and $0.10\left(\lambda=17 \mathrm{~kJ} \cdot \mathrm{mol}^{-1}\right)$, and 0.048 and $0.050\left(\lambda=25 \mathrm{~kJ} \cdot \mathrm{mol}^{-1}\right)$. Thus, the classical Eqn. 14 and the quantum Eqn. 72 agree closely when $h v / 2 R T$ is not large. (Here, it is 0.5 at $25^{\circ} \mathrm{C}$.) Analytically, Eqn. 14 is the 'high-temperature' limit of Eqn. 72, as already noted.

The temperature dependence of $k$ is seen by comparing the above results at $25^{\circ} \mathrm{C}$ with those obtained at $0 \mathrm{~K}$ using Eqn. 71: at $0 \mathrm{~K}$ the above factors are found to be $0.036,0.12,0.14$ and 0.038 for $\lambda=5.0,8.4,17$ and $25 \mathrm{~kJ} \cdot \mathrm{mol}^{-1}$, respectively. Thus, the results for $\lambda \leq 25 \mathrm{~kJ} \cdot \mathrm{mol}^{-1}$ show negligible temperature dependence for the assumed value of $-\Delta G^{0^{\prime}}$. At $\lambda=38 \mathrm{~kJ} \cdot \mathrm{mol}^{-1}$ there is, however, a significant temperature dependence: the factor mentioned above calculated from Eqn. 14 is 0.014 at $25^{\circ} \mathrm{C}$ and calculated from Eqn. 71 is 0.0019 at $0 \mathrm{~K}$. Larger $\lambda$ 's will show an even larger temperature dependence.

We consider next the absolute value of $k$. Since Eqns. 14 and 72 are seen above to give similar values for $k$ at room temperature in the general neighborhood of $\lambda=-\Delta G^{0^{\prime}}$ we shall simply use the classical equations $31-33 b$ for the present calculation of $k$. When $\Delta G^{0^{\prime}} \approx-\lambda, d \cong 0.1 \mathrm{~nm}$ and $\beta \approx 12 \mathrm{~nm}^{-1}$, Eqns. $31-33 \mathrm{~b}$ yield a value of $3 \cdot 10^{12} \mathrm{~s}^{-1}$, or a lifetime of $0.3 \mathrm{ps}$ for the reaction, while for $\lambda=5.0 \mathrm{~kJ} \cdot \mathrm{mol}^{-1}, k=1 \cdot 10^{12} \mathrm{~s}^{-1}$, which correspond to a lifetime of 1 ps. A $\lambda$ larger than $25 \mathrm{~kJ} \cdot \mathrm{mol}^{-1}$ will yield, for the given $\Delta G^{0^{\prime}}$, a smaller $k$ but will also give a significant temperature coefficient. The experimental value for the overall transfer is, as noted in the text, 4 to 5 ps. 
Thus, perhaps the assumed $1 \cdot 10^{13} \mathrm{~s}^{-1}$ in Eqn. $33 \mathrm{~b}$ is too high a value for the orientational configuration of the molecules involved, or perhaps there are high frequency modes (in addition to the others) which contribute a Franck-Condon factor, such as the one in Eqn. 71, that is temperature-independent over the entire investigated temperature range, $5-300 \mathrm{~K}$. The latter factor makes the rate lower by effectively contributing a temperature-independent factor to the rate constant expression.

We turn next to the $k$ for the $\mathrm{BPh}^{-}+\mathrm{Q}$ reaction. With a driving force in the vicinity of 0.5 to $0.6 \mathrm{eV}$ (subsection IVD) and a $\lambda$ of about this value (subsection IVD), the reaction is again barrierless. The values obtained from Eqns. 14 and 71 , or 72 may again be compared: with $\Delta G^{0^{\prime}} \cong$ $-\lambda=-0.5 \mathrm{eV}$, and $h \nu \cong 200 \mathrm{~cm}^{-1}$ the factor multiplying $2 \pi H_{\mathrm{AB}}^{2} / \hbar h \nu$ in Eqn. 71 is 0.09 , and in Eqn. 14 it is $h \nu /(4 \pi \lambda R T)^{1 / 2}$, namely, 0.06. Thus, once again the temperature coefficient is minor, as expected. Use of Eqns. $31-33 \mathrm{~b}$ with $d \approx 1.0 \mathrm{~nm}$ and $\beta \cong 12 \mathrm{~nm}^{-1}$ (subsection VD), yields the value of $5 \cdot 10^{9} \mathrm{~s}^{-1}$ in the text, corresponding to a lifetime of 200 ps.

Various authors have treated the rate constants in the photosynthetic system and used a wide variety of vibration frequencies and models (see for example, Refs. 306, 329 and 330 and see also Kirmaier, C., Holten, D. and Parson, W.W., unpublished results). The negative temperature effect for the $\mathrm{BPh}^{-}+\mathrm{Q}$ reaction of a factor of two in $k$ has been interpreted by the latter authors in terms of differences of vibration frequencies of reactants and products. However, in the present calculations, factors of two are not the focus of attention and possible effects of differences in vibration frequencies of reactants and products have been omitted.

\section{Acknowledgements}

We wish to acknowledge helpful discussions with Drs. J. Fajer, H.B. Gray, G. McLendon, T.L. Netzel, I. Pecht, A.G. Sykes and J.K. Yandell. We are also grateful to Dr. D. Holten for making kinetic data available prior to publication and Drs. P.C. Weber and F.R. Salemme for modeling the docking of two cytochrome $c$ molecules and for providing Fig. 9. Research at Brookhaven $\mathrm{Na}$ - tional Laboratory (N.S.) was carried out under contract DE-AC02-76CH00016 with the U.S. Department of Energy and supported by its Division of Chemical Sciences, Office of Basic Energy Sciences, while that at Caltech (R.A.M.) was supported by the National Science Foundation and the Office of Naval Research (contribution No. 7095 from Caltech).

\section{References}

1 Marcus, R.A. (1956) J. Chem. Phys. 24, 966-978

2 Marcus, R.A. (1960) Disc. Faraday Soc. 29, 21-31

3 Marcus, R.A. (1965) J. Chem. Phys. 43, 679-701

4 Marcus, R.A. (1968) J. Phys. Chem. 72, 891-899

5 Kresge, A.J. (1975) Acc. Chem. Res. 8, 354-360

6 Albery, W.J. (1980) Annu. Rev. Phys. Chem. 31, 227-263

7 Albery, W.J. and Kreevoy, M.M. (1978) Adv. Phys. Org. Chem. 16, 87-157

8 Donnella, J. and Murdoch, J.R. (1984) J. Am. Chem. Soc. $106,4724-4735$

9 Lewis, E.S. and Hu, D.D. (1984) J. Am. Chem. Soc. 106 , 3292-3296

10 Roberts, R.M.G., Ostovic, D. and Kreevoy, M.M. (1982) Faraday Disc. Chem. Soc. 74, 257-265

11 Kreevoy, M.M. and Lee, I.-S.H. (1984) J. Am. Chem. Soc. $106,2550-2553$

12 Magnoli, D.E., Murdoch, J.R. (1981) J. Am. Chem. Soc. $103,7465-7469$

13 Pellerite, M.J. and Brauman, J.I. (1980) J. Am. Chem. Soc. 102, 5993-5999

14 Wolfe, S., Mitchell, D.J., and Schlegel, H.B. (1981) J. Am. Chem. Soc. 103, 7694-7696

15 Chen, M.Y. and Murdoch, J.R. (1984) J. Am. Chem. Soc. $106,4735-4743$

16 Murdoch, J.R. (1982) Faraday Disc. Chem. Soc. 74, 297-306

17 Cannon, R.D. (1980) Electron Transfer Reactions, Butterworths, London

18 Reynolds, W.L. and Lumry, R.W. (1966) Mechanisms of Electron Transfer, Ronald Press, New York

19 DeVault, D. (1980) Q. Rev. Biophys. 13, 387-564

20 DeVault, D. (1984) Quantum-Mechanical Tunnelling in Biological Systems, 2nd Edn., Cambridge University Press, New York

21 Sutin, N. (1983) Progr. Inorg. Chem. 30, 441-498

22 Sutin, N. (1973) in Inorganic Biochemistry (Eichhorn, G.L., ed.) Vol. 2, pp. 611-653, Elsevier, New York

23 Newton, M.D. and Sutin, N. (1984) Annu. Rev. Phys. Chem. 35, 437-480

24 Marcus, R.A. (1979) Life Sci. Res. Rep. 12, 15-43

25 Marcus, R.A. (1964) Annu. Rev. Phys. Chem. 15, 155-196

26 Marcus, R.A. (1957) J. Chem. Phys. 26, 867-871

27 Marcus, R.A. (1982) Faraday Disc. Chem. Soc. 74, 7-15

28 Larsson, S. (1983) J. Chem. Soc. Faraday Trans. 2, 79, 1375-1388

29 Marcus, R.A. (1957) ONR Tech. Rept. No. 12, Project NR-051-331; reproduced (1977) in Special Topics in Elec- 
trochemistry (Rock, P.A. ed.), pp. 180-209, Elsevier, New York

30 Marcus, R.A. (1959) Can. J. Chem. 37, 155-163

31 Marcus, R.A. (1963) J. Phys. Chem. 67, 853-857, 2889

32 Landau, L.D. and Lifshitz, E.M. (1965) Quantum Mechanics, 2nd Edn., pp. 177-180, Pergamon Press, New York

33 Marcus, R.A. (1965) J. Chem. Phys. 43, 2654-2657

34 Marcus, R.A. (1970) J. Chem. Phys. 52, 2803-2804

35 Wallace, W.L. and Bard, A.J. (1979) J. Phys. Chem. 83, 1350-1357

36 Miller, J.R., Calcaterra, L.T., and Closs, G.L. (1984) J. Am. Chem. Soc. 106, 3047-3049

37 Miller, J.R., Beitz, J.V. and Huddleston, R.K. (1984) J. Am. Chem. Soc. 106, 5057-5068

38 Wasielewski, M.R., Niemczyk, M.P., Svec, W.A., Pewitt, E.B. (1985) J. Am. Chem. Soc. 107, 1080-1082

39 Allen, A.O. Gangwer, T.E. and Holroyd, R.A. (1975) J. Phys. Chem. 79, 25-31

40 Hush, N.S. (1961) Trans. Faraday Soc. 57, 557-580

41 George, P. and Griffith, J.S. (1959) in The Enzymes (Boyer,P.D., Lardy, H. and Myrbäck, K., eds.), 2nd Edn., Vol. 1, pp. 347-389, Academic Press, New York

42 Orgel, L.E. (1956) Inst. Intern. Chim. Solvay Conseil Chim. Brussels 10, 289-338

43 Kakitani, T. and Mataga, N. (1985) J. Phys. Chem. 89, 8-10

44 Sutin, N. (1966) Ann. Rev. Phys. Chem. 17, 119-172

45 Levich, V.G. and Dogonadze, R.R.(1959) Dokl. Acad. Nauk SSSR 124, 123-126 (Proc. Acad. Sci. Phys. Chem. Sect. 124, 9-13)

46 Levich, V.G. (1966) Adv. Electrochem. Electrochem. Eng. 4, 249-371

47 Dogonadze, R.R., Kuznetsov, A.M. and Vorotyntsev, M.A. (1972) Phys. Stat. Sol. B 54, 125-134 and 425-433

48 Van Duyne, R.P. and Fischer, S.F. (1974) Chem. Phys. 5, 183-197

49 Kestner, N.R., Logan, J. and Jortner, J. (1974) J. Phys. Chem. 78, 2148-2166

50 Ulstrup, J. and Jortner, J. (1975) J. Phys. Chem. 63, 4358-4368

51 Jortner, J. (1976) J. Chem. Phys. 64, 4860-4867

52 Efrima, S. and Bixon, M. (1976) Chem. Phys. 13, 417-460

53 Siders, P. and Marcus, R.A. (1981) J. Am. Chem. Soc. 103 , 741-747

54 Schmidt, P.P. (1978) Electrochemistry Spec. Period. Rep. 6, 128-241

55 Marcus, R.A. and Siders, P. (1982) J. Phys. Chem. 86, $622-630$

56 Warshel, A. (1982) J. Phys. Chem. 86, 2218-2224

57 Ulstrup, J. (1979) Charge Transfer Processes in Condensed Media, Lecture Notes in Chemistry, No. 10, SpringerVerlag, New York

58 Marcus, R.A. (1984) J. Chem. Phys. 81, 4494-4500

59 Marcus, R.A. (1973) Faraday Disc. Chem. Soc. 55, 34-44

60 Marcus, R.A. and Sutin, N. (1975) Inorg. Chem. 14, 213-216

61 Siders, P. and Marcus, R.A. (1981) J. Am. Chem. Soc. 103, $748-752$
62 Buhks, E., Bixon, M., Jortner, J. and Navon, G. (1981) J. Phys. Chem. 85, 3759-3762

63 Hopfield, J.J. (1974) Proc. Natl. Acad. Sci. USA 71, 3640-3644

64 Hopfield, J.J. (1977) in Electrical Phenomena at the Biological Membrane Level (Roux, E., ed.), pp. 471-492, Elsevier, New York

65 Marcus, R.A. (1982) in Oxidases and Related Redox Systems (King, T.E., Mason, H.S. and Morrison, M., eds.), pp. 3-19, Pergamon Press, Oxford

66 Holstein, T. (1979) in Tunneling in Biological Systems (Chance, B., DeVault, D.C., Frauenfelder, H., Marcus, R.A., Schrieffer, J.B. and Sutin, N., eds.), pp. 129-136, Academic Press, New York

67 Holstein, T. (1978) Philos. Mag. B, 37, 49-62

68 Scher, H. and Holstein, T. (1981) Philos Mag. B, 44, 343-356

69 Brunschwig, B.S., Logan, J., Newton, M.D. and Sutin, N. (1980) J. Am. Chem. Soc. 102, 5798-5809

70 Larsson, S. (1981) J. Am. Chem. Soc. 103, 4031-4040

71 Beratan, D.N. and Hopfield, J.J. (1984) J. Chem. Soc. 106, 1584-1594

72 Siders, P., Cave, R.J. and Marcus, R.A. (1984) J. Chem. Phys. 81, 5613-5624

73 Reference deleted

74 Reference deleted

75 Chang, R. and Weismann, S.I. (1967) J. Am. Chem. Soc. 89,5968

76 Tembe, B.L., Friedman, H.L. and Newton, M.D. (1982) J. Chem. Phys. 76, 1490-1507

77 Newton, M.D. (1982) Am. Chem. Soc. Symp. Ser. 198, 255-279

78 Brunschwig, B.S., Creutz, C., Macartney, D.H., Sham, T.K. and Sutin, N. (1982) Faraday Disc. Chem. Soc. 74, 113-127

79 Chou, M., Creutz; C. and Sutin, N. (1977) J. Am. Chem. Soc. $99,5615-5623$

80 Weaver, M.J. and Yee, E.L. (1980) Inorg. Chem. 19, 1936-1945

81 Pennington, D.E. (1978) in Coordination Chemistry (Martell, A.E., ed.) Vol. 2, Am. Chem. Soc. Monograph No. 174 , pp. $476-590$

82 Frese, Jr., K.W. (1981) J. Phys. Chem. 85, 3911-3916

83 Hupp, J.T. and Weaver, M.J. (1983) Inorg. Chem. 22, 2557-2564

84 Meyer, T.J. and Taube, H. (1968) Inorg. Chem. 7, 2369-2379

85 Campion, R.J., Purdie, N. and Sutin, N. (1964) Inorg. Chem. 3, 1091-1094

86 Thomas, L. and Hicks, K.W. (1974) Inorg. Chem. 13, 749-752

87 Rawoof, M.A. and Sutter, J.R. (1967) J. Phys. Chem. 71, 2767-2771

88 Böttcher, W., Brown, G.M. and Sutin, N. (1979) Inorg. Chem. 18, 1447-1451

89 Lin, C.-T., Böttcher, W., Chou, M., Creutz, C. and Sutin, N. (1976) J. Am. Chem. Soc. 98, 6536-6544

90 Endicott, J.F., Durham, B. and Kumar, K. (1982) Inorg. Chem. 21, 2437-2444 
91 Silverman, J. and Dodson, R.W. (1952) J. Phys. Chem. 56, 846-851

92 Bernhard, P., Bürgi, H.B., Hauser, J., Lehmann, H. and Ludi, A. (1982) Inorg. Chem. 21, 3936-3941

93 Young, R.C., Keene, F.R. and Meyer, T.J. (1977) J. Am. Chem. Soc. 99, 2468-2473

94 Hammershoi, A., Geselowitz, D. and Taube, H. (1984) Inorg. Chem. 23, 979-982

95 Neumann, H.M., quoted in: Farina, R. and Wilkins, R.G. (1968) Inorg. Chem. 7, 514-518

96 Brown, G.M. and Sutin, N. (1979) J. Am. Chem. Soc. 101, 883-892

97 Li, T.T.-T. and Brubaker, C.H., Jr. (1981) J. Organomet. Chem. 216, 223-234

98 Chan, M.-S. and Wahl, A.C. (1982) J. Phys. Chem. 86, 126-130

99 Brandon, J.R. and Dorfman, L.M. (1970) J. Chem. Phys. $53,3849-3856$

100 Yang, E.S., Chan, M.-S. and Wahl, A.C. (1980) J. Phys. Chem. 84, 3094-3099

101 Creutz, C. and Sutin, N. (1985) in Inorganic Reactions and Methods (Zuckerman, J.J., ed.), Springer-Verlag, in the press

102 Ford-Smith, M.H. and Sutin, N. (1961) J. Am. Chem. Soc. $83,1830-1834$

103 Dulz, G. and Sutin, N. (1963) Inorg. Chem. 2, 917-921

104 Brunschwig, B.S. and Sutin, N. (1979) Inorg. Chem. 18, 1731-1736

105 Brunschwig, B.S. and Sutin, N. (1978) J. Am. Chem. Soc. $100,7568-7577$

106 Pelizzetti, E., Mentasti, E. and Pramauro, E. (1978) Inorg. Chem. 17, 1181-1186

107 Macartney, D.H. and Sutin, N. (1983) Inorg. Chim. Acta, $74,221-228$

108 Pelizzetti, E. and Mentasti, E. (1979) Inorg. Chem. 18, 583-588

109 Fukuzumi, S., Wong, C.L., and Kochi, J.K. (1980) J. Am. Chem. Soc. 102, 2928-2939

110 Bock, C.P., Connor, J.A., Gutierrez, A.R., Meyer, T.J., Whitten, D.G., Sullivan, B.P. and Nagle, J.K. (1979) J. Am. Chem. Soc. 101, 4815-4824

111 Meyer, T.E., Przysiecki, C.T., Watkins, J.A., Bhattacharyya, A., Simondsen, R.P., Cusanovich, M.A. and Tollin, G. (1983) Proc. Natl. Acad. Sci. USA 80, 6740-6744

112 Hush, N.S. (1967) Prog. Inorg. Chem. 8, 391-444

113 Creutz, C. (1983) Prog. Inorg. Chem. 30, 1-73

114 Marcus, R.A. (1965) J. Chem. Phys. 43, 1261-1274

115 Marcus, R.A. and Sutin, N. (1985) Comments Inorg. Chem. 4 , in the press

116 Anderson, P.W. (1965) in Magnetism (Rado, G.T. and Suhl, H., eds.), Vol. 1, pp. 25-81, Academic Press, New York

117 Okamura, M.Y., Fredkin, D.R., Isaacson, R.A. and Feher, G. (1979) in Tunneling in Biological Systems (Chance, B., DeVault, D.C., Frauenfelder, H., Marcus, R.A., Schrieffer, J.B. and Sutin, N., eds.), pp. 729-743, Academic Press, New York

118 Creutz, C. (1978) Inorg. Chem. 17, 3723-3725
119 Sutton, J.E., Sutton, P.M. and Taube, H. (1979) Inorg. Chem. 18, 1017-1021

120 Rieder, K. and Taube, H. (1977) J. Am. Chem. Soc. 99, 7891-7894

121 Creutz, C. and Taube, H. (1973) J. Am. Chem. Soc. 95, 1086-1094

122 Fürholz, U., Burgi, H.-B., Wagner, F.E., Stebler, A., Ammeter, J.H., Krausz, E., Clark, R.J.H., Stead, M.J. and Ludi, A. (1984) J. Am. Chem. Soc. 106, 121-123

123 Root, L.J. and Ondrechen, M.J. (1982) Chem. Phys. Lett. 93, 421-424

124 Ondrechen, M.J., Ellis, D.L. and Ratner, M.A. (1984) Chem. Phys. Lett. 109, 50-55

125 Ko, J. and Ondrechen, M.J. (1984) Chem. Phys. Lett. 112, 507-512

126 Curtis, J.C. and Meyer, T.J. (1982) Inorg. Chem. 21, 1562-1571

127 Stein, C.A. and Taube, H. (1981) J. Am. Chem. Soc. 103, 693-695

128 Stein, C.A., Lewis, N.A. and Seitz, G. (1982) J. Am. Chem. Soc. 104, 2596-2599

129 Redi, M. and Hopfield, J.J. (1980) J. Chem. Phys. 72, $6651-6660$

130 Delahay, P. and Dziedzic, A. (1984) J. Chem. Phys. 80, 5381-5387

131 Delahay, P. and Dziedzic, A. (1984) J. Chem. Phys. 80, 5793-5798

132 Saji, T., Maruyama, Y., and Aoyagui, S. (1978) J. Électroanal. Chem. 86, 219-222

133 Weaver, M.J. (1980) J. Phys. Chem. 84, 568-576

134 Weaver, M.J. and Hupp, J.T. (1982) Am. Chem. Soc. Symp. Series 198, 181-208

135 Saveant, J.-M. and Tessier, D. (1982) Faraday Disc. Chem. Soc. 74, 57-72

136 Rackovsky, S. and Goldstein, D.A. (1984) Proc. Natl. Acad. Sci. USA 81, 5901-5905

137 Churg, A.K., Weiss, R.M., Warshel, A. and Takano, T. (1983) J. Phys. Chem. 87, 1683-1694

138 Alexandrov, I.V., Khairutdinov, R.F. and Zamaraev, K.I. (1978) Chem. Phys. 32, 123-141

139 Miller, J.R. (1975) Science 189, 221-222

140 Reference deleted

141 Miller, J.R. and Beitz, J.V. (1981) J. Chem. Phys. 74, 6746-6756

142 Calcaterra, L.T., Closs, G.L. and Miller, J.R. (1983) J. Am. Chem. Soc. 105, 670-671

143 Mann, B. and Kuhn, H. (1971) J. Appl. Phys. 42, 4398-4405

144 Polymeropoulos, E.E. (1977) J. Appl. Phys. 48, 2404-2407

145 Sugi, M., Fukui, T. and Iizima, S. (1975) Appl. Phys. Lett. 27, 559-561

146 Sugi, M., Nembach, K., Möbius, D. and Kuhn, H. (1974) Sol. Stat. Comm. 15, 1867-1870

147 Kuhn, H. (1979) J. Photochem. 10, 111-132

148 Killesreiter, H. and Baessler, H. (1971) Chem. Phys. Lett. $11,411-414$

149 Killesreiter, H. and Baessler, H. (1972) Phys. Stat. Sol. B $51,657-668$ 
150 Lindsey, J.S., Mauzerall, D.C., and Linschitz, H. (1983) J. Am. Chem. Soc. 105, 6528-6529

151 Kostić, N.M., Margalit, R., Che, C.-M. and Gray, H.B. (1983) J. Am. Chem. Soc. 105, 7765-7767

152 McCourty, J.L., Blough, N.V. and Hoffman, B.M. (1983) J. Am. Chem. Soc. 105, 4470-4472

153 Peterson-Kennedy, S.E., McGourty, J.L. and Hoffman, B.M. (1984) J. Am. Chem. Soc. 106, 5010-5012

154 McLendon, G.L., Winkler, J.R., Nocera, D.G., Mauk, M.R., Mauk, A.G. and Gray, H.B. (1985) J. Am. Chem. Soc., 107, 739-740

155 Nocera, D.G., Winkler, J.R., Yokom, K.M., Bordignon, E. and Gray, H.B. (1984) J. Am. Chem. Soc. 106, 5145-5150

156 Isied, S.S., Kuehn, C. and Worosila, G. (1984) J. Am. Chem. Soc. 106, 1722-1726

157 Isied, S.S. (1984) Progr. Inorg. Chem. 32, 443-517

158 Brunschwig, B.S., Ehrenson, S. and Sutin, N. (1984) J. Am. Chem. Soc. 106, 6858-6859

159 Wherland, S. and Gray, H.B. (1977) in Biological Aspects of Inorganic Chemistry, (Addison, A.W., Cullen, W.R., Dolphin, D. and James, B.R., eds.), pp. 289-368, Wiley, New York

160 Margalit, R. and Schejter, A. (1973) Eur. J. Biochem. 32, 492-499

161 Koppenol, W.H., Vroonland, C.A.J. and Braams, R. (1978) Biochim. Biophys. Acta 503, 499-508

162 Morton, R.A., Overnell, J. and Harbury, H.A. (1970) J. Biol. Chem. 245, 4653-4657

163 Goldkorn, T. and Schejter, A. (1979) J. Biol. Chem. 254, $12562-12566$

164 McArdle, J.V., Coyle, C.L., Gray, H.B., Yoneda, G.S. and Holwerda, R.A. (1977) J. Am. Chem. Soc. 99, 2483-2489

165 Rawlings, J., Wherland, S. and Gray, H.B. (1977) J. Am. Chem. Soc. 99, 1968-1971

166 Segal, M.G. and Sykes, A.G. (1978) J. Am. Chem. Soc. $100,4585-4592$

167 Sailasuta, N., Anson, F.C. and Gray, H.B. (1979) J. Am. Chem. Soc. 101, 455-458

168 Yee, E.L. and Weaver, M.J. (1980) Inorg. Chem. 19, 1077-1079

169 Feinberg, B.A., Ryan, M.D. and Wei, J.-F. (1977) Biochem. Biophys. Res. Commun. 79, 769-775

170 Feinberg, B.A. and Ryan, M.D. (1981) in Topics in Bioelectrochemistry and Bioenergetics (Milazzo, G., ed.), Vol. 4, 225-270, Wiley and Sons, New York

171 Rosenberg, R.C., Wherland, S., Holwerda, R.A. and Gray, H.B. (1976) J. Am. Chem. Soc. 98, 6364-6369

172 Koppenol, W.H. (1980) Biophys. J. 29, 493-507

173 Koppenol, W.H. and Margoliash, E. (1982) J. Biol. Chem. 257, 4426-4437

174 Van Leeuwen, J.W., Mofers, F.J.M. and Veerman, E.C.I. (1981) Biochim. Biophys. Acta, 635, 431-439

175 Van Leeuwen, J.W. (1983) Biochim. Biophys. Acta 743, 408-421

176 Bronsted, J.N. (1922) J. Am. Chem. Soc. 44, 877-898

177 Meyer, T.E., Watkins, J.A., Przysiecki, C.T., Tollin, G. and Cusanovich, M.A. (1984) Biochem., 23, 4761-4767

178 Chapman, S.K., Sinclair-Day, J.D., Sykes, A.G., Tam, S.C. and Williams, R.J.P. (1983) J. Chem. Soc., Chem. Commun., 1152-1154

179 Beoku-Betts, D. and Sykes, A.G. (1985) Inorg. Chem. 24, 1142-1147

180 Matthew, J.B., Weber, P.C., Salemme, F.R. and Richards, F.M. (1983) Nature 301, 169-171

181 Dickerson, R.E. and Timkovich, R., (1975) in The Enzymes (Boyer, P.D., ed.) 3rd Edn., Vol. XIA, pp. 397-547, Academic Press, New York

182 Takano, T. and Dickerson, R.E. (1981) J. Mol. Biol. 153, 95-115

183 Moore, G.R., Huang, Z.-X., Eley, C.G.S., Barker, H.A., Williams, G., Robinson, M.N. and Williams, R.J.P. (1982) Faraday Disc. Chem. Soc. 74, 311-329

184 Brown, G.M., Krentzien, H.J., Abe, M. and Taube, H. (1979) Inorg. Chem. 18, 3374-3379

185 Sutin, N. (1977) Am. Chem. Soc. Adv. Chem. Ser. 162, 156-172

186 Wilkins, R.G. and Yelin, R.E. (1968) Inorg. Chem. 7, 2667-2669

187 Campion, R.J., Deck, C.F., King, P. and Wahl, A.C. (1967) Inorg. Chem. 6, 672-681

188 McArdle, J.V., Gray, H.B., Creutz, C. and Sutin, N. (1974) J. Am. Chem. Soc. 96, 5737-5741

189 Ewall, R.X. and Bennett, L.E. (1974) J. Am. Chem. Soc. 96, 940-942

190 Cummins, D. and Gray, H.B. (1977) J. Am. Chem. Soc. $99,5158-5167$

191 Creutz, C. and Sutin, N. (1974) J. Biol. Chem. 249, 6788-6795

192 Hodges, H.L., Holwerda, R.A. and Gray, H.B. (1974) J. Am. Chem. Soc. 96, 3132-3137

193 Gupta, R.K. (1973) Biochim. Biophys. Acta 292, 291-295

194 Holwerda, R.A., Knaff, D.B., Gray, H.B., Clemmer, J.D., Crowley, R., Smith, J.M. and Mauk, A.G. (1980) J. Am. Chem. Soc. 102, 1142-1146

195 Butler, J., Davies, D.M., Sykes, A.G., Koppenol, W.H., Osheroff, N. and Margoliash, E. (1981) J. Am. Chem. Soc. $103,469-471$

196 Ohno, N. and Cusanovich, M.A. (1981) Biophys. J. 36, 589-605

197 Butler, J., Davies, D.M. and Sykes, A.G. (1981) J. Inorg. Biochem. 15, 41-53

198 Chapman, S.K., Davies, D.M., Watson, A.D. and Sykes, A.G. (1983) Am. Chem. Soc. Symp. Series 211, 177-194

199 Potasek, M.J. and Hopfield, J.J. (1977) Proc. Natl. Acad. USA 74, 229-233

200 Dickerson, R.E. (1979) in Tunneling in Biological Systems (Chance, B., DeVault, D.C., Frauenfelder, H., Marcus, R.A., Schrieffer, J.B. and Sutin, N. eds.), p. 545, Academic Press, New York

201 Austin, R.H. and Hopfield, J.J., cited in: Redi, M. and Hopfield, J.J. (1980) J. Chem. Phys. 72, 6651-6660

202 Harbury, H.A. and Marks, R.H.L. (1973) in Inorganic Biochemistry, (Eichhorn, G.L., ed.), Vol. 2, pp. 902-954, Elsevier, New York

203 Reference deleted

204 Schomacker, K.T., Bangcharoenpaurpong, O. and Champion, P.M. (1984) J. Chem. Phys. 80, 4701-4717 
205 Dixon, D.W., Barbush, M. and Shiragi, A. (1984) J. Am. Chem. Soc. 106, 4638-4639

206 Keller, R.M., Wüthrich, K. and Pecht, I. (1976) FEBS Lett. 70, 180-184

207 Yokom, K.M., Ph.D. Thesis, California Institute of Technology, Pasadena, CA., 1982

208 Margalit, R., Kostić, N.M., Che, C.-M., Blair, D.F., Chiang, H.-J., Pecht, I., Shelton, J.R., Schroeder, W.A. and Gray, H.B. (1984) Proc. Natl. Acad. Sci. USA 81, 6554-6558

209 Winkler, J.R., Nocera, D.G., Yokom, K.M., Bordignon, E. and Gray, H.B. (1982) J. Am. Chem. Soc. 104, 5798-5800

210 Cho, K.C., Che, C.M., Cheng, F.C. and Choy, C.L. (1984) J. Am. Chem. Soc., 106, 6843-6844

211 Dutton, P.L., Leigh, J.S., Prince, R.C. and Tiede, D.M. (1979) in Tunneling in Biological Systems (Chance, B., DeVault, D.C., Frauenfelder, H., Marcus, R.A., Schrieffer, J.B. and Sutin, N. eds.), pp. 319-352, Academic Press, New York

212 Blankenship, D.E. and Parson, W.W. (1979) in Photosynthesis in Relation to Model Systems (Barber, J., ed.), pp. 71-114, Elsevier, New York

213 DeVault, D. and Chance, B. (1966) Biophys. J. 6, 825-847

214 Tiede, D.M., Leigh, J.S. and Dutton, P.L. (1978) Biochim. Biophys. Acta 503, 524-544

215 Buhks, E., Bixon, M. and Jortner, J. (1981) Chem. Phys. $55,41-48$

216 Jortner, J. (1980) Biochim. Biophys. Acta 594, 193-230

217 Deisenhofer, J., Epp, O., Miki, K., Huber, R. and Michel, H. (1984) J. Mol. Biol. 180, 385-398

218 Feher, G., Hoff, A.J., Isaacson, R.A. and Ackerson, L.C. (1975) Ann. N.Y. Acad. Sci. 244, 239-259

219 Norris, J.R., Scheer, H. and Katz, J.J. (1975) Ann. N.Y. Acad. Sci. 244, 261-280

220 Davis, M.S., Forman, A. Hanson, L.K., Thornber, J.P. and Fajer, J. (1979) J. Phys. Chem. 83, 3325-3332

221 Haberkorn, H., Michel-Beyerle, M.E. and Marcus, R.A. (1979) Proc. Natl. Acad. Sci. USA 76, 4185-4188

222 Thornber, J.P. and Olson, J.M. (1971) Photochem. Photobiol. 14, 329-341

223 Prince, R.C., Leigh, J.S. and Dutton, P.L. (1976) Biochim. Biophys. Acta 440, 622-636

224 Holten, D., Windsor, M.W., Parson, W.W. and Thornber, J.P. (1978) Biochim. Biophys. Acta 501, 112-126

225 Kihara, T. and McCray, J.A. (1973) Biochim. Biophys. Acta 292, 297-309

226 Netzel, T.L., Bergkamp, M.A. and Chang, C.-K. (1982) J. Am. Chem. Soc. 104, 1952-1957

227 Fujita, I., Fajer, J., Chang, C.-K., Wang, C.-B., Bergkamp, M.A. and Netzel, T.L. (1982) J. Phys. Chem. 86, 3754-3759

228 Netzel, T.L. (1982) in Biological Events Probed by Ultrafast Laser Spectroscopy (Alfano, R.R., ed.), pp. 79-117, Academic Press, New York

229 Mataga, N., Karen, A. and Okada, T. (1984) J. Am. Chem. Soc. 106, 2442-2443

230 Holten, D., Hoganson, C., Windsor, M.W., Schenck, C.C., Parson, W.W., Migus, A., Fork, R.L. and Shank, C.V. (1980) Biochim. Biophys. Acta 592, 461-472

231 Zinth, W., Franz, M.A., Nuss, M.C., Kaiser, W. and Michel, H. (1985) in Workshop on Antennas and Reaction
Centers of Photosynthetic Bacteria-Structure, Interactions and Dynamics (Michel-Beyerle, M.E., ed.), SpringerVerlag, Heidelberg, in the press

232 Shuvalov, V.A. and Klevanik, A.V. (1983) FEBS Lett. 160, 51-55

233 Closs, G.L. and Sitzmann, E.V. (1981) J. Am. Chem. Soc. 103, 3217-3219

234 Zankel, K.L., Reed, D.W. and Clayton, R.K. (1968) Proc. Natl. Acad. Sci. U.S.A. 61, 1243-1249

235 Dutton, P.l. (1971) Biochim. Biophys. Acta 226, 63-80

236 Woodbury, N.W.T. and Parson, W.W. (1984) Biochim. Biophys. Acta 767, 345-361

237 Klimov, V.V., Shuvalov, V.A., Krakhmaleva, I.N., Klevanik, A.V. and Krasnovsky, A.A. (1977) Biokhimia 42, 519-530

238 Pacham, N.K., Berriman, J.A. and Jackson, J.B. (1978) FEBS Lett. 89, 205-210

239 Reference deleted

240 Sutin, N. (1982) Accounts Chem. Res. 15, 275-282

241 Prince, R.C. and Dutton, P.L. (1976) Arch. Biochem. Biophys. 172, 329-334

242 Gunner, M.R., Liang, Y., Nagus, D.K., Hochstrasser, R.M. and Dutton, P.L. (1982) Biophys. J. 37, 226A

243 Pellin, M.J., Wraight, C.A. and Kaufman, K.J. (1978) Biophys. J. 24, 361-369

244 Wasielewski, M.R. and Niemczyk, M.P. 1(1984) J. Am. Chem. Soc. 106, 5043-5045

245 Chapman, S.K., Davies, D.M., Vuik, C.P.J. and Sykes, A.G. (1984) J. Am. Chem. Soc. 106, 2692-2696

246 Salemme, F.R. (1976) J. Molec. Biol. 102, 563-568

247 Salemme, F.R. (1977) Annu. Rev. Biochem. 46, 299-329

248 Dailey, H.A. and Strittmatter, P. (1979) J. Biol. Chem. 254, 5388-5396

$249 \mathrm{Ng}$, S., Smith, M.B., Smith, H.T. and Millet, F. (1977) Biochem. 16, 4975-4978

250 Strittmatter, P. (1964) in Rapid Mixing and Sampling Techniques in Biochemistry (Chance, B., Eisenhardt, R.H., Gibson, Q.H. and Lonberg-Holm, K.K., eds.), pp. 71-84, Academic Press, New York

251 Mauk, M.R., Reid, L.S. and Mauk, A.G. (1982) Biochem. 21, 1843-1846

252 Argos, P. and Mathews, F.S. (1975) J. Biol. Chem. 250, 747-751

253 Reid, L.S., Taniguchi, V.T., Gray, H.B. and Mauk, A.G. (1982) J. Am. Chem. Soc. 104, 7516-7519

254 Reference deleted

255 Simolo, K.P., McLendon, G.L., Mauk, M.R. and Mauk, A.G. (1984) J. Am. Chem. Soc. 106, 5012-5013

256 Malmström, B. (1979) Biochim. Biophys. Acta 549, 281-303

257 Brudvig, G.W., Blair, D.F. and Chan, S.I. (1984) J. Biol. Chem. 259, 11001-11009

258 Andreasson, L.E. (1975) Eur. J. Biochem. 53, 591-597

259 Wilson, M.T., Greenwood, C., Brunori, M. and Antonini, E. (1975) Biochem. J. 147, 145-153

260 Greenwood, C., Brittain, T., Wilson, M. and Brunori, M. (1976) Biochem. J. 157, 591-598

261 Yonetani, T. and Ray, G.S. (1965) J. Biol. Chem. 240, 3392-3398 
262 Dockter, M.E., Steinemann, A. and Schatz, G. (1978) J. Biol. Chem. 253, 311-317

263 Farver, O. and Pecht, I. (1984) in Copper Proteins and Copper Enzymes (Lontie, R., ed.), pp. 183-215, CRC Press, Boca Raton, FL

264 Colman, P.M., Freeman, H.C., Guss, J.M., Murata, M., Norris, V.A., Ramshaw, J.A.M. and Venkatappa, M.P. (1980) Nature 272, 319-324

265 Adman, E.T., Stenkamp, R.E., Sieker, L.C. and Jensen, L.H. (1978) Mol. Biol. 123, 35-47

266 Solomon, E.I., Hare, J.W., Dooley, D.M., Dawson, J.H., Stephens, P.J. and Gray, H.B. (1980) J. Am. Chem. Soc. 102, 168-178

267 Cookson, D.J., Hayes, M.T. and Wright, P.E. (1980) Biochem. Biophys. Acta 591, 162-176

268 Lappin, A.G., Segal, M.G., Weatherburn, D.C. and Sykes, A.G. (1979) J. Chem. Soc. Chem. Comm. 38-39

269 Handford, P.M., Hill, H.A.O., Lee, R.W.-K., Henderson, R.A. and Sykes, A.G. (1980) J. Inorg. Biochem. 13, 83-88

270 Wherland, S., Holwerda; R.A., Rosenberg, R.C. and Gray, H.B. (1975) J. Am. Chem. Soc. 97, 5260-5262

271 Chapman, S.K., Knox, C.V., Kathirgamathan, P. and Sykes, A.G. (1984) J. Chem. Soc. Dalton Trans. 2769-2773

272 Lappin, A.G. (1981) in Metal Ions in Biological Systems, (Sigel, H. ed.), Vol. 13, pp. 15-71, Marcel Dekker, New York

273 Augustin, M.A., Chapman, S.K., Davies, D.M., Watson, A.D. and Sykes, A.G. (1984) J. Inorg. Biochem. 20, 281-289

274 Sisley, M.J., Segal, M.G., Stanley, C.S., Adzamli, I.K. and Sykes, A.G. (1983) J. Am. Chem. Soc. 105, 222-228

275 Taniguchi, V.T., Sailasuta-Scott, N., Anson, F.C. and Gray, H.B. (1980) Pure and Appl. Chem. 52, 2275-2281

276 Canters, G.W., Hill, H.A.O., Kitchen, N.A. and Adman, E.T. (1984) J. Mag. Res. 57, 1-23

277 Beattie, J.K., Fensom, D.J., Freeman, H.C., Woodcock,E., Hill, H.A.O. and Stokes, A.M. (1975) Biochim. Biophys. Acta 405, 109-114

278 Dahlin, S., Reinhammar, B. and Wilson, M.T., (1984) Biochem. J. 218, 609-614

279 Farver, O. and Pecht, I. (1981) in Copper Proteins (Spiro, T.G., ed.), pp. 151-192, Wiley, New York

280 Goldberg, M. and Pecht, I. (1976) Biochem. 15, 4197-4208

281 Mauk, A.G., Scott, R.A. and Gray, H.B. (1980) J. Am. Chem. Soc. 102, 4360-4363

282 Brunswig, B.S., De Laire, P.J., English, A.M., Goldberg, M., Gray, H.B. and Sutin, N. (1985) Inorg. Chem. 24, in the press

283 Wilson, M.T., Silvestrini, M.C., Morpurgo, L. and Brunori, M. (1979) J. Inorg. Biochem. 11, 95-100

284 Wherland, S. and Pecht, I. (1978) Biochem. 17, 2585-2591

285 Norris, G.E., Anderson, B.F. and Baker, E.N. (1983) J. Mol. Biol. 165, 501-521

286 Moore, G.R. and Williams, R.J.P. (1976) Coord. Chem. Rev. 18, 125-197

287 Yonetani, T. and Ray, G.S. (1966) J. Biol. Chem. 241, 700-706

288 Kang, C.H., Ferguson-Miller, S. and Margoliash, E. (1978) J. Biol. Chem. 252, 919-926
289 Kang, C.H., Brautigan, D.L., Osheroff, N. and Margoliash, E. (1978) J. Biol. Chem. 253, 6502-6510

290 Purcell, W.L. and Erman, J.E. (1976) J. Am. Chem. Soc. 98, 7033-7037

291 Yandell, J.K. and Yonetani, T. (1983) Biochim. Biophys. Acta 748, 263-270

292 Yonetani, T. (1976) in The Enzymes (Boyer, P.D., ed.), Vol. 13, pp. 345-361, Academic Press, New York

293 Ho, P.S., Hoffman, B.M., Kang, C.H. and Margoliash, E. (1983) J. Biol. Chem. 258, 4356-4363

294 Poulos, T.L. and Kraut, J. (1980) J. Biol. Chem. 255, 8199-8205

295 Fujita, I., Hanson, L.K., Walker, F.A. and Fajer, J. (1983) J. Am. Chem. Soc. 105, 3296-3300

296 Poulos, T.L., Freer, S.T., Alden, R.A., Edwards, S.L., Skoglund, U., Takio, K., Eriksson, B., Xuong, N.-H., Yonetani, T. and Kraut, J. (1980) J. Biol. Chem. 255, 575-580

297 Poulos, T.L. and Kraut, J. (1980) J. Biol. Chem. 255, 10322-10330

298 Kraut, J. (1981) Biochem. Soc. Trans. 9, 197-202

299 Finzel, B.C., Poulos, T.L. and Kraut, J. (1984) J. Biol. Chem. 259, 13027-13036

300 Marcus, R.A. (1960) Disc. Faraday Soc. 29, 129-130

301 Noyes, R.M. (1961) Prog. Reaction Kinetics 1, 129-160

302 Debye, P. (1942) Trans. Amer. Electrochem. Soc. 82, 265-271

303 Sutin, N. and Brunschwig, B.S. (1982) Am. Chem. Soc. Symp. Ser. 198, 105.-125

304 Marcus, R.A. (1981) Int. J. Chem. Kinetics 13, 865-872

305 Dogonadze, R.R. (1971) in Reactions of Molecules at Electrodes (Hush, N.S., ed.), Wiley, New York, Ch. 3, Eqn. 201

306 Jortner, J. (1960) J. Am. Chem. Soc. 102, 6676-6686

307 Beratan, D.N. and Hopfield, J.J. (1984) J. Chem. Phys. 81, 5753-5759

308 Marcus, R.A. (1977) in Special Topics in Electrochemistry (Rock, P.A., ed.), pp. 161-179, Elsevier, New York

309 McConnell, H.M. (1961) J. Chem. Phys. 35, 508-515

310 Kuznetsov, A.M. and Ulstrup, J. (1981) J. Chem. Phys. 75, 2047-2055

311 Davydov, A.S. (1978) Phys. Stat. Sol. B. 90, 457-464

312 Larsson, S. (1984) J. Phys. Chem. 88, 1321-1323

313 Halpern, J. and Orgel, L.E. (1960) Disc. Faraday Soc. 29, 32-41

314 Kosower, E.M. and Huppert, D. (1983) Chem. Phys. Letter. $96,433-435$

315 Ovchinnikova, M.Ya. (1981) Teor. Eksp. Khim. 17, 651-654 (Theor. Exper. Chem. 17, 507-510)

316 Calef, D.F. and Wolynes, P.G. (1983) J. Phys. Chem. 87, 3387-3400

317 Reference deleted

318 Kakitani, T. and Mataga, N. (1985) Chem. Phys. 93, 381-397

319 Dietz, R. and Peover, M.E. (1968) Discussions Faraday Soc. 45, 154-166

320 Kojima, H. and Bard, A.J. (1975) J. Am. Chem. Soc. 97, $6317-6324$ 
321 Kojima, H. and Bard, A.J. (1975) J. Electroanal. Chem. 63, 117-129

322 Peover, M.E. and Powell, J.S. (1969) J. Electroanal. Chem. $20,427-433$

323 Huebert, B.J. and Smith, D.E. (1971) J. Electroanal. Chem. $31,333-348$

324 Garreau, D., Saveant, J.E. and Tessier, D. (1979) J. Phys. Chem. 83, 3003-3007

325 Fawcett, W.R. and Lasia, A. (1978) J. Phys. Chem. 82, 1114-1121

326 Rehm, D. and Weller, A. (1969) Ber. Bunsenges. Phys. Chem. 73, 834-839
327 Markham, J.J. (1959) Rev. Mod. Phys. 31, 956-989

328 Ballhausen, C. (1979) Molecular Electronic Structures of Transition Metal Complexes, pp. 112-115, McGraw-Hill, Maidenhead

329 Sarai, A. (1980) Biochim. Biophys. Acta 589, 71-83

330 Kakitani, T. and Kakitani, H. (1981) Biochim. Biophys. Acta $635,498-514$

331 Marcus, R.A. and Sutin, N. (1985) in Workshop on Antennas and Reaction Centers of Photosynthetic BacteriaStructure, Interactions and Dynamics (Michel-Beyerle, M.E., ed.), Springer-Verlag, Heidelberg, in the press 